\title{
Differential calculus on topological spaces with weak Markov structure I
}

\author{
Alexander Brudnyi
}

\section{Dedicated to the memory of Professor Mikael Passare.}

\begin{abstract}
The concept of a weak Markov set takes its origin from Whitney problems for differentiable functions on $\mathbb{R}^{n}$. In the present paper we study a version of the first Whitney problem of characterizing families of continuous functions satisfying certain differential relations on weak Markov sets. To this end we develop differential calculus on weak Markov sets similar to that on open subsets of $\mathbb{R}^{n}$. Then we show that some classical results for smooth functions and differential forms (such as Poincaré lemma, de Rham and Hartogs theorems, Künneth formulas, etc.) are valid also on certain weak Markov sets and more generally certain topological spaces with weak Markov structures. The class of such spaces includes, in particular, $C^{\infty}$ manifolds with boundaries and some Lipschitz and fractal topological manifolds. Thus the paper offers yet another approach to analysis on fractals, a developing area of modern mathematics that focuses on geometric and dynamical aspects of fractals.
\end{abstract}

\section{Introduction}

Motivated by boundary value problems for partial differential equations, classical trace and extension theorems characterize traces of spaces of generalized smoothness (e.g., Sobolev, Besov, etc.) to smooth submanifolds of a Euclidean space. But in many cases one needs similar results for subsets of a more complicated geometric structure (for instance, after the change of variables initial data may be situated on a Lipschitz surface). The subject is originated from the seminal 1934 Hassler Whitney papers [36] and [37], the first of which deals with the following problem: characterize collections of $m$-jets on a closed subset of $\mathbb{R}^{n}$ that are traces of $C^{m}$ jets, i.e., sets of Taylor polynomials of degree $m$ on $\mathbb{R}^{n}$ generated by $C^{m}$ functions. Whitney developed important analytic and geometric techniques which allowed

Mathematics Subject Classification (2010): Primary 26B05; Secondary 28A80, 18F15, 14F40. Keywords: Weak Markov set, Whitney problems, trace, extension, $C^{k}$ function, de Rham cohomology. 
him to solve this problem; moreover, he constructed a linear bounded extension operator acting from the trace space to $C^{m}\left(\mathbb{R}^{n}\right)$. In the second paper, marked by number I, he used his extension method to solve for univariate functions an essentially more difficult (known as the first Whitney) problem of characterizing continuous functions on an arbitrary closed subset of $\mathbb{R}^{n}$ that are traces of $C^{m}\left(\mathbb{R}^{n}\right)$ functions.

In accordance with the one-dimensional case, it was natural to formulate the second (Whitney) problem: does there exist a linear bounded extension operator from $C^{m}\left(\mathbb{R}^{n}\right)$ trace to a closed subset of $\mathbb{R}^{n}$ to $C^{m}\left(\mathbb{R}^{n}\right)$ ?

Although in the second half of the 20th century some important partial results were obtained, the problems remained unsolved almost 70 years. In the last decade a significant breakthrough in the area has been made by Charles Fefferman (2003-2009) (see, in particular, his survey [13] and references therein) who solved the second Whitney problem and made an important contribution towards the solution of the first Whitney problem justifying the, so-called, Yu. BrudnyiShvartsman finiteness principle for traces of $C^{m}$ functions to closed subsets of $\mathbb{R}^{n}$. Fefferman's results are based on multileveled, voluminous constructions that can be turned into algorithms for finite sets.

Whitney theory may be viewed as an important step towards differential calculus on closed subsets of Euclidean spaces. (For $C^{\infty}$ functions on subanalytic sets there are substantially more advanced theories developed by Len Bos and Pierre Milman [7] and by Edward Bierstone and Pierre Milman [5].) However, up to now it was not completely clear what property is responsible for a "differential calculus". It turns out that this is a "weak Markov property" introduced by the author and Yuri Brudnyi in [3] and shared by many classes of sets that arise in applications. For its definition, here and below, by $\mathcal{P}_{k, n}$ we denote the space of real polynomials on $\mathbb{R}^{n}$ of degree $k$ and by $Q_{r}(x) \subset \mathbb{R}^{n}$ the closed cube centered at $x$ of sidelength $2 r$.

Definition 1.1. A point $x$ of a subset $S \subset \mathbb{R}^{n}$ is said to be weak $k$-Markov if

$$
\varliminf_{r \rightarrow 0}\left\{\sup _{p \in \mathcal{P}_{k, n} \backslash\{0\}}\left(\frac{\sup _{Q_{r}(x)}|p|}{\sup _{S \cap Q_{r}(x)}|p|}\right)\right\}<\infty .
$$

A closed set $S \subset \mathbb{R}^{n}$ is said to be weak $k$-Markov if it contains a dense subset of weak $k$-Markov points.

The term weak Markov set is derived from that of Markov set introduced by Alf Jonsson and Hans Wallin in [22] by means of local Markov polynomial inequalities in connection with extension and trace problems for functions in Besov spaces, see Section 2 below for an equivalent definition. The class of weak $k$-Markov sets denoted by $\operatorname{Mar}_{k}^{*}\left(\mathbb{R}^{n}\right)$, contains, in particular, the closure of any open set, the Ahlfors $p$-regular compact subsets of $\mathbb{R}^{n}$ with $p>n-1$, a wide class of fractals of arbitrary positive Hausdorff measure and closures of unions of any combination of such sets. The class $\operatorname{Mar}_{0}^{*}\left(\mathbb{R}^{n}\right)$ (consisting of all nonempty closed subsets of $\mathbb{R}^{n}$ ) admits a filtration

$$
\operatorname{Mar}_{0}^{*}\left(\mathbb{R}^{n}\right) \supset \operatorname{Mar}_{1}^{*}\left(\mathbb{R}^{n}\right) \supset \operatorname{Mar}_{2}^{*}\left(\mathbb{R}^{n}\right) \supset \cdots \supset \operatorname{Mar}_{k}^{*}\left(\mathbb{R}^{n}\right) \supset \cdots .
$$


We set

$$
\operatorname{Mar}_{\infty}^{*}\left(\mathbb{R}^{n}\right):=\bigcap_{k \geq 0} \operatorname{Mar}_{k}^{*}\left(\mathbb{R}^{n}\right)
$$

and refer to elements of this class as weak $\infty$-Markov sets.

The purpose of the present paper is to study a version of the first Whitney problem of characterizing families of continuous functions satisfying certain differential relations on weak Markov sets. To this end we develop differential calculus on weak Markov sets first introducing (intrinsically) the notion of derivative of a function on such a set and then establishing its basic properties similar to those for derivatives of functions on $\mathbb{R}^{n}$. Next, we consider examples of problems for functions satisfying some differential relations and show, in particular, that many classical results for smooth functions and differential forms (such as Poincaré lemma, de Rham and Hartogs theorems, Künneth formulas, etc.) are valid also on certain weak Markov sets and more generally certain topological spaces with weak Markov structures. The class of such spaces includes, in particular, $C^{\infty}$ manifolds with boundaries and some Lipschitz and fractal topological manifolds. Thus the paper offers yet another approach to analysis on fractals, a developing area of modern mathematics that focuses on geometric and dynamical aspects of fractals. (For known approaches and major developments in the area, see, e.g., survey [33] and references therein.)

The paper is organized as follows. In Section 2 we formulate some basic properties of weak $k$-Markov sets accompanied by examples. In particular, we show that (a) unlike Markov sets, $\operatorname{Mar}_{k+1}^{*}\left(\mathbb{R}^{n}\right) \subsetneq \operatorname{Mar}_{k}^{*}\left(\mathbb{R}^{n}\right)$ for all $k \in \mathbb{Z}_{+}$; (b) direct products, closures of unions or of images under surjective $C^{1}$ maps of weak $k$ Markov sets are also weak $k$-Markov sets (in the corresponding Euclidean spaces); however, classes $\operatorname{Mar}_{k}^{*}\left(\mathbb{R}^{n}\right), n \geq 2$, are not invariant under bi-Lipschitz maps; and (c) for a fixed $k \geq 1$, each closed subset of $\mathbb{R}^{n}$ is disjoint union of a subset in $\operatorname{Mar}_{k}^{*}\left(\mathbb{R}^{n}\right) \cup\{\emptyset\}$ and a set of Hausdorff dimension at most $n-1$ whose closure does not contain subsets in $\operatorname{Mar}_{k}^{*}\left(\mathbb{R}^{n}\right)$.

In Section 3 we give the (intrinsic) definition of the derivative of a function $f: S \rightarrow \mathbb{R}$ at a weak 1-Markov point $x \in S \subset \mathbb{R}^{n}$ similar to the classical one: $f$ has derivative at $x$ if and only if there exists a vector $D_{S} f(x) \in \mathbb{R}^{n}$ such that

$$
f(y)=f(x)+\left\langle D_{S} f(x), y-x\right\rangle+o(\|y-x\|), \quad y \in S
$$

(here $\langle\cdot, \cdot\rangle$ and $\|\cdot\|$ are the standard inner product and the Euclidean norm on $\mathbb{R}^{n}$ ). Coordinates of $D_{S} f(x)$ are called partial derivatives of $f$ at $x$. We show that if a function $f$ has derivative at $x$, then it is restriction to $S$ of a differentiable at $x$ (in the usual sense) function $\tilde{f}: \mathbb{R}^{n} \rightarrow \mathbb{R}$ whose derivative at $x$ coincides with $D_{S} f(x)$ (see Proposition 3.2). Moreover, there are analogs of divided differences $\Delta_{x, i}^{k}$, $1 \leq k \leq n, i \in \mathbb{N}$, on $S$ with supports of cardinality $n+1$ converging to $x$ as $i \rightarrow \infty$ such that $D_{S} f(x)=\lim _{i \rightarrow \infty}\left(\Delta_{x, i}^{1}(f), \ldots, \Delta_{x, i}^{n}(f)\right)$ (see Proposition 3.3).

Similarly, one defines derivatives of order $m \leq k$ of a function $f: S \rightarrow \mathbb{R}$ at a weak $k$-Markov point $x \in S \subset \mathbb{R}^{n}$. If $S \in \operatorname{Mar}_{k}^{*}\left(\mathbb{R}^{n}\right)$ and $f \in C(S)$ has derivatives of order $m \leq k$ at all weak $k$-Markov points of $S$, then $m$-jets of $f$ consisting of 
all partial derivatives of order $\leq m$ of $f$ at these points are well defined. We say that such $f$ is $m$-times continuously differentiable (i.e., of class $C^{m}$ ), if the set of $m$-jets of $f$ satisfies, in each open relatively compact subset of $S$, the classical Whitney-Glaeser condition for some modulus of continuity $\omega$ (see Theorem 3.4). Then it follows that $f$ is restriction to $S$ of a $m$-times continuously differentiable function $\tilde{f}$ on $\mathbb{R}^{n}$.

We complete this section with formulations of the results related to the Whitney problems on weak Markov sets, that is, of finiteness principle and finite depth simultaneous extension for traces of differentiable functions to weak $k$-Markov sets, see Theorem 3.5 and Remark 3.6.

In Section 4, using intrinsic definitions of derivatives at weak $k$-Markov points of a set $S \in \operatorname{Mar}_{k}^{*}\left(\mathbb{R}^{n}\right)$, we define differential operators with continuous coefficients on $S$ of order $m \leq k$ whose domains consist of spaces of $m$-times continuously differentiable functions on $S$. Further, we present examples of solving certain homogeneous differential equations on $S$. In particular, we show that for some Cantor-type weak $k$-Markov sets traces of polynomials of degree $m \leq k-1$ to them are characterized as $(m+1)$-times continuously differentiable functions on $S$ whose partial derivatives of order $m$ are equal to zero (Theorem 4.3), and describe solutions of analogs of the Laplace equation (Theorem 4.5) and of the CauchyRiemann equations (Theorem 4.4) on such sets.

In Section 5 we introduce the class $\operatorname{Mar}_{k}^{*}$ of paracompact spaces with weak $k$-Markov structure, analogously to the definition of the class of manifolds with $C^{k}$ structure in differential topology, i.e., gluing together relatively open subsets of sets in $\operatorname{Mar}_{k}^{*}\left(\mathbb{R}^{n}\right)$ by means of local $C^{m}$ homeomorphisms. (In particular, every $C^{k}$ manifold and every subset of $\operatorname{Mar}_{k}^{*}\left(\mathbb{R}^{n}\right), n \in \mathbb{N}$, belong to $\operatorname{Mar}_{k}^{*}$.) We prove that any open cover of the space in $\operatorname{Mar}_{k}^{*}$ admits a $C^{k}$ partition of unity subordinate to it (Proposition 5.2), introduce notions of "tangent bundle", "differential" and " $C^{k}$ diffeomorphism" for spaces in $\operatorname{Mar}_{k}^{*}$ (generalizing similar notions for $C^{k}$ manifolds) and prove an analog of the Whitney embedding theorem for them (Proposition 5.4).

Next, we introduce the subclass $\operatorname{Mar}_{k, \Gamma}^{*} \subset \operatorname{Mar}_{k}^{*}$ (containing $C^{k}$ manifolds as well) consisting of spaces locally $C^{k}$ diffeomorphic to direct products of simple $\Gamma$ sets, that is, weak $k$-Markov sets situated between graphs (in $\mathbb{R}^{n+1}$ ) of continuous functions $f_{1}, f_{2}: \mathbb{K}^{n} \rightarrow \mathbb{R}, f_{1} \leq f_{2}$, where $\mathbb{K}^{n}:=(0,1)^{n} \subset \mathbb{R}^{n}$ is the open unit cube. (If, in this definition, $f_{1}=f_{2}$, then, in general, the graph of $f_{1}$ is a fractal in $\mathbb{R}^{n+1}$.) We show that fractal topological manifolds in $\operatorname{Mar}_{k, \Gamma}^{*}$ can be obtained by isotopic perturbations of $C^{1}$ manifolds (Theorem 5.5). Further, we introduce and study differential forms on spaces in $\operatorname{Mar}_{\infty, \Gamma}^{*}$. In particular, we prove that an analog of the classical Poincaré $d$-lemma for differential forms on $C^{\infty}$ manifolds is valid also for $d$-closed differential forms on spaces in $\operatorname{Mar}_{\infty, \Gamma}^{*}$. Thus the de Rham complex of differential forms on such a space is well defined and, as in the case of $C^{\infty}$ manifolds, the corresponding de Rham cohomology groups are isomorphic to the Čech cohomology groups of the space (Theorem 5.8). We also establish an analog of the Künneth formula for the de Rham cohomology groups of spaces in $\operatorname{Mar}_{\infty, \Gamma}^{*}$ (Theorem 5.13).

Sections 6-12 contain proofs of the main results of the paper. 
In a forthcoming paper, following ideas of H. Whitney [39], we introduce integration of differential forms over special "fractal" chains in spaces from $\operatorname{Mar}_{\infty, \Gamma}^{*}$ and establish an analog of the Stokes formula for them.

Acknowledgment. The paper arose in part out of discussions with Professor Vladimir Goldshtein during my visit to Ben Gurion University of the Negev in November 2010. I am grateful for his interest to the work. Also, I thank the anonymous referee for valuable remarks improving the presentation of the paper.

\section{Properties of weak Markov sets}

All properties except for (6), (10) and (11) proved in Section 6 below either follow directly from Definition 1.1 or have been established in [3] (see also Volume II, Chapter 10 in [2]).

(1) $\operatorname{Mar}_{0}^{*}\left(\mathbb{R}^{n}\right)$ consists of all nonempty closed sets in $\mathbb{R}^{n}$.

(2) $\operatorname{Mar}_{1}^{*}(\mathbb{R})$ consists of all infinite closed subsets of $\mathbb{R}$ without isolated points.

(3) If $S \in \operatorname{Mar}_{k}^{*}\left(\mathbb{R}^{n}\right)$ and $U \subset S$ is relatively open, then the closure $\bar{U} \in \operatorname{Mar}_{k}^{*}\left(\mathbb{R}^{n}\right)$.

(4) If $\left\{S_{i}\right\}_{i \in I} \subset \operatorname{Mar}_{k}^{*}\left(\mathbb{R}^{n}\right)$, then $\overline{\cup_{i \in I} S_{i}} \in \operatorname{Mar}_{k}^{*}\left(\mathbb{R}^{n}\right)$.

(5) For closed sets $S_{i} \subset \mathbb{R}^{n_{i}}, i=1,2$, their product $S_{1} \times S_{2} \in \operatorname{Mar}_{k}^{*}\left(\mathbb{R}^{n_{1}+n_{2}}\right)$ if and only if $S_{i} \in \operatorname{Mar}_{k}^{*}\left(\mathbb{R}^{n_{i}}\right), i=1,2$.

(6) Let $x$ be a weak $k$-Markov point of $S \subset \mathbb{R}^{n}$. Suppose a map $\varphi: \mathbb{R}^{n} \rightarrow \mathbb{R}^{m}$, $m \leq n$, is such that the derivative $D \varphi(x)$ of $\varphi$ at $x$ exists and has rank $m$. Then $\varphi(x)$ is a weak $k$-Markov point of $\varphi(S) \subset \mathbb{R}^{m}$. In particular, if the set of such points $x$ is dense in $S$, then $\overline{\varphi(S)} \in \operatorname{Mar}_{k}^{*}\left(\mathbb{R}^{m}\right)$.

(7) A closed set $S \subset \mathbb{R}^{n}$ belongs to the class of Markov sets, denoted by $\operatorname{Mar}\left(\mathbb{R}^{n}\right)$, if for some constant $c>0$ and every $x \in S, 0<r \leq \operatorname{diam} S$ and $p \in \mathcal{P}_{1, n}$,

$$
\sup _{Q_{r}(x)}|p| \leq c \cdot \sup _{S \cap Q_{r}(x)}|p| \text {. }
$$

If $S \in \operatorname{Mar}\left(\mathbb{R}^{n}\right)$, then the above condition holds for polynomials of every degree, see Chapter 2 in $[22]$. Hence, $\operatorname{Mar}\left(\mathbb{R}^{n}\right) \subset \operatorname{Mar}_{\infty}^{*}\left(\mathbb{R}^{n}\right)$.

A closed set $S \subset \mathbb{R}^{n}$ is called (Ahlfors) $d$-regular $(0 \leq d \leq n)$ if for every cube $Q_{r}(x)$ with $x \in S$ and $0<r<\operatorname{diam} S$

$$
c_{0} r^{d} \leq \mathcal{H}_{d}\left(S \cap Q_{r}(x)\right) \leq c_{1} r^{d}
$$

where $c_{0}, c_{1}>0$ are constants independent of $x$ and $r$ and $\mathcal{H}_{d}$ stands for the Hausdorff $d$-measure on $\mathbb{R}^{n}$.

Every $d$-regular compact subset of $\mathbb{R}^{n}$ with $d>n-1$ is Markov, see Chapter 2 of [22]. So the closure of unions of $d_{i}$-regular compact sets $S_{i} \subset \mathbb{R}^{n}$ with $d_{i}>n-1$, $i \in I$, is weak $\infty$-Markov. 
Example 2.1. The classical von Koch snowflake curve is $d$-regular with $d:=$ $\log 4 / \log 3$, see, e.g., [10] for its construction.

(8) Fix $k \in \mathbb{N}_{+} \cup\{\infty\}$. Every closed subset $S \subset \mathbb{R}^{n}$ is disjoint union of a (possibly empty) weak $k$-Markov subset and a set of Hausdorff dimension at most $n-1$.

In particular, let a closed set $S \subset \mathbb{R}^{n}$ have a base (in the relative topology) consisting of sets of Hausdorff dimension greater than $n-1$. Then $S$ is weak $\infty$-Markov. (For otherwise, nonempty open set $S \backslash S_{\max }$, where $S_{\max }$ is the maximal weak $\infty$-Markov subset of $S$, contains an element of the base with Hausdorff dimension greater than $n-1$.)

Using this fact one can prove, e.g., that the graph of the Weierstrass nowhere differentiable function

$$
\sum_{n=0}^{\infty} a^{n} \cos \left(b^{n} x\right), \quad \text { where } 0<a<1, b>1, a b>1,
$$

is weak $\infty$-Markov. (It follows from Theorem 4 in [32] and estimates in [15].)

More generally, it was proved in Theorem 5 of [32] that for a fixed $0<\alpha<1$ the graph of $\varphi(x)=\sum_{n=0}^{\infty} \beta^{-\alpha n} q\left(\beta^{n} x+\theta_{n}\right)$ with sufficiently large $\beta>1$ and arbitrary $\theta_{0}, \theta_{1}, \ldots$, where $q$ is a Lipschitz function on $\mathbb{R}$ of period one monotone and nonconstant on a compact interval, satisfies the above base topology assumption (for $n=2$ ). Hence, this graph belongs to $\operatorname{Mar}_{\infty}^{*}\left(\mathbb{R}^{2}\right)$.

(9) A point $x$ of a subset $S \subset \mathbb{R}^{n}$ is weak $k$-Markov if and only if there exist a convergent to 0 sequence of positive numbers $\left\{r_{i}\right\}_{i \in \mathbb{N}}$ and a sequence of finite subsets $F_{i} \subset S \cap Q_{r_{i}}(x)$ with card $F_{i}=\operatorname{dim} \mathcal{P}_{k, n}, i \in \mathbb{N}$, such that

$$
\sup _{i \in \mathbb{N}}\left\{\sup _{p \in \mathcal{P}_{k, n} \backslash\{0\}}\left(\frac{\sup _{Q_{r_{i}}(x)}|p|}{\sup _{F_{i}}|p|}\right)\right\}<\infty .
$$

The proof is a consequence of the following fact (cf. Proposition 3.5 in [3]):

Let $V$ be an $n$-dimensional vector space of bounded real functions on a set $X$ (in particular, card $X \geq n$ ). There exist a finite subset $F \subset X$ of $\operatorname{card} F=n$ and a constant $c=c(n)>0$ such that for every $v \in V$,

$$
\sup _{X}|v| \leq c \cdot \max _{F}|v| \text {. }
$$

(10) Unlike Markov sets, $\operatorname{Mar}_{k+1}^{*}\left(\mathbb{R}^{n}\right) \backslash \operatorname{Mar}_{k}^{*}\left(\mathbb{R}^{n}\right) \neq \emptyset$ for all $k \in \mathbb{Z}_{+}$.

In view of (5) it suffices to establish this property for $n=1$ only. It is done in Subsection 6.2 by constructing, for each $k \in \mathbb{Z}_{+}$, a Cantor-type subset of $\mathbb{R}$ of Hausdorff dimension zero belonging to $\operatorname{Mar}_{k}^{*}(\mathbb{R}) \backslash \operatorname{Mar}_{k+1}^{*}(\mathbb{R})$.

(11) Classes $\operatorname{Mar}_{k}^{*}(\mathbb{R}), k \in \mathbb{Z}_{+} \cup\{\infty\}$, are invariant under bi-Lipschitz maps (i.e., if $S \in \operatorname{Mar}_{k}^{*}(\mathbb{R})$ and $\varphi: S \rightarrow S^{\prime} \subset \mathbb{R}$ is a bi-Lipschitz map, then $\left.S^{\prime} \in \operatorname{Mar}_{k}^{*}(\mathbb{R})\right)$, while $\operatorname{Mar}_{k}^{*}\left(\mathbb{R}^{n}\right), n \geq 2, k \in \mathbb{N} \cup\{\infty\}$, are not.

To prove the second part of the statement for $k \in \mathbb{N}$ we construct a Lipschitz function $\varphi: \mathbb{R} \rightarrow \mathbb{R}$ whose graph $\Gamma_{\varphi} \in \operatorname{Mar}_{k}^{*}\left(\mathbb{R}^{2}\right) \backslash \operatorname{Mar}_{k+1}^{*}\left(\mathbb{R}^{2}\right)$ (see Subsection 6.3). 


\section{Traces of differentiable functions to weak Markov sets}

Results of this section are proved in Section 7.

Definition 3.1. A function $f: S \rightarrow \mathbb{R}$ is said to have derivatives of order $\leq m$ $(\leq k)$ at a weak $k$-Markov point $x \in S \subset \mathbb{R}^{n}$ if there exists a polynomial $T_{x}^{m}(\bar{f}) \in$ $\mathcal{P}_{m, n}$ such that

$$
\lim _{y \rightarrow x} \frac{\left|f(y)-T_{x}^{m}(f)(y)\right|}{\|y-x\|_{\infty}^{m}}=0 .
$$

If

$$
T_{x}^{m}(f)(z):=\sum_{|\alpha| \leq m} \frac{c_{\alpha}}{\alpha !}(z-x)^{\alpha}, \quad \alpha \in \mathbb{Z}_{+}^{n},|\alpha|:=\|\alpha\|_{1},
$$

then $c_{\alpha}$ is called the partial derivative of order $\alpha$ at $x$ and is denoted as $D_{S}^{\alpha} f(x)$.

(Here $\|\cdot\|_{\infty}$ and $\|\cdot\|_{1}$ stand for $\ell^{\infty}$-norm on $\mathbb{R}^{n}$ and $\ell^{1}$-norm on $\mathbb{Z}^{n}$, respectively.)

Proposition 3.2. A function $f: S \rightarrow \mathbb{R}$ has derivatives of order $\leq m(\leq k)$ at a weak $k$-Markov point $x \in S \subset \mathbb{R}^{n}$ if and only if there exists a function $\tilde{f}: \mathbb{R}^{n} \rightarrow \mathbb{R}$ having derivatives of order $\leq m$ at $x$ such that $\left.\tilde{f}\right|_{S}=f$. Moreover, the Taylor polynomial $T_{x}^{m}(\tilde{f})$ of degree $m$ at $x$ of any such extension $\tilde{f}$ coincides with $T_{x}^{m}(f)$.

Next, we express derivatives of $f: S \rightarrow \mathbb{R}$ in intrinsic terms using some analogs of divided differences.

Proposition 3.3. For a weak $k$-Markov point $x \in S \subset \mathbb{R}^{n}$, and a nonnegative integer number $m \leq k$ there exist a convergent to 0 sequence of positive numbers $\left\{r_{i}\right\}_{i \in \mathbb{N}}$, a sequence of finite subsets $F_{i}^{m} \subset S \cap Q_{r_{i}}(x) \backslash\{x\}$ with card $F_{i}=\operatorname{dim} \mathcal{P}_{m, n}$, a sequence of signed measures $\mu_{i}^{\alpha}, \alpha \in \mathbb{Z}_{+}^{n},|\alpha| \leq m$, on $F_{i}^{m}, i \in \mathbb{N}$, such that if a function $f: S \rightarrow \mathbb{R}$ has derivatives of order $m$ at $x$, then

$$
D_{S}^{\alpha} f(x)=\lim _{i \rightarrow \infty} \int_{F_{i}^{m}} f d \mu_{i}^{\alpha} .
$$

Recall that the space $C^{m, \omega}\left(\mathbb{R}^{n}\right)$ is defined by the norm

$$
\|f\|_{C^{m, \omega}\left(\mathbb{R}^{n}\right)}:=\sup _{\mathbb{R}^{n}}|f|+\max _{|\alpha|=m} \sup _{x \neq y} \frac{\left|D^{\alpha} f(x)-D^{\alpha} f(y)\right|}{\omega\left(\|x-y\|_{\infty}\right)}
$$

here $\omega:(0, \infty) \rightarrow(0, \infty)$ is a nondecreasing concave function with $\omega(0+)=0$. For a subset $S \subset \mathbb{R}^{n}$ by $C^{m, \omega}(S)$ we denote the trace space of continuous functions on $S$ equipped with the trace norm

$$
\|f\|_{C^{m, \omega}(S)}:=\inf \left\{\|g\|_{C^{m, \omega}\left(\mathbb{R}^{n}\right)} ;\left.g\right|_{S}=f\right\} .
$$

The following result characterizing functions in $C^{m, \omega}(S)$ is the analog of the classical Whitney-Glaeser theorem (see, e.g., Volume I, Section 2.2 in [2]).

Theorem 3.4. Let $S \subset \mathbb{R}^{n}$ be weak $k$-Markov, $k \in \mathbb{N}$. A function $f \in C(S)$ belongs to $C^{m, \omega}(S), m \leq k$, if and only if it has derivatives of order $\leq m(\leq k)$ at each weak $k$-Markov point $x \in S$ and there exists a constant $\lambda>0$ such that for all 
weak $k$-Markov points $x, y \in S, z \in\{x, y\}$,

$$
\begin{aligned}
& \max _{|\alpha| \leq m}\left|D_{S}^{\alpha} f(x)\right| \leq \lambda \quad \text { and } \\
& \max _{|\alpha| \leq m} \frac{\left|D^{\alpha}\left(T_{x}^{m}(f)-T_{y}^{m}(f)\right)(z)\right|}{\|x-y\|_{\infty}^{m-|\alpha|}} \leq \lambda \cdot \omega\left(\|x-y\|_{\infty}\right) .
\end{aligned}
$$

Moreover,

$$
\|f\|_{C^{m, \omega}(S)} \approx \inf \lambda
$$

with constants of equivalence depending only on $m$ and $n$.

Finally, we formulate a result proved in [3] that solves Whitney problems for the trace of space $C^{m, \omega}\left(\mathbb{R}^{n}\right)$ to weak $k$-Markov sets $(k \geq m)$.

Theorem 3.5. Let $S \subset \mathbb{R}^{n}$ be weak $k$-Markov, $k \in \mathbb{N}$, and $m \leq k$.

(a) Finiteness principle. Suppose that for $f \in C(S)$ its restriction to each subset $S^{\prime}$ of $S$ of cardinality at most $2\left(\begin{array}{c}n+m \\ n\end{array}\right)$ satisfies $\left\|\left.f\right|_{S^{\prime}}\right\|_{C^{m, \omega}\left(S^{\prime}\right)} \leq 1$. Then $f \in C^{m, \omega}(S)$ and there exists $c=c(m, n)>0$ such that $\|f\|_{C^{m, \omega}(S)} \leq c$.

(b) Finite depth simultaneous extension. There exists a linear bounded extension operator $E: C^{m, \omega}(S) \rightarrow C^{m, \omega}\left(\mathbb{R}^{n}\right)$,

$$
(E f)(x):=\left\{\begin{aligned}
\sum_{i=1}^{\infty} \lambda_{i}(x) f\left(x_{i}\right) & \text { if } x \in \mathbb{R}^{n} \backslash S, \\
f(x) & \text { if } x \in S ;
\end{aligned}\right.
$$

here all $\lambda_{i} \in C^{\infty}\left(\mathbb{R}^{n}\right)$ and have compact supports in $\mathbb{R}^{n} \backslash S$, all $x_{i} \in S$ and for each $x \in \mathbb{R}^{n} \backslash S$ the number of nonzero terms in the above sum is at most $\left(\begin{array}{c}n+m \\ n\end{array}\right) \cdot w$, where $w$ is the order of the Whitney cover of $\mathbb{R}^{n} \backslash S$.

Remark 3.6. If $S \subset \mathbb{R}^{n}$ is an arbitrary closed set, then, as it was discovered by Fefferman [11], a statement similar to (a) is valid with the finiteness constant bounded by $\left(1+\operatorname{dim} \mathcal{P}_{m, n}\right)^{3 \cdot \operatorname{dim} \mathcal{P}_{m, n}}$. Later this bound was replaced by $2^{\operatorname{dim} \mathcal{P}_{m, n}}$ due to Bierstone-Milman [6] and, independently and by a different method, Shvartsman [34]. A linear bounded extension operator $C^{m, \omega}(S) \rightarrow C^{m, \omega}\left(\mathbb{R}^{n}\right)$ in this case was constructed by Fefferman [12]. Using a modification of his method Luli [26] constructed an extension operator $C^{m, \omega}(S) \rightarrow C^{m, \omega}\left(\mathbb{R}^{n}\right)$ of finite depth, cf. (b).

\section{Examples of extension problems}

Definition 4.1. A function $f$ on an open subset $U$ of a weak $k$-Markov set $S \subset \mathbb{R}^{n}$ is said to belong to the space $C^{m}(U), m \leq k$, if for each $x \in U$ there is its open neighbourhood $O_{x} \subset U$ such that $\left.f\right|_{O_{x}}$ satisfies conditions of Theorem 3.4 with a modulus of continuity $\omega_{x}$. Here $C^{\infty}(U):=\cap_{\ell=1}^{\infty} C^{\ell}(U)$.

We define $C^{m}\left(U, \mathbb{R}^{p}\right)$ as the space of maps $U \rightarrow \mathbb{R}^{p}$ with coordinates in $C^{m}(U)$. 
Using Theorem 3.4 and a smooth partition of unity subordinate to a suitable cover of $U$ by open subsets of $\mathbb{R}^{n}$, one easily shows that $f \in C^{m}(U)$ iff it is restriction to $U$ of a $C^{m}$ function defined in an open neighbourhood of $U$ in $\mathbb{R}^{n}$. In the case $m=\infty$ this follows from a result of Hestens [18].

Let $S \in \operatorname{Mar}_{k}^{*}\left(\mathbb{R}^{n}\right)$ and $S_{0} \subset S$ be a dense subset of weak $k$-Markov points of $S$. For each $f \in C^{m}(S), m \leq k$, the function $D_{S}^{\alpha} f \in C(S),|\alpha| \leq m$, is defined as the extension by continuity to $S$ of the function $D_{S}^{\alpha}\left(\left.f\right|_{S_{0}}\right)$ (which is locally uniformly continuous on $S_{0}$ due to Theorem 3.4). Next, for a differential operator $P_{D}:=\sum_{|\alpha| \leq m} a_{\alpha} D^{\alpha}, a_{\alpha} \in C(W)$, where $W \subset \mathbb{R}^{n}$ is an open neighbourhood of $S$, we define

$$
P_{D_{S}} f:=\sum_{|\alpha| \leq m}\left(\left.a_{\alpha}\right|_{S}\right) D_{S}^{\alpha} f .
$$

If $f$ is restriction to $S$ of a $C^{m}$ function $\hat{f}$ defined in an open neighbourhood of $S$ in $W$, then (see Proposition 3.2)

$$
P_{D_{S}} f=\left.\left(P_{D} \hat{f}\right)\right|_{S}
$$

In what follows for a subset $O$ of a Hausdorff topological space $X, \partial O:=\bar{O} \backslash O^{\circ}$ and $O^{c}:=X \backslash O$ stand for its boundary and complement in $X$.

In the formulated examples we deal with Cantor-type sets of the form

$$
S:=\bar{\Omega} \backslash\left(\bigcup_{j \geq 1} D_{j}\right),
$$

where $\Omega$ is a bounded open subset of $\mathbb{R}^{n}$ or $\mathbb{C}^{n}$ and $\left\{D_{j}\right\}_{j \geq 1}$ is a family of mutually disjoint domains in $\Omega$ such that $\partial D_{j}=\partial\left(\bar{D}_{j}\right)^{c}$ for all $j$.

Example 4.2. The Sierpiński gasket is a $d$-regular set, $d:=\log 3 / \log 2$, of the form (4.3) obtained by repeatedly removing open equilateral triangles from an initial equilateral triangle, see, e.g., [10].

Our first example describes traces of polynomials to a class of Cantor-type weak Markov sets.

Theorem 4.3. Let $S \subset \mathbb{R}^{n}, n \geq 2$, be a weak $k$-Markov set, $k \geq 2$, of the form (4.3) such that $\Omega \Subset \mathbb{R}^{n}$ and all $\left(\bar{D}_{j}\right)^{c}$ are domains. Suppose $f \in C^{m+1}(S)$, $m \leq k-1$, satisfies $D_{S}^{\alpha} f=0$ for all $|\alpha|=m$. Then there exists a unique polynomial $p_{f} \in \mathcal{P}_{m-1, n}$ such that $\left.p_{f}\right|_{S}=f$. In particular, $S$ is connected.

Next we present a Hartogs-type theorem describing traces of holomorphic functions to a class of Cantor-type weak Markov sets.

Theorem 4.4. Let $S \subset \mathbb{C}^{n}, n \geq 2$, be a weak $k$-Markov set, $k \geq 2$, of the form (4.3) such that $\Omega \Subset \mathbb{C}^{n}$ and all $\left(\bar{D}_{j}\right)^{c}$ are domains. Let $f \in C_{\mathbb{C}}^{2}(S):=$ $C^{2}(S) \otimes \mathbb{C}$ satisfy $\bar{\partial}_{S} f=0$. Then there exists a unique holomorphic function $F \in \mathcal{O}(\Omega) \cap C_{\mathbb{C}}^{1}(\bar{\Omega})$ such that $\left.F\right|_{S}=f$ and $\sup _{\bar{\Omega}}|F|=\sup _{S}|f|$. 
Our third example describes solutions of Laplace-type equations on a class of Cantor-type weak Markov sets. In its formulation $C^{2, \alpha}\left(\mathbb{R}^{n}\right)$ stands for the space of bounded $C^{2}$ functions on $\mathbb{R}^{n}$ whose second-order derivatives satisfy the Hölder condition with exponent $\alpha$.

Theorem 4.5. Let $S \subset \mathbb{R}^{n}, n \geq 3$, be a weak $k$-Markov set, $k \geq 2$, of the form (4.3). Let $f \in C^{2, \alpha}(S), 0<\alpha<1$, satisfy $\Delta_{S} f=0$. Then there exist functions $F_{1}, F_{2} \in C^{2, \alpha}\left(\mathbb{R}^{n}\right)$ harmonic in $\cup_{j \geq 1} D_{j}$ and $\left(\overline{\cup_{j \geq 1} D_{j}}\right)^{c}$, respectively, such that $\lim _{x \rightarrow \infty} F_{i}(x)=0, i=1,2$, and $\left.\left(F_{1}+F_{2}\right)\right|_{S}=f$. Moreover, if $F_{1}^{\prime}, F_{2}^{\prime} \in$ $C^{2, \alpha}\left(\mathbb{R}^{n}\right)$ is any other pair of such functions, then $F_{1}^{\prime}=F_{1}$ on $\cup_{j \geq 1} D_{j}$ and $F_{2}^{\prime}=F_{2}$ on $\left(\overline{\cup_{j \geq 1} D_{j}}\right)^{c}$.

\section{Spaces with weak Markov structure}

\subsection{Basic definitions and properties}

Most definitions of this subsection are similar to those of the theory of standard $C^{k}$ manifolds, see, e.g., [30].

Definition 5.1. A weak $k$-Markov structure on a second countable Hausdorff space $S$ is a collection of pairs $\mathcal{D}:=\left\{\left(U_{\alpha}, \varphi_{\alpha}\right)\right\}_{\alpha \in \Lambda}$, called charts, such that

(1) $\left(U_{\alpha}\right)_{\alpha \in \Lambda}$ is an open cover of $S$;

(2) Each $\varphi_{\alpha}$ is a homeomorphism of $U_{\alpha}$ onto a relatively open subset of a set in $\operatorname{Mar}_{k}^{*}\left(\mathbb{R}^{n}\right)$ such that for all $\alpha, \beta \in \Lambda$ maps $\varphi_{\beta} \circ \varphi_{\alpha}^{-1}: \varphi_{\alpha}\left(U_{\alpha} \cap U_{\beta}\right) \rightarrow$ $\varphi_{\beta}\left(U_{\alpha} \cap U_{\beta}\right)\left(\subset \mathbb{R}^{n}\right)$ are of class $C^{k}$;

(3) $\mathcal{D}$ is maximal with respect to property (2).

A function $f$ on an open subset $U \subset S$ is of class $C^{k}$ if $f \circ \varphi_{\alpha} \in C^{k}\left(\varphi_{\alpha}\left(U \cap U_{\alpha}\right)\right)$ for all $\left(U_{\alpha}, \varphi_{\alpha}\right) \in \mathcal{D}$.

The definition implies that $S$ is paracompact and of covering dimension at most $n$. Moreover, it has a base of topology consisting of relatively compact open subsets.

The class of spaces with weak $k$-Markov structure will be denoted $\operatorname{Mar}_{k}^{*}$. If $M$ is a $C^{k}$ manifold, then by $\operatorname{Mar}_{k}^{*}(M) \subset \operatorname{Mar}_{k}^{*}$ we denote the family of all weak $k$-Markov closed subsets $S \subset M$, i.e., such that in Definition 5.1 each $\left(U_{\alpha}, \varphi_{\alpha}\right)$ has the form $U_{\alpha}:=V_{\alpha} \cap S$ and $\varphi_{\alpha}:=\left.\psi_{\alpha}\right|_{U_{\alpha}}$, where $\left(V_{\alpha}, \psi_{\alpha}\right)$ is a $C^{k}$ chart on $M$. In particular, every $C^{k}$ manifold and weak $k$-Markov subset of $\mathbb{R}^{n}$ belong to Mar $_{k}^{*}$.

Proposition 5.2. Let $S \in \operatorname{Mar}_{k}^{*}$. For any open cover of $S$ there exists a $C^{k}$ partition of unity subordinate to it.

For $S_{1}, S_{2} \in \operatorname{Mar}_{k}^{*}$ a continuous map $f: S_{1} \rightarrow S_{2}$ is said to be of class $C^{k}$ if it is of class $C^{k}$ in all suitable charts $\left(U_{\alpha 1}, \varphi_{\alpha 1}\right) \in \mathcal{D}_{1}$ and $\left(U_{\alpha 2}, \varphi_{\alpha 2}\right) \in \mathcal{D}_{2}$ on $S_{1}$ and $S_{2}$, respectively (i.e., $\left.\varphi_{\alpha 2} \circ f \circ \varphi_{\beta 1}^{-1} \in C^{k}\left(\left(\varphi_{\beta 1} \circ f^{-1}\right)\left(U_{\alpha 2}\right), \varphi_{\alpha 2}\left(U_{\alpha 2}\right)\right)\right)$. 
Every $C^{k}$ map $f: S_{1} \rightarrow S_{2}$ determines an injective morphism of algebras $f^{*}: C^{k}(U) \rightarrow C^{k}\left(f^{-1}(U)\right), U \subset S_{2}$ is open (here $f^{*}$ is the pullback by $f$ ).

For $S \subset \operatorname{Mar}_{k}^{*}\left(\mathbb{R}^{n}\right), k \geq 1$, the tangent bundle $T S \rightarrow S$ on $S$ is defined as the restriction to $S$ of the tangent bundle $T \mathbb{R}^{n} \rightarrow \mathbb{R}^{n}$ on $\mathbb{R}^{n}$. Clearly, $T S \in \operatorname{Mar}_{k}\left(T \mathbb{R}^{n}\right)$. Now, for a $C^{k}$ map $f: S \rightarrow \mathbb{R}^{p}$ the differential $F f: T S \rightarrow T \mathbb{R}^{p}$ is defined as the restriction of the differential $D \hat{f}: T U \rightarrow T \mathbb{R}^{p}$, where $\hat{f}$ is a $C^{k}$ extension of $f$ to an open neighbourhood $U \subset \mathbb{R}^{n}$ of $S$. According to Propositions 3.2, 3.3, the definition of $D f$ does not depend on the choice of the $C^{k}$ extension $\hat{f}$ and, in intrinsic terms, $D f:=\left(f,\left(D_{S}^{j} f_{i}\right)_{1 \leq i \leq n, 1 \leq j \leq p}\right)$, where $f:=\left(f_{1}, \ldots, f_{p}\right)$.

Similarly, if $S \in \operatorname{Mar}_{k}^{*}$, then the tangent bundle $T S \rightarrow S$ is defined as the quotient space of disjoint union $\bigsqcup_{\alpha \in \Lambda} T U_{\alpha}$, where $T U_{\alpha} \rightarrow U_{\alpha}$ is the pullback by $\varphi_{\alpha}$ of the bundle $T \overline{\varphi_{\alpha}\left(U_{\alpha}\right)}$, by means of the following equivalence relation:

$$
T U_{\alpha} \ni v \sim w \in T U_{\beta} \Longleftrightarrow\left(\varphi_{\beta}\right)_{*}(w)=D\left(\varphi_{\beta} \circ \varphi_{\alpha}^{-1}\right)\left(\left(\varphi_{\alpha}\right)_{*}(v)\right) ;
$$

here $\left(U_{\alpha}, \varphi_{\alpha}\right) \in \mathcal{D}$ and $\left(\varphi_{\alpha}\right)_{*}:\left.T U_{\alpha} \rightarrow T \overline{\varphi_{\alpha}\left(U_{\alpha}\right)}\right|_{\varphi_{\alpha}\left(U_{\alpha}\right)}$ is the isomorphism of bundles generated by $\varphi_{\alpha}$. The weak $k$-Markov structure on $T S$ is given by the collection of charts $\left\{\left(T U_{\alpha},\left(\varphi_{\alpha}\right)_{*}\right)\right\}_{\alpha \in \Lambda}$.

Next, if $f: S_{1} \rightarrow S_{2}$ is a $C^{k}$ map between $S_{1}, S_{2} \in \operatorname{Mar}_{k}^{*}$, then the differential is a morphism of bundles $F f: T S_{1} \rightarrow T S_{2}$ defined in charts $\left(U_{\alpha 1}, \varphi_{\alpha 1}\right) \in \mathcal{D}_{1}$ and $\left(U_{\beta 2}, \varphi_{\alpha 2}\right) \in \mathcal{D}_{2}$ such that $f\left(U_{\alpha 1}\right) \subset U_{\beta 2}$ by the formula

$$
D f(v):=\left(\varphi_{\beta 2}\right)_{*}^{-1}\left(D\left(\varphi_{\beta 2} \circ f \circ \varphi_{\alpha 1}^{-1}\right)\left(\left(\varphi_{\alpha 1}\right)_{*}(v)\right)\right) \text {. }
$$

We say that $f: S_{1} \rightarrow S_{2}$ is a $C^{k}$ embedding if $f$ is an injection and $D f$ is injective on each fibre of $T S_{1}$. If, in addition, $f$ is a homeomorphism, then it is called a $C^{k}$ diffeomorphism.

Proposition 5.3. Suppose $S_{1}, S_{2} \in \operatorname{Mar}_{k}^{*}, k \geq 1$, and $f: S_{1} \rightarrow S_{2}$ is a $C^{k}$ diffeomorphism. Then $f^{-1}: S_{2} \rightarrow S_{1}$ is a $C^{k}$ diffeomorphism as well.

Let $S \subset \mathbb{R}^{p}$ be a closed subset and $S \in \operatorname{Mar}_{k}^{*}$. We say that the weak $k$-Markov structure $\mathcal{D}$ on $S$ is induced from $\mathbb{R}^{p}$ if each $\varphi_{\alpha}$ in Definition 5.1 for $S$ is restriction of a $C^{k}$ map into $\mathbb{R}^{n}$ defined in an open neighbourhood of $U_{\alpha}$ in $\mathbb{R}^{p}$.

Our next result is an analog of the Whitney embedding theorem [38].

Proposition 5.4. Suppose $S \in \operatorname{Mar}_{k}^{*}, k \geq 1$, and the rank of TS is $n$. There exists a proper $C^{k}$ embedding $f: S \rightarrow \mathbb{R}^{N}$, where $N:=2 n+1$ if $k \geq 2$, such that $f(S) \in \operatorname{Mar}_{k}^{*}$ and has weak $k$-Markov structure induced from $\mathbb{R}^{N}$. Moreover, $f: S \rightarrow f(S)$ is a $C^{k}$ diffeomorphism.

(In case $S \in \operatorname{Mar}_{k}^{*}(M), k \geq 2$, where $\operatorname{dim} M=n$, one can replace $2 n+1$ by $2 n$ applying the strong Whitney embedding theorem to $M$.)

\subsection{Calculus of differential forms}

In what follows $\mathbb{K}^{n}:=(0,1)^{n} \subset \mathbb{R}^{n}$ stands for the open unit cube.

Let $f_{i j}: \mathbb{K}^{n_{i}} \rightarrow \mathbb{R}, 1 \leq i \leq \ell, j=1,2$, be continuous functions such that $f_{i 1} \leq$ $f_{i 2}$ and closures of sets $\Gamma_{f_{i 1}, f_{i 2}}^{n_{i}}:=\left\{\left(x, t f_{i 1}(x)+(1-t) f_{i 2}(x)\right) ; x \in \mathbb{K}^{n_{i}}, t \in[0,1]\right\}$ 
are weak $k$-Markov subsets of $\mathbb{R}^{n_{i}+1}$ for all $1 \leq i \leq m$. A set of the form $\Gamma_{f_{11}, f_{12}}^{n_{1}} \times$ $\cdots \times \Gamma_{f_{\ell 1}, f_{\ell 2}}^{n_{\ell}} \subset \mathbb{R}^{n_{1}+1} \times \cdots \times \mathbb{R}^{n_{\ell}+1}\left(=: \mathbb{R}^{n}, n:=\sum_{i=1}^{\ell}\left(n_{i}+1\right)\right)$ will be called a $\Gamma$ set. Each component $\Gamma_{f_{i 1}, f_{i 2}}^{n_{i}} \subset \mathbb{R}^{n_{i}+1}$ will be called a simple $\Gamma$ set.

By $\operatorname{Mar}_{k, \Gamma}^{*} \subset \operatorname{Mar}_{k}^{*}$ we denote the subclass of sets $S$ such that for each $x \in S$ there exists a chart $\left(U_{\alpha}, \varphi_{\alpha}\right)$ with $x \in U_{\alpha}$ and $\varphi_{\alpha}\left(U_{\alpha}\right) \subset \mathbb{R}^{n}$ a $\Gamma$ set.

If $M$ is a $C^{k}$ manifold, then by $\operatorname{Mar}_{k, \Gamma}^{*}(M) \subset \operatorname{Mar}_{k, \Gamma}^{*}$ we denote the family of closed subsets $S \subset M$ such that in the above definition each $\left(U_{\alpha}, \varphi_{\alpha}\right)$ has the form $U_{\alpha}:=V_{\alpha} \cap S$ and $\varphi_{\alpha}:=\left.\psi_{\alpha}\right|_{U_{\alpha}}$, where $\left(V_{\alpha}, \psi_{\alpha}\right)$ is a $C^{k}$ chart on $M$.

In particular, every $C^{k}$ manifold with a boundary, $k \geq 1$, belongs to $\operatorname{Mar}_{k, \Gamma}^{*}$. However, one can show that the von Koch snowflake curve with the weak $\infty$-Markov structure induced from $\mathbb{R}^{2}$ does not belong to $\operatorname{Mar}_{\infty, \Gamma}^{*}$. The next result shows that this class contains sufficiently many fractal topological manifolds.

Theorem 5.5. Let $M$ be a $C^{k}$ submanifold, $k \geq 1$, of an $n$-dimensional $C^{\infty}$ Riemannian manifold $X$ of dimension $m \geq\lfloor n / 2\rfloor \geq 1$ and let $U \subset X$ be an open neighbourhood of $M$. There is an open neighbourhood $N \subset U$ of $M$ such that

(A) For each $d \in[m, n]$ there exist a topological submanifold $M_{d} \subset \operatorname{Mar}_{\infty, \Gamma}^{*}(N)$ having a base of topology of sets of Hausdorff dimension $d$ and an isotopy $h_{d, t}$ : $M_{d} \rightarrow N, t \in[0,1]$, such that $h_{d, 0}=\mathrm{id}, h_{d, 1}\left(M_{d}\right)=M$, each $h_{d, t}\left(M_{d}\right) \in$ $\operatorname{Mar}_{\infty, \Gamma}^{*}(N), 0 \leq t<1$, and $h_{d, t}: M_{d} \rightarrow h_{d, t}\left(M_{d}\right)$ is a $C^{k}$ diffeomorphism for all such $t$. Moreover, $M_{m}$ is a Lipschitz manifold and $h_{m, 1}: M_{m} \rightarrow M$ is locally bi-Lipschitz.

(B) For each $l \in \mathbb{N}$ there exist a Lipschitz submanifold $M_{l} \in \operatorname{Mar}_{l, \Gamma}^{*}(N) \backslash$ $\operatorname{Mar}_{l+1, \Gamma}^{*}(N)$ and an isotopy $h_{l, t}: M_{l} \rightarrow N, t \in[0,1]$, such that $h_{l, 0}=\mathrm{id}, h_{l, 1}\left(M_{l}\right)$ $=M$, each $h_{l, t}\left(M_{l}\right) \in \operatorname{Mar}_{l, \Gamma}^{*}(U) \backslash \operatorname{Mar}_{l+1, \Gamma}^{*}(N), 0 \leq t<1$ and $h_{l, t}: M_{l} \rightarrow h_{l, t}\left(M_{l}\right)$, is a $C^{p}$ diffeomorphism, $p:=\min \{l, k\}$, for all such $t$. Moreover, $h_{l, 1}: M_{l} \rightarrow M$ is locally bi-Lipschitz.

For $S_{1}, S_{2} \in \operatorname{Mar}_{k}^{*}, k \geq 1$, a continuous map $f: S_{1} \rightarrow S_{2}$ is said to be locally Lipschitz if it is locally Lipschitz in all suitable charts $\left(U_{\alpha 1}, \varphi_{\alpha 1}\right) \in \mathcal{D}_{1}$ and $\left(U_{\alpha 2}, \varphi_{\alpha 2}\right) \in \mathcal{D}_{2}$ on $S_{1}$ and $S_{2}$, respectively (that is, $\varphi_{\alpha 2} \circ f \circ \varphi_{\beta 1}^{-1} \in \operatorname{Lip}_{\text {loc }}\left(\left(\varphi_{\beta 1} \circ\right.\right.$ $\left.\left.f^{-1}\right)\left(U_{\alpha 2}\right), \varphi_{\alpha 2}\left(U_{\alpha 2}\right)\right)$, where the metric structures of sets in the brackets are induced from those of the corresponding Euclidean spaces). Clearly, the definition does not depend on the choice of charts and each $C^{k}$ map $S_{1} \rightarrow S_{2}$ is locally Lipschitz as well. We say that $f: S_{1} \rightarrow S_{2}$ is locally bi-Lipschitz if $f$ is a homeomorphism and $f, f^{-1}$ are locally Lipschitz maps. Since the class of locally bi-Lipschitz homeomorphisms preserves Hausdorff dimension and each $m$-dimensional $C^{k}$ manifold $M, m \geq 1, k \geq 1$, admits a proper $C^{k}$ embedding into $\mathbb{R}^{2 m}$, Theorem 5.5 shows that there is a family of the cardinality of the continuum of locally bi-Lipschitz nonequivalent weak $\infty$-Markov structures on $M$.

For $S \in \operatorname{Mar}_{k}^{*}, k \geq 1$, the cotangent bundle $T^{*} S \rightarrow S$ is a bundle dual to the tangent bundle $T S \rightarrow S$ and equipped with a weak $k$-Markov structure induced by that on TS. A differential form $\omega$ of class $C^{k}$ on $S$ of degree $\operatorname{deg} \omega=p$ is a $C^{k}$ section of the bundle $\wedge^{p} T^{*} S$. If rank of $T S$ is $n$ and $x=\left(x_{1}, \ldots, x_{n}\right)$ are 
coordinates in a chart on $S$, then $\omega$ can be written locally in these coordinates as

$$
\omega:=\sum_{|I|=p} c_{I}(x) d x_{I},
$$

where $c_{I}$ are of class $C^{k}$ and for $I=\left(i_{1}, \ldots, i_{s}\right), 1 \leq i_{\ell} \leq n, 1 \leq \ell \leq s$, we define $|I|:=s$ and $d x_{I}:=d x_{i_{1}} \wedge d x_{i_{2}} \wedge \cdots \wedge d x_{i_{s}}$; here $d x_{1}, \ldots, d x_{n}$ are local coordinates on $T^{*} S$ in the chart, dual to the coordinates $\partial / \partial x_{1}, \ldots, \partial / \partial x_{n}$ on $T S$ there (pulled back from $T \mathbb{R}^{n}$ by the map defining this chart).

In what follows we assume that $S \in \operatorname{Mar}_{\infty}^{*}$. By $\Omega^{p}(U)$ we denote the vector space of $C^{\infty}$ differential forms of degree $p$ on an open set $U \subset S$. The differential $d: \Omega^{p}(U) \rightarrow \Omega^{p+1}(U)$ is defined exactly as in the case of differential forms on $C^{\infty}$ manifolds (e.g., if $S \in \operatorname{Mar}_{\infty}^{*}\left(\mathbb{R}^{n}\right)$ and $\omega=\sum_{|I|=p} c_{I} d x_{I} \in \Omega^{p}(S)$, where $x_{1}, \ldots, x_{n}$ are coordinates on $\mathbb{R}^{n}$, then $\left.d \omega:=\sum_{|I|=p}\left(\sum_{i=1}^{n} D_{S}^{i} c_{I} d x_{i}\right) \wedge d x_{I}\right)$.

The following result generalizes the classical Poincaré $d$-lemma for differential forms on $C^{\infty}$ manifolds.

Theorem 5.6. If $S \in \operatorname{Mar}_{\infty, \Gamma}^{*}$ and $\omega \in \Omega^{p}(S)$ is $d$-closed, i.e., $d \omega=0$, then for each $x \in S$ there exists an open neighbourhood $U_{x}$ of $x$ and, for $p \geq 1$, a form $\eta_{x} \in \Omega^{p-1}\left(U_{x}\right)$ such that $d \eta_{x}=\omega$. If $p=0$, then $\omega$ is locally constant.

By $\Omega_{S}^{p}$ we denote the sheaf of germs of $C^{\infty}$ differential forms of degree $p$ on $S \in \operatorname{Mar}_{\infty}^{*}$. Then from Proposition 5.2 we get (see, e.g., [19] for basic definitions and results of algebraic topology used below):

Proposition 5.7. $\Omega_{S}^{p}$ is a fine sheaf.

Combining Theorem 5.6 with Proposition 5.7 we obtain straightforwardly:

Theorem 5.8. Let $S \in \operatorname{Mar}_{\infty, \Gamma}^{*}$ and $\operatorname{rank} T S=n$. Then we have the following resolution of the constant sheaf $\mathbb{R}$ on $S$ by the sheaves of differential forms:

$$
0 \longrightarrow \mathbb{R} \stackrel{d}{\longrightarrow} \Omega_{S}^{0} \stackrel{d}{\longrightarrow} \Omega_{S}^{1} \stackrel{d}{\longrightarrow} \cdots \stackrel{d}{\longrightarrow} \Omega_{S}^{n} \stackrel{d}{\longrightarrow} 0 .
$$

In particular, $\check{H}^{i}(S, \mathbb{R})=H_{d R}^{i}(S, \mathbb{R})$, where $\check{H}^{i}(S, \mathbb{R})$ is the $\check{C}$ ech cohomology of sheaf $\mathbb{R}$ on $S$ and

$$
H_{d R}^{i}(S, \mathbb{R}):=\frac{\operatorname{Ker}\left(\Omega^{i}(S) \stackrel{d}{\longrightarrow} \Omega^{i+1}(S)\right)}{d\left(\Omega^{i-1}(S)\right)}
$$

Since $\check{H}^{i}(S, \mathbb{R})=0$ for $i>\operatorname{dim}_{c} S$, where $\operatorname{dim}_{c}$ stands for the covering dimension, Theorem 5.8 yields

Corollary 5.9. For $i>\operatorname{dim}_{c} S$ each d-closed form in $\Omega^{i}(S)$ is d-exact.

The statement of Theorem 5.8 is not valid if $S \notin \operatorname{Mar}_{\infty, \Gamma}^{*}$ as the following simple example shows. 
Example 5.10. Let $S \subset \operatorname{Mar}_{\infty}^{*}(\mathbb{R})$ be the standard Cantor set in $[0,1]$. Then $\check{H}^{0}(S, \mathbb{R})$ consists of continuous locally constant functions on $S$. In particular, each such function assigns finitely many values. Let $[0,1] \backslash S:=\cup_{j=1}^{\infty}\left(a_{j}, b_{j}\right)$ and $\varphi$ be a positive $C^{\infty}$ function on $(0,1)$ having zeros of infinite order at 0 and 1 . For a uniformly bounded sequence $\left\{c_{j}\right\} \subset \mathbb{R}_{+}$consider a sequence of positive functions $\varphi_{j} \in C^{\infty}\left(\left(a_{j}, b_{j}\right)\right), \varphi_{j}(x):=c_{j} e^{-\left(b_{j}-a_{j}\right)} \varphi\left(\frac{x-a_{j}}{b_{j}-a_{j}}\right), j \in \mathbb{N}$. Then the function $\tilde{\varphi}$ equals $\varphi_{j}$ on $\left(a_{j}, b_{j}\right), j \in \mathbb{N}$, and 0 on $S \cup(\mathbb{R} \backslash[0,1])$ is $C^{\infty}$ on $\mathbb{R}$. We set $\psi(x):=$ $\int_{-\infty}^{x} \tilde{\varphi}(t) d t, x \in \mathbb{R}$. By the definition $\psi \in C^{\infty}(\mathbb{R})$ and $d\left(\left.\psi\right|_{S}\right)=0$. However, the range of $\left.\psi\right|_{S}$ is not finite. This shows that $\check{H}^{0}(S, \mathbb{R})$ is a proper subset of $H_{d R}^{0}(S, \mathbb{R})$.

In general, for $S \in \operatorname{Mar}_{\infty}^{*}$ as in the case of $C^{\infty}$ manifolds (using Proposition 5.7) we obtain canonical homomorphisms of groups $h_{S}^{i}: \check{H}^{i}(S, \mathbb{R}) \rightarrow H_{d R}^{i}(S, \mathbb{R})$ such that if $f: S_{1} \rightarrow S_{2}$ is a $C^{\infty}$ map of spaces in Mar $_{\infty}^{*}$, then for all $i$ the following diagrams:

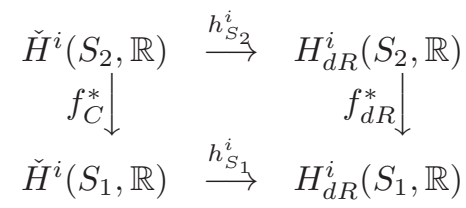

are commutative. Here $f_{C}^{*}$ and $f_{d R}^{*}$ are homomorphisms induced by $f$.

As another consequence of Theorem 5.8 we obtain the following interpolation result. Let $S \in \operatorname{Mar}_{\infty, \Gamma}^{*}$ be a closed subset of a space $X \in \operatorname{Mar}_{\infty}^{*}$ and the weak $\infty$-Markov structure on $S$ is induced by that on $X$, i.e., the embedding $S \hookrightarrow X$ induces surjective morphism $\Omega_{X}^{0} \rightarrow \Omega_{S}^{0}$ of sheaves of germs of $C^{\infty}$ functions.

Corollary 5.11. There exists an open neighbourhood $N \subset X$ of $S$ such that for each $d$-closed form $\omega \in \Omega^{p}(S)$ there exists a d-closed form $\widetilde{\omega} \in \Omega^{p}(N)$ such that $\left.\widetilde{\omega}\right|_{S}=\omega$.

By $H^{*}(S, \mathbb{R})$ we denote the ring of singular cohomology of $S \in \operatorname{Mar}_{\infty}^{*}$ endowed with $\smile$ product (see, e.g., Appendix A in [29] for definitions). Also we consider the ring $H_{d R}^{*}(S, \mathbb{R})$ endowed with $\wedge$ product. If $S$ is a $C^{\infty}$ manifold, then the classical de Rham theorem states that these two rings are isomorphic. This result can be extended:

Theorem 5.12. For $S \in \operatorname{Mar}_{\infty, \Gamma}^{*}$, there exists an isomorphism of rings $\Phi_{S}$ : $\left(H_{d R}^{*}(S, \mathbb{R}), \wedge\right) \rightarrow\left(H^{*}(S, \mathbb{R}), \smile\right)$ such that if $f: S_{1} \rightarrow S_{2}$ is a $C^{\infty}$ map of spaces in $\operatorname{Mar}_{\infty, \Gamma}^{*}$, then the following diagram is commutative:

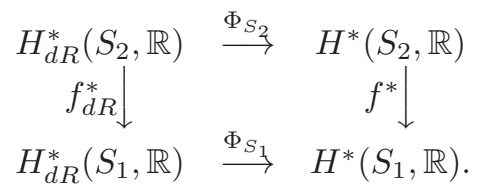

Here $f^{*}$ is the ring homomorphism induced by $f$.

Moreover, if $S$ is a $C^{\infty}$ manifold, then $\Phi_{S}$ coincides with the classical de Rham isomorphism defined by the pairing of differential forms and chains via integration. 
Let $S_{i} \in \operatorname{Mar}_{\infty, \Gamma}, i=1,2$. Then $S_{1} \times S_{2} \in \operatorname{Mar}_{\infty, \Gamma}$ as well. By $\pi_{i}: S_{1} \times S_{2} \rightarrow S_{i}$, $i=1,2$, we denote the natural projections. The map

$$
\left(\omega_{1}, \omega_{2}\right) \longmapsto \pi_{1}^{*}\left(\omega_{1}\right) \wedge \pi_{2}^{*}\left(\omega_{2}\right), \quad \omega_{i} \in \Omega^{*}\left(S_{i}\right), \quad i=1,2,
$$

determines a bilinear map $\wedge: H_{d R}^{*}\left(S_{1}, \mathbb{R}\right) \otimes H_{d R}^{*}\left(S_{2}, \mathbb{R}\right) \rightarrow H_{d R}^{*}\left(S_{1} \times S_{2}, \mathbb{R}\right)$ sending each $\oplus_{p+q=k} H_{d R}^{p}\left(S_{1}, \mathbb{R}\right) \otimes H_{d R}^{q}\left(S_{2}, \mathbb{R}\right)$ into $H_{d R}^{k}\left(S_{1} \times S_{2}, \mathbb{R}\right)$.

As a corollary of Theorem 5.12 we have the following generalization of the classical Künneth formula.

Theorem 5.13. Suppose $S_{2}$ is homotopy equivalent to a $C W$ complex having only finitely many cells in each dimension. Then the map $\wedge: H_{d R}^{*}\left(S_{1}, \mathbb{R}\right) \otimes H_{d R}^{*}\left(S_{2}, \mathbb{R}\right) \rightarrow$ $H_{d R}^{*}\left(S_{1} \times S_{2}, \mathbb{R}\right)$ is an isomorphism.

In case both $S_{i}$ admit locally finite acyclic open covers, the result was first established by Vladimir Goldshtein by the method analogous to the one described in Section 5 of [8] (by means of the Mayer-Vietoris sequences which clearly exist in the category of spaces in $\operatorname{Mar}_{\infty}^{*}$, cf. Section 2 of [8] for similar arguments). The question whether a general space in $\operatorname{Mar}_{\infty, \Gamma}$ has such a cover is open.

Theorem 5.13 can be applied, e.g., if $S_{2} \in \operatorname{Mar}_{\infty, \Gamma}^{*}$ is a compact topological manifold (in this case it has homotopy type of a finite $C W$ complex, see [24]).

In a forthcoming paper we present some results, different from those formulated above, on the de Rham cohomology of certain topological manifolds in $\operatorname{Mar}_{\infty}^{*} \backslash \operatorname{Mar}_{\infty, \Gamma}^{*}$ (including von Koch snowflake-type curves) and of Cantor-type sets in $\mathbb{R}^{n}$ (having similar to the Sierpiński gasket structures).

\section{Proofs of Properties (6), (10) and (11)}

\subsection{Proof of Property (6)}

We use the Remez-type inequality of [4]:

Let $V \subset \mathbb{R}^{n}$ be a bounded convex body and $\omega \subset V$ be a measurable subset. By $|\cdot|$ we denote the Lebesgue measure on $\mathbb{R}^{n}$. For each $p \in \mathcal{P}_{k, n}$,

$$
\sup _{V}|p| \leq T_{k}\left(\frac{1+\beta_{n}(\lambda)}{1-\beta_{n}(\lambda)}\right) \sup _{\omega}|p|,
$$

where $\lambda:=|\omega| /|V|, \beta_{n}(\lambda):=(1-\lambda)^{1 / n}$ and $T_{k}(x):=\frac{1}{2}\left(\left(x+\sqrt{x^{2}-1}\right)^{k}+(x-\right.$ $\left.\left.\sqrt{x^{2}-1}\right)^{k}\right)$ is the Chebyshev polynomial of degree $k$.

(Using inequality (6.1) one easily shows that cubes $Q_{r}(x)$ in Definition 1.1 of a weak Markov point can be replaced by closed balls $\bar{B}_{r}(x)$ with respect to a norm on $\mathbb{R}^{n}$.)

We also use the Markov inequality asserting that

$$
\sup _{[a, b]}\left|p^{\prime}\right| \leq \frac{k^{2}}{b-a} \cdot \sup _{[a, b]}|p| \quad \text { for each } p \in \mathcal{P}_{k, 1} .
$$


Proof. First, we prove the result for $m=n$.

Let $x \in S$ be a weak $k$-Markov point such that $D \varphi(x)$ is invertible. Since the required statement is clearly true for invertible affine maps, making suitable affine transformations of $\mathbb{R}^{n}$, we may assume that $x=\varphi(x)$ and $D \varphi(x)$ is the identity map. Then in an open neighbourhood $U$ of $x$ we have

$$
\varphi(y)=y+s(y) \cdot\|y-x\|_{\infty}, \quad \text { where } \lim _{y \rightarrow x} s(y)=0 .
$$

Here $\|\cdot\|_{\infty}$ is the $\ell_{\infty}$ norm on $\mathbb{R}^{n}$.

Suppose that $\left\{r_{i}\right\}_{i \in \mathbb{N}}$ is a convergent to zero sequence of positive numbers such that all $Q_{r_{i}}(x) \subset U$ and

$$
\sup _{i \in \mathbb{N}}\left\{\sup _{p \in \mathcal{P}_{k, n} \backslash\{0\}}\left(\frac{\sup _{Q_{r_{i}}(x)}|p|}{\sup _{S \cap Q_{r_{i}}(x)}|p|}\right)\right\}=: c<\infty .
$$

From (6.3) we get

$$
\|\varphi(y)-y\|_{\infty} \leq s_{i} \cdot r_{i} \quad \text { for all } y \in Q_{r_{i}}(x)
$$

where $s_{i}:=\sup _{y \in Q_{r_{i}}(x)}\|s(y)\| \rightarrow 0$ as $i \rightarrow \infty$. This implies that

$$
\varphi\left(S \cap Q_{r_{i}}(x)\right) \subset Q_{r_{i}\left(1+s_{i}\right)}(x) .
$$

Now, from (6.3)-(6.6) using inequality (6.1), the intermediate value theorem and inequality (6.2) we deduce that, for each $p \in \mathcal{P}_{k, n}$,

$$
\sup _{Q_{r_{i}\left(1+s_{i}\right)}(x)}|p| \leq T_{k}\left(\frac{1+\left(\left(1+s_{i}\right)^{n}-1\right)^{1 / n}}{1-\left(\left(1+s_{i}\right)^{n}-1\right)^{1 / n}}\right) \sup _{Q_{r_{i}}(x)}|p|
$$$$
\leq c \cdot T_{k}\left(\frac{1+\left(\left(1+s_{i}\right)^{n}-1\right)^{1 / n}}{1-\left(\left(1+s_{i}\right)^{n}-1\right)^{1 / n}}\right) \sup _{S \cap Q_{r_{i}}(x)}|p|
$$$$
\leq c \cdot T_{k}\left(\frac{1+\left(\left(1+s_{i}\right)^{n}-1\right)^{1 / n}}{1-\left(\left(1+s_{i}\right)^{n}-1\right)^{1 / n}}\right)\left(\sup _{\varphi\left(S \cap Q_{r_{i}}(x)\right)}|p|+\frac{k^{2} \sqrt{n} \cdot s_{i} \cdot r_{i}}{r_{i}\left(1+s_{i}\right)} \sup _{Q_{r_{i}\left(1+s_{i}\right)}(x)}|p|\right) \text {. }
$$

Due to (6.6) inequality (6.7) implies that, for all sufficiently large $i$,

$$
\sup _{Q_{r_{i}\left(1+s_{i}\right)}(x)}|p| \leq \frac{c \cdot T_{k}\left(\frac{1+\left(\left(1+s_{i}\right)^{n}-1\right)^{1 / n}}{1-\left(\left(1+s_{i}\right)^{n}-1\right)^{1 / n}}\right)}{1-c \cdot T_{k}\left(\frac{1+\left(\left(1+s_{i}\right)^{n}-1\right)^{1 / n}}{1-\left(\left(1+s_{i}\right)^{n}-1\right)^{1 / n}}\right) \cdot \frac{k^{2} \sqrt{n} \cdot s_{i}}{1+s_{i}}} \cdot \sup _{\varphi(S) \cap Q_{r_{i}\left(1+s_{i}\right)}(x)}|p| .
$$

The first factor in the right-hand side of the last inequality tends to $c$ as $i$ tends to $\infty$. This shows that $x:=\varphi(x)$ is a weak $k$-Markov point of $\varphi(S)$.

The case of $m<n$ is reduced to the previous one. Indeed, the statement is clearly true for linear projections $\mathbb{R}^{n} \rightarrow \mathbb{R}^{m}$. Making suitable affine transformations of $\mathbb{R}^{n}$ and $\mathbb{R}^{m}$ without loss of generality we may assume that $x=\varphi(x)$ 
and $D \varphi(x): \mathbb{R}^{n} \rightarrow \mathbb{R}^{m}$ is the linear projection sending $\left(y_{1}, \ldots, y_{n}\right) \in \mathbb{R}^{n}$ to $\left(y_{1}, \ldots, y_{m}\right) \in \mathbb{R}^{m}$. Let us define $\widetilde{\varphi}: \mathbb{R}^{n} \rightarrow \mathbb{R}^{n}$ by the formula

$$
\widetilde{\varphi}\left(y_{1}, \ldots, y_{n}\right):=\left(\varphi\left(y_{1}, \ldots, y_{n}\right), y_{m+1}, \ldots, y_{n}\right) \text {. }
$$

By the definition $D \widetilde{\varphi}(x)$ exists and is the identity map. Thus the previous case implies that $\widetilde{\varphi}(x)$ is a weak $k$-Markov point of $\widetilde{\varphi}(S) \subset \mathbb{R}^{n}$. Applying now the linear projection $D \varphi(x)$ to $\widetilde{\varphi}(x)$ and $\widetilde{\varphi}(S)$ we obtain the required result.

The second part of the statement of the property follows directly from the first one and the definition of weak $k$-Markov sets.

\subsection{Proof of Property (10)}

For each $k \in \mathbb{Z}_{+}$we construct a compact set $S \in \operatorname{Mar}_{k}^{*}(\mathbb{R}) \backslash \operatorname{Mar}_{k+1}^{*}(\mathbb{R})$.

For $k=0$ we define $S:=\{0\} \subset \mathbb{R}$. Clearly, $S \in \operatorname{Mar}_{0}^{*}(\mathbb{R}) \backslash \operatorname{Mar}_{1}^{*}(\mathbb{R})$, see properties (1), (2) of Section 2.

Assume now that $k \geq 1$. We set $S_{1}:=\{i / k, 0 \leq i \leq k\}$. Next, choose $\lambda_{1} \in(0,1 /(3 k)]$ and define $S_{2}:=S_{1}+\lambda_{1} \cdot S_{1}$. Then choose $\lambda_{2} \in\left(0, \lambda_{1} /(3 k)\right]$ and define $S_{3}:=S_{2}+\lambda_{2} \cdot S_{1}$. Proceeding similarly at the $n^{\text {th }}$ step we define $S_{n}:=S_{n-1}+\lambda_{n-1} \cdot S_{1}$ for some $\lambda_{n-1} \in\left(0, \lambda_{n-2} /(3 k)\right]$, etc. (Here $X+Y:=$ $\{x+y ; x \in X, y \in Y\}$ for $X, Y \subset \mathbb{R}$.)

The required set $S$ is defined as the closure of $\cup_{n=1}^{\infty} S_{n}$.

By the definition, each element $x \in S$ is written as a (convergent) series

$$
x=\sum_{m=0}^{\infty} \frac{j_{m} \cdot \lambda_{m}}{k},
$$

where $j_{m}$ are integers between 0 and $k$, and $\lambda_{0}:=1$. Moreover, $x \in S_{n}$ if and only if $j_{m}=0$ for all $m \geq n$.

Let us show that presentation (6.8) is unique. Indeed, suppose that $x=$ $\sum_{m=0}^{\infty} j_{m}^{\prime} \cdot \lambda_{m} / k$ is another such presentation. By $M \in \mathbb{Z}_{+}$we denote the minimal number such that $j_{m}=j_{m}^{\prime}$ for $0 \leq m \leq M$ but $j_{M+1} \neq j_{M+1}^{\prime}$. Then we have

$$
\begin{aligned}
0 & =\left|\sum_{m=0}^{\infty} \frac{\left(j_{m}-j_{m}^{\prime}\right) \cdot \lambda_{m}}{k}\right| \geq \frac{\left|j_{M+1}-j_{M+1}^{\prime}\right| \cdot \lambda_{M+1}}{k}-\sum_{m=M+2}^{\infty} \frac{\left|j_{m}-j_{m}^{\prime}\right| \cdot \lambda_{m}}{k} \\
& \geq \frac{\lambda_{M+1}}{k}-\sum_{m=M+2}^{\infty} \lambda_{m} \geq \frac{\lambda_{M+1}}{k}-\sum_{m=1}^{\infty} \frac{\lambda_{M+1}}{(3 k)^{m}} \geq \frac{\lambda_{M+1}}{2 k}>0,
\end{aligned}
$$

a contradiction proving the claim.

Proposition 6.1. Suppose that $\lim _{i \rightarrow \infty} \frac{\lambda_{i+1}}{\lambda_{i}}=0$. Then $S \in \operatorname{Mar}_{k}^{*}(\mathbb{R}) \backslash \operatorname{Mar}_{k+1}^{*}(\mathbb{R})$.

Proof. First, we reformulate property (9) of Section 2 for the case of weak Markov subsets of $\mathbb{R}$. 
Lemma 6.2. A point $x$ of a subset $S \subset \mathbb{R}$ is weak $k$-Markov if and only if there exist a convergent to 0 sequence of positive numbers $\left\{r_{i}\right\}_{i \in \mathbb{N}}$, a sequence of finite subsets $F_{i}:=\left\{x_{i 1}, \ldots, x_{i k+1}\right\} \subset S \cap Q_{r_{i}}(x), i \in \mathbb{N}$, and a positive number $\delta(x)$ such that for all $i \in \mathbb{N}$

$$
\min _{m \neq n}\left|x_{i m}-x_{i n}\right| \geq \delta(x) r_{i} .
$$

Proof of Lemma 6.2. According to property (9) it suffices to show that the hypothesis of the lemma is equivalent to (2.1). So assuming that (2.1) is valid, let us show that $(6.10)$ is also valid for some $\delta(x)>0$.

If, on the contrary, such $\delta(x)$ does not exist, then passing if necessary to a subsequence of $\left\{r_{i}\right\}_{i \in \mathbb{N}}$ without loss of generality we may assume that

(a) $\lim _{i \rightarrow \infty} \frac{x_{i m}-x_{i n}}{r_{i}}=0$ for some fixed $m \neq n$;

(b) $\lim _{i \rightarrow \infty} \frac{x_{i j}-x}{r_{i}}=: x_{j} \in[-1,1]$ for all $1 \leq j \leq k+1$.

Due to (a) the set $F:=\left\{x_{1}, \ldots, x_{k+1}\right\}$ consists of at most $k$ distinct points. Thus there exists a polynomial $p$ of degree $k$ on $\mathbb{R}$ having zeros at points of $F$ and such that $\max _{[-1,1]}|p|=1$. This and (b) imply for $p_{i}(t):=p\left(\frac{t-x}{r_{i}}\right) \in \mathcal{P}_{k, 1} \backslash\{0\}, i \in \mathbb{N}$, that

$$
\sup _{Q_{r_{i}}(x)}\left|p_{i}\right|=1 \quad \text { and } \quad \lim _{i \rightarrow \infty} \sup _{F_{i}}|p|=0,
$$

a contradiction with $(2.1)$.

Conversely, if (6.10) is valid then according to an inequality following from the classical Cartan lemma, see page 21 in [25], condition (2.1) is also valid for the same $\left\{r_{i}\right\}_{i \in \mathbb{N}}$ and $F_{i} \subset Q_{r_{i}}(x), i \in \mathbb{N}$.

Returning to the proof of Proposition 6.1, first, let us show that $S \notin \operatorname{Mar}_{k+1}^{*}(\mathbb{R})$. Assuming the contrary, we find, by Lemma 6.2 , a point $x \in S$, a convergent to 0 sequence of positive numbers $\left\{r_{i}\right\}_{i \in \mathbb{N}}$, a sequence of subsets $F_{i}:=\left\{x_{i 1}, \ldots, x_{i k+2}\right\} \subset$ $S \cap Q_{r_{i}}(x), i \in \mathbb{N}$, and a positive number $\delta(x)$ such that for all $i \in \mathbb{N}$

$$
\min _{m \neq n}\left|x_{i m}-x_{i n}\right| \geq \delta(x) r_{i}
$$

By $j(i), i \in \mathbb{N}$, we denote a natural number such that

$$
\lambda_{j(i)+1} \leq r_{i}<\lambda_{j(i)} .
$$

Case I. $\quad \lim _{i \rightarrow \infty} \frac{r_{i}}{\lambda_{j(i)+1}}<\infty \quad$ or $\quad \varliminf_{i \rightarrow \infty} \frac{\lambda_{j(i)}}{r_{i}}<\infty$.

In each of these cases passing to a subsequence of $\left\{r_{i}\right\}_{i \in \mathbb{N}}$ we may assume without loss of generality that there exist constants $c_{1}, c_{2}>0$ and a sequence $\{k(i)\}_{i \in \mathbb{N}} \subset \mathbb{N}$ such that for all $i \in \mathbb{N}$

$$
c_{1} \cdot \lambda_{k(i)} \leq r_{i} \leq c_{2} \cdot \lambda_{k(i)} .
$$

Next, according to (6.8), there exists a unique nondecreasing sequence $\left\{x_{i}\right\}_{i \in \mathbb{N}}$ such that $x_{i} \in S_{i}$ and $\left|x_{i+1}-x_{i}\right| \leq \lambda_{i}$ for all $i \in \mathbb{N}$, and $\lim _{i \rightarrow \infty} x_{i}=x$. 
Lemma 6.3. For all sufficiently large $i, S \cap Q_{r_{i}}(x)$ belongs to the set

$$
S\left(x_{k(i)}\right):=\left\{z \in S ; z:=x_{k(i)}+\sum_{m=k(i)}^{\infty} \frac{j_{m} \cdot \lambda_{m}}{k}, \quad j_{m} \in \mathbb{Z}_{+} \cap[0, k], m \geq k(i)\right\} .
$$

Proof of Lemma 6.3. If $z \notin S\left(x_{k(i)}\right)$, then one of the first $k(i)$ terms in (6.8) for $z$ differs from the corresponding term in (6.8) for $x_{k(i)}$. Thus, as in (6.9) we have

$$
|z-x| \geq\left|z-x_{k(i)}\right|-\left|x_{k(i)}-x\right| \geq \frac{\lambda_{k(i)-1}}{2 k}-\sum_{m=k(i)}^{\infty} \lambda_{m} \geq \frac{\lambda_{k(i)-1}}{2 k}-\frac{3 \lambda_{k(i)}}{k} .
$$

The latter, due to the hypothesis of the proposition and (6.13), is greater than $r_{i}$ for all sufficiently large $i$. This implies that $z \notin S \cap Q_{r_{i}}(x)$.

Passing to a subsequence of $\left\{r_{i}\right\}_{i \in \mathbb{N}}$, if necessary, we may assume without loss of generality that $S \cap Q_{r_{i}}(x) \subset S\left(x_{k(i)}\right)$ for all $i$.

According to the lemma, numbers in (6.11) can be written as

$$
x_{i p}:=x_{k(i)}+\sum_{m=k(i)}^{\infty} \frac{j_{m p} \cdot \lambda_{m}}{k}, \quad j_{m p} \in \mathbb{Z}_{+} \cap[0, k], m \geq k(i), 1 \leq p \leq k+2 .
$$

In particular, there exist integers $1 \leq p_{1}(i)<p_{2}(i) \leq k+2$ such that $j_{k(i) p_{1}(i)}=$ $j_{k(i) p_{2}(i)}$. This, the hypothesis of the proposition and (6.13) imply that for all sufficiently large $i$

$$
\left|x_{i p_{1}(i)}-x_{i p_{2}(i)}\right| \leq \sum_{m=k(i)+1}^{\infty} \lambda_{m} \leq \frac{3 \lambda_{k(i)+1}}{k}<\delta(x) \cdot r_{i}
$$

which contradicts $(6.11)$.

Case II. $\quad \lim _{i \rightarrow \infty} \frac{r_{i}}{\lambda_{j(i)+1}}=\infty \quad$ and $\quad \lim _{i \rightarrow \infty} \frac{\lambda_{j(i)}}{r_{i}}=\infty$.

Using the second of these conditions and arguing as in the proof of Lemma 6.3 we obtain that for all sufficiently large $i, S \cap Q_{r_{i}}(x) \subset S\left(x_{j(i)+1}\right)$ (see (6.12)). Then for the linear polynomial $p(t):=t-x, t \in \mathbb{R}$, we have

$$
\sup _{S \cap Q_{r_{i}}(x)}|p| \leq \sup _{S\left(x_{j(i)+1}\right)}|p| \leq \sum_{m:=j(i)+1}^{\infty} \lambda_{m} \leq \frac{3 \lambda_{j(i)+1}}{k} .
$$

On the other hand,

$$
\sup _{Q_{r_{i}}(x)}|p|=r_{i}
$$

Therefore

$$
\varliminf_{i \rightarrow \infty} \frac{\sup _{Q_{r_{i}}(x)}|p|}{\sup _{S \cap Q_{r_{i}}(x)}|p|} \geq \underset{i \rightarrow \infty}{\lim } \frac{k \cdot r_{i}}{3 \lambda_{j(i)+1}}=\infty,
$$

contradicting (6.11) (see the proof of Lemma 6.2).

Thus we have proved that $S$ is not weak $(k+1)$-Markov. 
To show that $S \in \operatorname{Mar}_{k}^{*}(\mathbb{R})$ it suffices to check the condition of Lemma 6.2 for points $x \in \cup_{i=1}^{\infty} S_{i}$ (a dense subset of $S$ ). In this case if $x \in S_{m}$ and $i \geq m$, then $S \cap Q_{\lambda_{i}}(x)$ contains the set $\{x\}+\lambda_{i} \cdot S_{1}$ of cardinality $k+1$ with distances between its distinct points being at least $\lambda_{i} / k$. Thus according to Lemma 6.2 , each $x \in \cup_{i=1}^{\infty} S_{i}$ is weak $k$-Markov. This gives the required statement and completes the proof of Proposition 6.1.

Remark 6.4. The constructed set $S$ is of Hausdorff dimension zero. For otherwise, due to property (8), see Section $2, S$ contains a nonempty weak $(k+1)$-Markov subset. However, each point of $S$ is at most weak $k$-Markov (see the proof of Proposition 6.1), a contradiction.

Taking direct products of $n$ copies of $S$ we obtain, according to property (5) of Section 2, a compact subset of $\mathbb{R}^{n}$ belonging to $\operatorname{Mar}_{k}^{*}\left(\mathbb{R}^{n}\right) \backslash \operatorname{Mar}_{k+1}^{*}\left(\mathbb{R}^{n}\right)$. This completes the proof of property (10).

\subsection{Proof of Property (11)}

First, we prove invariance of $\operatorname{Mar}_{k}^{*}(\mathbb{R})$ under bi-Lipschitz maps.

Theorem 6.5. If $x \in S \subset \mathbb{R}$ is a weak $k$-Markov point and $\varphi: S \rightarrow S^{\prime} \subset \mathbb{R}$ is a bi-Lipschitz map, than $\varphi(x) \in S^{\prime}$ is weak $k$-Markov. In particular, if $S$ is a weak $k$-Markov set, then $S^{\prime}$ is weak $k$-Markov as well.

Proof. Using the McShane extension theorem [27], without loss of generality we may assume that $\varphi: \mathbb{R} \rightarrow \mathbb{R}$ is Lipschitz and its restriction $\left.\varphi\right|_{S}: S \rightarrow S^{\prime}$ is biLipschitz. Let $\left\{r_{i}\right\}_{i \in \mathbb{N}}, F_{i}:=\left\{x_{i 1}, \ldots, x_{i k+1}\right\} \subset S \cap Q_{r_{i}}(x), i \in \mathbb{N}$, and $\delta(x)>0$ be the same as in Lemma 6.2 for the point $x \in S$. Then we have $\varphi\left(Q_{r_{i}}(x)\right) \subset$ $Q_{c_{1} r_{i}}(\varphi(x))$, where $c_{1}$ is the Lipschitz constant for $\varphi$, and for all $i \in \mathbb{N}$

$$
\min _{m \neq n}\left|\varphi\left(x_{i m}\right)-\varphi\left(x_{i n}\right)\right| \geq \frac{\delta(x)}{c_{2}} \cdot r_{i},
$$

where $c_{2}$ is the Lipschitz constant for $\varphi^{-1}: S^{\prime} \rightarrow S$. Thus $\varphi\left(F_{i}\right) \subset S^{\prime} \cap Q_{c_{1} r_{i}}(\varphi(x))$, $i \in \mathbb{N}$, satisfy conditions of Lemma 6.2 for the point $\varphi(x) \in S^{\prime}$, sequence $\left\{c_{1} r_{i}\right\}_{i \in \mathbb{N}}$ and $\delta(\varphi(x)):=\delta(x) /\left(c_{1} c_{2}\right)$. In particular, $\varphi(x) \in S^{\prime}$ is weak $k$-Markov.

The second statement follows from the first one because $\varphi$ is continuous and so sends dense subsets of $S$ to dense subsets of $S^{\prime}$.

To show that $\operatorname{Mar}_{k}^{*}\left(\mathbb{R}^{n}\right), n \geq 2, k \in \mathbb{N}$, is not invariant under bi-Lipschitz maps, we construct Lipschitz functions $\varphi: \mathbb{R} \rightarrow \mathbb{R}$ whose graphs $\Gamma_{\varphi} \in \operatorname{Mar}_{k}^{*}\left(\mathbb{R}^{2}\right) \backslash$ $\operatorname{Mar}_{k+1}^{*}\left(\mathbb{R}^{2}\right)$.

( $\star$ Let $f: \mathbb{R} \rightarrow \mathbb{R}$ be a bounded Lipschitz function such that

(a) a point $\left(x_{0}, f\left(x_{0}\right)\right) \in \Gamma_{f}$ is weak $k$-but not weak $(k+1)$-Markov, $1 \leq k<\infty$;

(b) $\left.f^{\prime}\right|_{\mathbb{R} \backslash\left\{x_{0}\right\}}$ has discontinuities of the first kind at some points of a set $\left\{x_{i}\right\}_{i \in \mathbb{N}}$;

(c) $f$ is differentiable at all points of the set $\mathbb{R} \backslash\left\{\left\{x_{i}\right\}_{i \in \mathbb{Z}_{+}}\right\}$.

Existence of such $f$ is established in Theorem 6.8. 
Suppose $\left\{c_{i}\right\}_{i \in \mathbb{N}}$ is a countable dense subset of an open interval $(-t, t) \subset \mathbb{R}$ such that

$$
\left\{x_{0}+c_{i}\right\}_{i \in \mathbb{N}} \bigcap\left\{x_{i}+c_{j}\right\}_{i, j \in \mathbb{N}}=\emptyset \text {. }
$$

(For instance, $\left\{x_{i}\right\}_{i \in \mathbb{Z}_{+}} \subset \mathbb{Q}$ and $\left\{c_{i}\right\}_{i \in \mathbb{N}}$ is a linearly independent family over $\mathbb{Q}$.)

Let $\left\{a_{i}\right\}_{i \in \mathbb{N}}$ be a sequence of nonzero numbers such that

$$
\sum_{i=1}^{\infty}\left|a_{i}\right|<\infty
$$

We define a bounded Lipschitz function

$$
\varphi(x):=\sum_{i=1}^{\infty} a_{i} f\left(x-c_{i}\right), \quad x \in\left[x_{0}-t, x_{0}+t\right] .
$$

Theorem 6.6. $\Gamma_{\varphi} \in \operatorname{Mar}_{k}^{*}\left(\mathbb{R}^{2}\right) \backslash \operatorname{Mar}_{k+1}^{*}\left(\mathbb{R}^{2}\right)$.

Proof. We start with:

Lemma 6.7. (1) If $f^{\prime}\left(x_{0}\right)$ exists, then the point $\left(x_{0}, f\left(x_{0}\right)\right) \in \Gamma_{f}$ is weak 0- but not weak 1-Markov.

(2) If $f^{\prime}\left(x_{0}+\right)$ and $f^{\prime}\left(x_{0}-\right)$ exist and distinct, then the point $\left(x_{0}, f\left(x_{0}\right)\right) \in \Gamma_{f}$ is weak 1- but not weak 2-Markov.

Proof of Lemma 6.7. (1) Assume that $f^{\prime}\left(x_{0}\right)$ exists. Consider a map $F: \mathbb{R}^{2} \rightarrow \mathbb{R}^{2}$,

$$
F(x, y):=(x, y-f(x)) .
$$

By our assumption the derivative $D F\left(x_{0}, f\left(x_{0}\right)\right)$ exists and has rank 2 . Thus according to property (6) of Section 2, $\left(x_{0}, f\left(x_{0}\right)\right) \in \Gamma_{f}$ is weak $k$-Markov if and only if $\left(x_{0}, 0\right):=F\left(x_{0}, f\left(x_{0}\right)\right) \in F\left(\Gamma_{f}\right):=\mathbb{R} \times\{0\}$ is weak $k$-Markov (with respect to $\left.\mathcal{P}_{k, 2}\right)$. Since $F\left(\Gamma_{f}\right)$ belongs to the zero set of the linear polynomial $y$, the point $F\left(x_{0}, f\left(x_{0}\right)\right)$ is 0 -Markov only. This gives the required statement for $\left(x_{0}, f\left(x_{0}\right)\right) \in \Gamma_{f}$.

(2) Suppose $f^{\prime}\left(x_{0}-\right) \neq f^{\prime}\left(x_{0}+\right)$ exist. Consider the function

$$
g(x):= \begin{cases}f\left(x_{0}\right)+f^{\prime}\left(x_{0}-\right)\left(x-x_{0}\right) & \text { for } x \leq x_{0} \\ f\left(x_{0}\right)+f^{\prime}\left(x_{0}+\right)\left(x-x_{0}\right) & \text { for } x>x_{0} .\end{cases}
$$

By the definition $\tilde{f}:=f-g$ has derivative 0 at $x_{0}$. Consider a map $\widetilde{F}: \mathbb{R}^{2} \rightarrow \mathbb{R}^{2}$,

$$
\widetilde{F}(x, y):=(x, y-\tilde{f}(x)) .
$$

Then $\widetilde{F}$ has derivative of rank 2 at each point $\left(x_{0}, y\right)$. In particular, $\left(x_{0}, f\left(x_{0}\right)\right) \in$ $\Gamma_{f}$ is weak $k$-Markov iff the point $\left(x_{0}, 0\right):=\widetilde{F}\left(x_{0}, f\left(x_{0}\right)\right) \in \widetilde{F}\left(\Gamma_{f}\right):=\Gamma_{g}$ is weak $k$-Markov. The last point is obviously 1-Markov, because

(a) the convex hull of the intersection of a square $Q$ centered at $\left(x_{0}, 0\right)$ with $\Gamma_{g}$ is a triangle $T(Q)$ such that the ratio of its area to the area of $Q$ is a positive constant independent of the size of the square; 
(b) maximum of modulus of a linear polynomial on $Q \cap \Gamma_{g}$ is equal to maximum of modulus of this polynomial on $T(Q)$;

(c) from (a) and Remez-type inequality (6.1) follow that maximum of modulus of a linear polynomial on $Q$ is bounded from above by an absolute constant times maximum of modulus of this polynomial on $T(Q)$.

To show that $\left(x_{0}, 0\right) \in \Gamma_{g}$ is not weak 2-Markov note that the nonzero quadratic polynomial $p(x, y):=\left(y-f\left(x_{0}\right)-f^{\prime}\left(x_{0}-\right)\left(x-x_{0}\right)\right)\left(y-f\left(x_{0}\right)-f^{\prime}\left(x_{0}+\right)\left(x-x_{0}\right)\right)$ is equal to zero on $\Gamma_{g}$. This gives the required statement.

To prove the theorem, first, let us show that each point $\left(x_{0}+c_{i}, \varphi\left(x_{0}+c_{i}\right)\right) \in \Gamma_{\varphi}$ is weak $k$ - but not weak $(k+1)$-Markov. Indeed, according to condition (6.14) all functions $a_{j} f\left(x-c_{j}\right), j \neq i$, are differentiable at $x_{0}+c_{i}$. Therefore the bounded Lipschitz function

$$
\varphi_{i}(x):=\sum_{j \neq i} a_{j} f\left(x-c_{j}\right), \quad x \in\left[x_{0}-t, x_{0}+t\right],
$$

is differentiable at $x_{0}+c_{i}$ as well.

Next, consider an invertible map $F:\left(x_{0}-t, x_{0}+t\right) \times \mathbb{R} \rightarrow\left(x_{0}-t, x_{0}+t\right) \times \mathbb{R}$ defined by

$$
F(x, y):=\left(x, y-\varphi_{i}(x)\right)
$$

Clearly, the derivative $D F\left(x_{0}+c_{i}, \varphi\left(x_{0}+c_{i}\right)\right)$ exists and has rank 2 . Thus according to property (6) of Section 2, the point $\left(x_{0}+c_{i}, \varphi\left(x_{0}+c_{i}\right)\right) \in \Gamma_{\varphi}$ is weak $m$-Markov iff $F\left(x_{0}+c_{i}, \varphi\left(x_{0}+c_{i}\right)\right) \in F\left(\Gamma_{\varphi}\right)$ is weak $m$-Markov. By the definition $F\left(\Gamma_{\varphi}\right)$ is the graph of the function $a_{i} f\left(x-c_{i}\right), x \in\left(x_{0}-t, x_{0}+t\right)$, and $F\left(x_{0}+c_{i}, \varphi\left(x_{0}+c_{i}\right)\right)=$ $\left(x_{0}+c_{i}, a_{i} f\left(x_{0}\right)\right)$. Applying to $F\left(\Gamma_{\varphi}\right)$ the invertible linear transformation of $\mathbb{R}^{2}$ sending each $(x, y)$ to $\left(x-c_{i}, y / a_{i}\right)$ we obtain that $\left(x_{0}+c_{i}, \varphi\left(x_{0}+c_{i}\right)\right) \in \Gamma_{\varphi}$ is weak $m$-Markov iff $\left(x_{0}, f\left(x_{0}\right)\right) \in \Gamma_{f}$ is weak $m$-Markov. The above argument and the hypothesis of the theorem imply that each $\left(x_{0}+c_{i}, \varphi\left(x_{0}+c_{i}\right)\right) \in \Gamma_{\varphi}$ is weak $k$ - but not weak $(k+1)$-Markov.

Now, consider points $\left(x_{i}+c_{j}, \varphi\left(x_{i}+c_{j}\right)\right) \in \Gamma_{\varphi}, i, j \in \mathbb{N}$. Due to condition (6.14), for a fixed $n \in \mathbb{N}$ either $a_{n} f^{\prime}\left(x_{i}+c_{j}-c_{n}\right)$ or $a_{n} f^{\prime}\left(x_{i}+c_{j}-c_{n}+\right) \neq a_{n} f^{\prime}\left(x_{i}+c_{j}-c_{n}-\right)$ exist. Hence, the same holds for $\varphi$, i.e., either $\varphi^{\prime}\left(x_{i}+c_{j}\right)$ or $\varphi^{\prime}\left(x_{i}+c_{j}-\right) \neq$ $\varphi^{\prime}\left(x_{i}+c_{j}+\right)$ exist. Then Lemma 6.7 implies that points $\left(x_{i}+c_{j}, \varphi\left(x_{i}+c_{j}\right)\right) \in \Gamma_{\varphi}$, $i, j \in \mathbb{N}$, are at most weak 1 -Markov.

Finally, by the hypothesis, $\varphi$ is differentiable on the set $\left[x_{0}-t, x_{0}+t\right] \backslash\left\{\left\{x_{i}+\right.\right.$

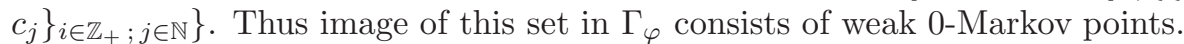

Combining these facts and using that $\left\{x_{0}+c_{i}\right\}_{i \in \mathbb{N}}$ is dense in $\left[x_{0}-t, x_{0}+t\right]$ and $k \geq 1$, we obtain that $\Gamma_{\varphi} \in \operatorname{Mar}_{k}^{*}\left(\mathbb{R}^{2}\right) \backslash \operatorname{Mar}_{k+1}^{*}\left(\mathbb{R}^{2}\right)$ as required.

Theorem 6.8. For each $k \in \mathbb{N}$ there exists a bounded Lipschitz function $f: \mathbb{R} \rightarrow \mathbb{R}$ satisfying assumptions (a)-(c) of condition ( $($ ).

Proof. For $k=1$ we define the function $f$ by the formula

$$
f(x):=\left\{\begin{aligned}
\left|x-x_{0}\right| & \text { for }\left|x-x_{0}\right| \leq 1 \\
1 & \text { for }\left|x-x_{0}\right|>1
\end{aligned}\right.
$$


Then $f^{\prime}$ has discontinuities of the first kind at points $x_{0} \pm 1$ and $x_{0}$ and according to Lemma 6.7, $\left(x_{0}, f\left(x_{0}\right)\right) \in \Gamma_{f}$ is weak 1- but not weak 2-Markov.

Now, we consider the case of $k \geq 2$. We set $t_{n}:=2^{-n^{2}}, n \in \mathbb{N}_{+}$. Let $p$ be a univariate polynomial of degree $k$ equals zero at $t_{0}:=1$ and $t_{1}:=1 / 2$ and such that $\sup _{[-1,1]}|p| \leq 1$. (If $k \geq 4$, then we can choose $p$ so that its derivatives at $t_{0}$ and $t_{1}$ are zeros as well.) We define the function $f$ by the formula

$$
f(x):=\left\{\begin{array}{cl}
0 & \text { if } x \in\left(\bigcup_{j \in \mathbb{Z}_{+}}\left[t_{2 j} / 2, t_{2 j+2}\right]\right) \cup(\mathbb{R} \backslash(0,1)), \\
t_{2 j} \cdot p\left(t_{2 j}^{-1} \cdot x\right) & \text { if } x \in\left[t_{2 j}, t_{2 j} / 2\right] ; j \in \mathbb{Z}_{+} .
\end{array}\right.
$$

According to this formula, $f$ is Lipschitz and bounded on $\mathbb{R}$ and $f^{\prime}$ has at most first kind discontinuities at points $t_{2 j}$ and $t_{2 j} / 2, j \in \mathbb{Z}_{+}$. (If $k \geq 4$ and $p^{\prime}$ is zero at $t_{0}$ and $t_{1}$, then $f^{\prime}$ is continuous on $\mathbb{R} \backslash\{0\}$.) Let us show that $(0, f(0))=(0,0) \in \Gamma_{f}$ is weak $k$-Markov.

Lemma 6.9. Let $\Gamma_{h} \subset Q_{1}(0)$ be the graph of $h:=\left.f\right|_{[1 / 3,1]}$. Then

$$
\sup _{q \in \mathcal{P}_{k, 2} \backslash\{0\}}\left(\frac{\sup _{Q_{1}(0)}|q|}{\sup _{\Gamma_{h}}|q|}\right)=: C<\infty .
$$

Proof of Lemma 6.9. Assuming, on the contrary, that the statement is false, using a compactness argument we find a polynomial $q \in \mathcal{P}_{k, 2} \backslash\{0\}$ such that $\left.q\right|_{\Gamma_{h} \cap Q_{1}(0)}=0$. Therefore considering $p$ and $q$ in complex variables $(z, w) \in \mathbb{C}^{2}$ we obtain that $q(z, w)$ equals zero on the complex irreducible curves $\left\{(z, w) \in \mathbb{C}^{2}\right.$; $w=p(z)\}$ and $\left\{(z, w) \in \mathbb{C}^{2} ; w=0\right\}$. Since $p$ is of degree $k$, for each $w$ from a Zariski open subset $U$ of $\mathbb{C}$ the equation $p(z)=w$ has $k$ distinct solutions $z_{1}(w), \ldots, z_{k}(w) \in \mathbb{C}$. Thus the univariate holomorphic polynomial $q(\cdot, w)$ being of degree at most $k$ has roots $z_{1}(w), \ldots, z_{k}(w)$. This implies that $q(z, w)=$ $r(w)(p(z)-w)$ for all $z \in \mathbb{C}$ and $w \in U$. Since $d^{k} q / d z^{k}=$ const, we get that $r$ is constant on the (connected) open set $U$. Also, $q(z, 0)=0$ for all $z \in \mathbb{C}$. Hence, $r=0$ (because $p \not \equiv 0$ ). We conclude that $q \equiv 0$, a contradiction.

Let us consider squares $Q_{t_{2 j}}(0) \subset \mathbb{R}^{2}, j \in \mathbb{Z}_{+}$. Observe that, by the definition of $f$, the graph of $t_{2 j}^{-1} \cdot f\left(t_{2 j} x\right), x \in[1 / 3,1]$, coincides with $\Gamma_{h}$ for all $j \in \mathbb{Z}_{+}$. Hence, by Lemma 6.9 for each $q \in \mathcal{P}_{k, 2} \backslash\{0\}$ we have

$$
\frac{\sup _{Q_{t_{2 j}}(0)}|q|}{\sup _{\Gamma_{f} \cap Q_{t_{2 j}}(0)}|q|} \leq \frac{\sup _{(x, y) \in Q_{1}(0)}\left|q\left(t_{2 j} x, t_{2 j} y\right)\right|}{\sup _{(x, y) \in \Gamma_{h} \cap Q_{1}(0)}\left|q\left(t_{2 j} x, t_{2 j} y\right)\right|} \leq C .
$$

This and Definition 1.1 imply that $(0,0) \in \Gamma_{f}$ is weak $k$-Markov.

Now, let us show that $(0,0) \in \Gamma_{f}$ is not weak $(k+1)$-Markov. Suppose that this is wrong. Then there exists a convergent to zero sequence of positive numbers $\left\{r_{i}\right\}_{i \in \mathbb{N}}$ such that

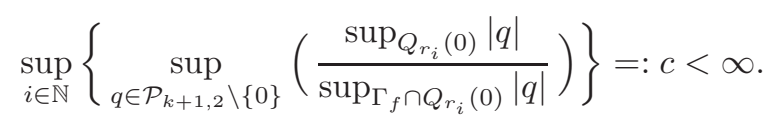

For each $i$ by $t_{2 j(i)} \in\left\{t_{2 j}\right\}_{j \in \mathbb{Z}_{+}}$we denote the maximal number not exceeding $r_{i}$. 
Applying (6.16) to $q(x, y):=y$ we obtain

$$
\sup _{i \in \mathbb{N}}\left\{\frac{\sup _{(x, y) \in Q_{r_{i}}(0)}|y|}{\sup _{(x, y) \in \Gamma_{f} \cap Q_{r_{i}}(0)}|y|}\right\}=\sup _{i \in \mathbb{N}}\left\{\frac{r_{i}}{t_{2 j(i)}}\right\} \leq c .
$$

Thus

$$
t_{2 j(i)} \leq r_{i} \leq c t_{2 j(i)} \quad \text { for all } i \in \mathbb{N} .
$$

Consider the polynomial $q_{i}(x, y):=y\left(y-t_{2 j(i)} \cdot p\left(t_{2 j(i)}^{-1} \cdot x\right)\right) \in \mathcal{P}_{k+1,2}$. Then $\sup _{Q_{r_{i}}(0)}\left|q_{i}\right| \geq\left|q_{i}\left(r_{i}, t_{2 j(i)}\right)\right|=r_{i}^{2}$. Also, inequality (6.17) and definition of the sequence $\left\{t_{n}\right\}$ imply that for all sufficiently large $i$,

$$
\frac{1}{2} \cdot t_{2(j(i)-1)} \geq c t_{2 j(i)} \geq r_{i}
$$

By the definition of $f$ for such $i$ we have

$$
\Gamma_{f} \cap\left\{\left[t_{2 j(i)}, r_{i}\right] \times\left[-r_{i}, r_{i}\right]\right\} \subset\left\{(x, y) \in \mathbb{R}^{2} ; y=0\right\} .
$$

In turn, this implies that (recall that $\sup _{[-1,1]}|p| \leq 1$ )

$$
\begin{aligned}
\sup _{\Gamma_{f} \cap Q_{r_{i}}(0)}\left|q_{i}\right| & =\sup _{\Gamma_{f} \cap\left\{\left[0, t_{2(j(i)+1)}\right] \times\left[-r_{i}, r_{i}\right]\right\}}\left|q_{i}\right| \\
& =\sup _{\Gamma_{f} \cap Q_{t_{2(j(i)+1)}}(0)}\left|q_{i}\right| \leq \sup _{Q_{t_{2(j(i)+1)}}(0)}\left|q_{i}\right| \leq 2 \cdot t_{2 j(i)} \cdot t_{2(j(i)+1)} .
\end{aligned}
$$

From these estimates we obtain

$$
\varliminf_{i \rightarrow \infty}\left(\frac{\sup _{Q_{r_{i}}(0)}\left|q_{i}\right|}{\sup _{\Gamma_{f} \cap Q_{r_{i}}(0)}\left|q_{i}\right|}\right) \geq \varliminf_{i \rightarrow \infty} \frac{r_{i}^{2}}{2 \cdot t_{2 j(i)} \cdot t_{2(j(i)+1)}} \geq \varliminf_{i \rightarrow \infty} \frac{t_{2 j(i)}}{2 \cdot t_{2(j(i)+1)}}=\infty .
$$

This contradicts $(6.16)$ and shows that $(0,0) \in \Gamma_{f}$ is not weak $(k+1)$-Markov.

Proposition 6.10. There exists a bounded Lipschitz function $\varphi: \mathbb{R} \rightarrow \mathbb{R}$ whose $\operatorname{graph} \Gamma_{\varphi} \in \operatorname{Mar}_{\infty}^{*}\left(\mathbb{R}^{2}\right)$.

Proof. According to Theorem 6.6, there exists a uniformly bounded sequence of Lipschitz functions $\left\{\varphi_{i}\right\}_{i \in \mathbb{N}}$ on $\mathbb{R}$ with uniformly bounded Lipschitz constants ${ }^{1}$ such that $\varphi_{i}$ is differentiable outside a countable dense subset $S_{i} \subset \mathbb{R}$ and $\Gamma_{\varphi_{i}} \subset \mathbb{R}^{2}$ is weak $i$-Markov, $i \in \mathbb{N}$. We choose a sequence of numbers $\left\{c_{i}\right\}_{i \in \mathbb{N}}$ such that $\left\{c_{i}+S_{i}\right\} \cap\left\{c_{j}+S_{j}\right\}=\emptyset$ for all $i \neq j$ (e.g., choose $\left\{c_{i}\right\}_{i \in \mathbb{N}}$ to be a linearly independent family over a vector space over $\mathbb{Q}$ generated by all $S_{i}, i \in \mathbb{N}$ ), and define a bounded Lipschitz function

$$
\varphi(x):=\sum_{i=1}^{\infty} \frac{\varphi_{i}\left(x-c_{i}\right)}{i^{2}} .
$$

Then $\varphi$ is differentiable outside the countable set $\cup_{i=1}^{\infty}\left\{S_{i}+c_{i}\right\}$. As in the proof of Theorem 6.6 one shows that $\Gamma_{\varphi}$ is weak $i$-Markov for all $i \in \mathbb{N}$ because each $\left\{S_{i}+c_{i}\right\}$ is dense in $\mathbb{R}$. Thus $\Gamma_{\varphi} \in \operatorname{Mar}_{\infty}^{*}\left(\mathbb{R}^{2}\right)$.

\footnotetext{
${ }^{1}$ For a Lipschitz function $f: \mathbb{R} \rightarrow \mathbb{R}$ its Lipschitz constant is $L(f):=\sup _{x \neq y}|f(x)-f(y)| /|x-y|$.
} 
Example 6.11. Point $(0,0) \in \mathbb{R}^{2}$ is weak $\infty$-Markov for the graph of $f(x)=$ $x \sin (1 / x)$ (exercise for the reader). However, it is weak 0 -Markov only for graphs of functions $g(x)=h(x) \sin (1 / x)$, where $h \in C\left(\mathbb{R}_{+}\right)$and $h(x)=o(x)$ as $x \rightarrow 0^{+}$.

Let us prove now that classes $\operatorname{Mar}_{k}^{*}\left(\mathbb{R}^{n}\right), n \geq 2, k \in \mathbb{N} \cup\{\infty\}$, are not invariant under bi-Lipschitz maps.

Let $\varphi: \mathbb{R} \rightarrow \mathbb{R}$ be a Lipschitz function such that $\Gamma_{\varphi} \in \operatorname{Mar}_{k}^{*}\left(\mathbb{R}^{2}\right)$ for some $k \in \mathbb{N} \cup\{\infty\}$. We set $S:=\Gamma_{\varphi} \times \mathbb{R}^{n-2} \subset \mathbb{R}^{n}$ (if $n=2$, then $S:=\Gamma_{\varphi}$ ). Due to property (5) of Section 2, $S \in \operatorname{Mar}_{k}^{*}\left(\mathbb{R}^{n}\right)$. We define a bi-Lipschitz map $F: \mathbb{R}^{n} \rightarrow \mathbb{R}^{n}$,

$$
F(x, y, z):=(x, y-\varphi(x), z), \quad(x, y) \in \mathbb{R}^{2}, z \in \mathbb{R}^{n-2} .
$$

Then $F(S):=\left\{(x, 0, z) \in \mathbb{R}^{n} ; x \in \mathbb{R}, z \in \mathbb{R}^{n-2}\right\}$. If $\operatorname{Mar}_{k}^{*}\left(\mathbb{R}^{n}\right)$ is invariant under $F$, then $F(S) \in \operatorname{Mar}_{k}^{*}\left(\mathbb{R}^{n}\right)$ as well. However, $F(S) \notin \operatorname{Mar}_{1}^{*}\left(\mathbb{R}^{n}\right)$ as the set of zeros of the linear polynomial $y \in \mathcal{P}_{1, n}$. This proves the second part of property (11).

\section{Proofs of Propositions 3.2, 3.3 and Theorem 3.4}

Proof of Proposition 3.2. First, let us show that the polynomial $T_{x}^{m}(f)$ in Definition 3.1 is unique. Suppose $\tilde{T}_{x}^{m}(f)$ and $T_{x}^{m}(f)$ are polynomials subject to this definition. Then $\left|\tilde{T}_{x}^{m}(f)(y)-T_{x}^{m}(f)(y)\right|=o\left(\|y-x\|_{\infty}^{m}\right)$. By the definition of a weak $k$-Markov point, there exists a convergent to zero sequence of positive numbers $\left\{r_{i}\right\}_{i \in \mathbb{N}}$ and a constant $c>0$ such that, for all $i$,

$$
\sup _{Q_{r_{i}}(x)}\left|\tilde{T}_{x}^{m}(f)-T_{x}^{m}(f)\right| \leq c \cdot \sup _{S \cap Q_{r_{i}}(x)}\left|\tilde{T}_{x}^{m}(f)-T_{x}^{m}(f)\right|
$$

On the other hand, by the one-dimensional Remez inequality (cf. (6.1)),

$$
\sup _{Q_{1}(x)}\left|\tilde{T}_{x}^{m}(f)-T_{x}^{m}(f)\right| \leq\left(\frac{4 \sqrt{n}}{r_{i}}\right)^{m} \cdot \sup _{Q_{r_{i}}(x)}\left|\tilde{T}_{x}^{m}(f)-T_{x}^{m}(f)\right| .
$$

Combining these inequalities we get

$$
\sup _{Q_{1}(x)}\left|\tilde{T}_{x}^{m}(f)-T_{x}^{m}(f)\right| \leq c \cdot\left(\frac{4 \sqrt{n}}{r_{i}}\right)^{m} \cdot o\left(r_{i}^{m}\right) \longrightarrow 0 \quad \text { as } \quad i \rightarrow \infty .
$$

Thus $\tilde{T}_{x}^{m}(f)=T_{x}^{m}(f)$.

Now, if $\tilde{f}: \mathbb{R}^{n} \rightarrow \mathbb{R}$ has derivatives of order $\leq m$ at $x$ and $T_{x}^{m}(\tilde{f})$ is its Taylor polynomial of degree $m$ at $x$, then due to Definition 3.1, the function $f:=\left.\tilde{f}\right|_{S}$ has derivatives of order $\leq m$ at $x$, and $T_{x}^{m}(f)=T_{x}^{m}(\tilde{f})$ by the uniqueness just proved. So let us prove the converse statement. To this end choose a retraction map $\psi: \mathbb{R}^{n} \rightarrow S$ such that $\|y-\psi(y)\|_{\infty} \leq 2 \cdot \operatorname{dist}_{\infty}(y, S)$ for all $y \in \mathbb{R}^{n}$. We define

$$
\begin{aligned}
& \hat{f}(y):=\frac{f(y)-T_{x}^{m}(f)(y)}{\|y-x\|_{\infty}^{m}}, \quad y \in S \backslash\{x\}, \quad \hat{f}(x):=0, \quad \text { and } \\
& \tilde{f}(y):=T_{x}^{m}(f)(y)+\|y-x\|_{\infty}^{m} \cdot(\hat{f} \circ \psi)(y), \quad y \in \mathbb{R}^{n} .
\end{aligned}
$$


Then $\tilde{f}$ is the required extension of $f$. To prove this we must check that $\hat{f} \circ \psi$ is continuous at $x$. (Since $(\hat{f} \circ \psi)(x)=0$, this will imply the required statement.)

For otherwise, there exists a sequence $\left\{y_{i}\right\}_{i \in \mathbb{N}} \subset \mathbb{R}^{n}$ tending to $x$ such that $\varlimsup_{i \rightarrow \infty}\left|(\hat{f} \circ \psi)\left(y_{i}\right)\right| \neq 0$. By definition, $\left\|y_{i}-\psi\left(y_{i}\right)\right\|_{\infty} \leq 2 \cdot \operatorname{dist}_{\infty}\left(y_{i}, S\right) \leq 2$. $\left\|y_{i}-x\right\|_{\infty}$. Hence, $\left\{\psi\left(y_{i}\right)\right\}_{i \in \mathbb{N}} \subset S$ tends to $x$ as well. But $\hat{f}$ is continuous (and equals zero) at $x$ by Definition 3.1. This implies that $\varlimsup_{i \rightarrow \infty}\left|(\hat{f} \circ \psi)\left(y_{i}\right)\right|=$ $\lim _{i \rightarrow \infty}\left|\hat{f}\left(\psi\left(y_{i}\right)\right)\right|=0$, a contradiction showing that $\hat{f} \circ \psi$ is continuous and equals zero at $x$.

Proof of Proposition 3.3. Let $\left\{r_{i}\right\}_{i \in \mathbb{N}}$ and $F_{i} \subset S \cap Q_{r_{i}}(x), \operatorname{card} F_{i}=\operatorname{dim} \mathcal{P}_{k, n}$, $i \in \mathbb{N}$, be the same as in property (9) for $x$, see (2.1). Perturbing each $F_{i}$, without loss of generality we may assume that $x \notin F_{i}$ for all $i \in \mathbb{N}$. Since inequalities of property (9) are also valid for polynomials in $\mathcal{P}_{m, n}$, by Proposition 3.5 in [3] there exist subsets $F_{i}^{m} \subset F_{i}$, card $F_{i}=\operatorname{dim} \mathcal{P}_{m, n}\left(=: d_{m, n}\right), i \in \mathbb{N}$, such that

$$
\sup _{i \in \mathbb{N}}\left\{\sup _{p \in \mathcal{P}_{m, n} \backslash\{0\}}\left(\frac{\sup _{Q_{r_{i}}(x)}|p|}{\sup _{F_{i}^{m}}|p|}\right)\right\}=: c<\infty .
$$

We set $\left\{r_{i}\right\}_{i \in \mathbb{N}}$ and $\left\{F_{i}^{m}\right\}_{i \in \mathbb{N}}$ to be the required objects of the proposition.

Next, from (7.1) follows that each $F_{i}^{m}$ is a minimal interpolating set for polynomials of degree $m$ on $\mathbb{R}^{n}$. If $F_{i}^{m}:=\left\{x_{i 1}, \ldots, x_{i d_{m, n}}\right\}$ by $\ell_{i j} \in \mathcal{P}_{m, n}$ we denote a polynomial equal to 1 at $x_{i j}$ and 0 at all other points $x_{i s}, s \neq j$. Then we define

$$
\mu_{i}^{\alpha}\left(\left\{x_{i j}\right\}\right):=\frac{1}{\alpha !} \cdot D^{\alpha} \ell_{i j}(x), \quad i \in \mathbb{N}, 1 \leq j \leq d_{m, n},|\alpha| \leq m .
$$

Let us prove (3.1). By definition,

$$
\begin{aligned}
\int_{F_{i}^{m}} f d \mu_{i}^{\alpha} & =\sum_{j=1}^{d_{m, n}} f\left(x_{i j}\right) \cdot \frac{D^{\alpha} \ell_{i j}(x)}{\alpha !}=\frac{1}{\alpha !} \cdot D^{\alpha}\left(\sum_{j=1}^{d_{m, n}} f\left(x_{i j}\right) \cdot \ell_{i j}\right)(x) \\
& =\frac{D^{\alpha}\left(P_{i}(f)\right)(x)}{\alpha !}
\end{aligned}
$$

where $P_{i}(f) \in \mathcal{P}_{m, n}$ is such that $P_{i}(f)\left(x_{i j}\right):=f\left(x_{i j}\right)$ for all $1 \leq j \leq d_{m, n}$. Thus to prove the required statement it suffices to show that the sequence of polynomials $\left\{P_{i}(f)\right\}_{i \in \mathbb{N}}$ converges (uniformly on a fixed cube) to $T_{x}^{m}$. To this end consider polynomials $P_{i}(f)-T_{x}^{m}, i \in \mathbb{N}$. Since $\left.P_{i}(f)\right|_{F_{i}^{m}}=f$, there exists a convergent to zero sequence of positive numbers $\left\{c_{i}\right\}_{i \in \mathbb{N}}$ such that, for all $i$,

$$
\max _{y \in F_{i}^{m}} \frac{\left|P_{i}(f)(y)-T_{x}^{m}(y)\right|}{\|y-x\|_{\infty}^{m}} \leq c_{i} .
$$

From these inequalities arguing as in the proof of Proposition 3.2 we obtain that

$$
\sup _{Q_{1}(x)}\left|P_{i}(f)-T_{x}^{m}\right| \leq \tilde{c}_{i}
$$

for some convergent to zero sequence $\left\{\tilde{c}_{i}\right\}_{i \in \mathbb{N}} \subset \mathbb{R}_{+}$. This shows that $\left\{P_{i}(f)\right\}_{i \in \mathbb{N}}$ converges uniformly on $Q_{1}(x)$ to $T_{x}^{m}$ and completes the proof. 
Proof of Theorem 3.4. Inequalities (3.2) and the Whitney-Glaeser theorem (see, e.g., Volume I, Section 2.2 in [2]) imply that there exists a function $F \in C^{m, \omega}\left(\mathbb{R}^{n}\right)$ of norm at most $c(m, n) \lambda$ such that $T_{x}^{m}(F)=T_{x}^{m}(f)$ for all weak $k$-Markov points $x \in S$. In particular, $F=f$ at weak $k$-Markov points of $S$. Since the set of such points is dense in $S$ and $f$ is continuous, $\left.F\right|_{S}=f$, i.e., $f \in C^{m, \omega}(S)$. Conversely, if $f \in C^{m, \omega}(S)$, then by the Whitney-Glaeser theorem it satisfies inequalities (3.2).

Also, due to Proposition 3.2, $T_{x}^{m}(f)$ coincides with $T_{x}^{m}(F)$ for any $C^{m, \omega}$ extension $F$ of $f$. Therefore by the Whitney-Glaeser theorem applied to an almost optimal $C^{m, \omega}$ extension of $f$, inf $\lambda \approx\|f\|_{C^{m, \omega}(S)}$ with constants of equivalence depending on $m$ and $n$ only.

\section{Proofs of Theorems 4.3, 4.4 and 4.5}

Proof of Theorem 4.3. Since $f \in C^{m+1}(S)$ and $S$ is compact, there exists a function $\hat{f} \in C^{m+1}\left(\mathbb{R}^{n}\right)$ with compact support such that $\left.\hat{f}\right|_{S}=f$. We prove the theorem by induction on $m$.

Assume first that $m=1$, i.e., $D_{S}^{\alpha} f=0$ for all $|\alpha|=1$. For each $D_{j} \subset \Omega$ consider the $d$-closed 1 -form $\lambda_{j}:=d\left(\left.\hat{f}\right|_{D_{j}}\right)$. We extend $\lambda_{j}$ to $\left(D_{j}\right)^{c}$ by zero denoting the extended form $\hat{\lambda}_{j}$.

Lemma 8.1. There exist a modulus of continuity $\omega$ and a constant $C>0$ such that if $\hat{\lambda}_{j}=\sum_{i=1}^{n} a_{i j} d x_{i}$, then all $a_{i j} \in C^{1, \omega}\left(\mathbb{R}^{n}\right)$ and $\sup _{\ell, 1 \leq i \leq n}\left\|\sum_{j=1}^{\ell} a_{i j}\right\|_{C^{1, \omega}\left(\mathbb{R}^{n}\right)} \leq$ $C$, and all $\hat{\lambda}_{j}$ are $d$-closed.

Proof of Lemma 8.1. Since $\hat{f} \in C_{c}^{2}\left(\mathbb{R}^{n}\right)$, there exists a modulus of continuity $\omega$ such that $\hat{f} \in C^{2, \omega}\left(\mathbb{R}^{n}\right)$. Let us prove the statement for such $\omega$ and $C:=\|\hat{f}\|_{C^{2, \omega}\left(\mathbb{R}^{n}\right)}$.

Let $d \hat{f}:=\sum_{i=1}^{n} c_{i} d x_{i}$. By the hypothesis, all $c_{i}$ and their partial derivatives $\partial c_{i} / \partial x_{j}$ vanish on $S$. Moreover, $\left.a_{i j}\right|_{D_{j}}:=\left.c_{i}\right|_{D_{j}}$. Hence, for all admissible $\ell \in \mathbb{N}$, $\sum_{j=1}^{\ell} a_{i j}(x)$ and $\sum_{j=1}^{\ell} \nabla a_{i j}(x)$ tend to 0 uniformly in $x$ as $D_{\ell}^{\prime}:=\cup_{j=1}^{\ell} D_{j} \ni x \rightarrow$ $\cup_{j=1}^{\ell}\left(\bar{D}_{j} \backslash D_{j}\right)(\subset S)$. This and the definition of $a_{i j}$ imply that $a_{i \ell}^{\prime}:=\sum_{j=1}^{\ell} a_{i j} \in$ $C_{c}^{1}\left(\mathbb{R}^{n}\right)$. Moreover, $\left\|a_{i \ell}^{\prime}\right\|_{C^{1, \omega}\left(D_{\ell}^{\prime}\right)}=\left\|\left.c_{i}\right|_{D_{\ell}^{\prime}}\right\|_{C^{1, \omega}\left(D_{\ell}^{\prime}\right)} \leq C$ and if $x_{1} \in D_{\ell}^{\prime}$ and $x_{2} \in\left(D_{\ell}^{\prime}\right)^{c}$, then

$$
\begin{aligned}
\sup _{\mathbb{R}^{n}}\left|a_{i \ell}^{\prime}\right| & +\max _{|\alpha|=1} \frac{\left|D^{\alpha} a_{i \ell}^{\prime}\left(x_{1}\right)-D^{\alpha} a_{i \ell}^{\prime}\left(x_{2}\right)\right|}{\omega\left(\left\|x_{2}-x_{1}\right\|_{\infty}\right)} \leq \sup _{\mathbb{R}^{n}}\left|c_{i}\right|+\max _{|\alpha|=1} \frac{\left|D^{\alpha} c_{i}\left(x_{1}\right)-D^{\alpha} c_{i}(z)\right|}{\omega\left(\left\|x_{2}-x_{1}\right\|_{\infty}\right)} \\
\leq & \left(1-\frac{\omega\left(\left\|z-x_{1}\right\|_{\infty}\right)}{\omega\left(\left\|x_{2}-x_{1}\right\|_{\infty}\right)}\right) \sup _{\mathbb{R}^{n}}\left|c_{i}\right| \\
& +\frac{\omega\left(\left\|z-x_{1}\right\|_{\infty}\right)}{\omega\left(\left\|x_{2}-x_{1}\right\|_{\infty}\right)}\left(\sup _{\mathbb{R}^{n}}\left|c_{i}\right|+\max _{|\alpha|=1} \frac{\left|D^{\alpha} c_{i}\left(x_{1}\right)-D^{\alpha} c_{i}(z)\right|}{\omega\left(\left\|z-x_{1}\right\|_{\infty}\right)}\right) \leq C
\end{aligned}
$$

for some $z \in\left[x_{1}, x_{2}\right] \cap \overline{D_{\ell}^{\prime}} \backslash D_{\ell}^{\prime}(\neq \emptyset)$.

These show that $a_{i j} \in C^{1, \omega}\left(\mathbb{R}^{n}\right)$ and $\sup _{\ell, 1 \leq i \leq n}\left\|a_{i \ell}^{\prime}\right\|_{C^{1, \omega}\left(\mathbb{R}^{n}\right)} \leq C$.

The closedness of $\hat{\lambda}_{j}$ follows from its definition. 
Let us fix a point $o \notin \bar{\Omega}$ and define

$$
g_{j}(x):=\int_{o}^{x} \hat{\lambda}_{j}, \quad x \in \mathbb{R}^{n}
$$

Since $\hat{\lambda}_{j}$ is $d$-closed, the integral is independent of the path joining $o$ and $x$. In particular, since by our assumption $\left(\bar{D}_{j}\right)^{c}$ is connected and $\partial D_{j}=\partial\left(\bar{D}_{j}\right)^{c}$, the continuous function $g_{j}$ is equal to zero on $\left(D_{j}\right)^{c}$ and $\sup _{\mathbb{R}^{n}}\left|g_{j}\right| \leq C^{\prime}$ for some $C^{\prime}>0$ independent of $j$. Next, due to Lemma 8.1 the function $g_{\ell}^{\prime}:=\sum_{j=1}^{\ell} g_{j}$ equals zero on $\left(D_{\ell}^{\prime}\right)^{c}$ and satisfies $\left\|g_{\ell}^{\prime}\right\|_{C^{2, \omega}\left(\mathbb{R}^{n}\right)} \leq C^{\prime \prime}$ for some $C^{\prime \prime}>0$ independent of $\ell$. Also, by our construction, $d\left(\left.\hat{f}\right|_{D_{\ell}^{\prime}}-g_{\ell}^{\prime}\right)=0$ for all $\ell$.

Further, according to a compactness result for bounded sequences in $C^{2, \omega}\left(\mathbb{R}^{n}\right)$, see, e.g. Volume II, Proposition 5.4 in [2], there exists a subsequence $\left\{g_{\ell_{p}}^{\prime}\right\}_{p \in P}$ of $\left\{g_{\ell}^{\prime}\right\}$ and a function $g \in C^{2, \omega}\left(\mathbb{R}^{n}\right)$ such that for every closed cube $Q \subset \mathbb{R}^{n}$,

$$
\lim _{p \in P}\left\|g-g_{\ell_{p}}^{\prime}\right\|_{C^{2}(Q)}=0 .
$$

In particular, $g$ equals zero on $\left(\cup_{j} D_{j}\right)^{c}$ and $d\left(\left.\hat{f}\right|_{\Omega}-\left.g\right|_{\Omega}\right)=0$ (since $\bar{\Omega}:=\left(\cup_{j} D_{j}\right) \sqcup S$ and $\left.d_{S}\left(\left.\hat{f}\right|_{S}\right)=0\right)$. Connectedness of $\Omega$ implies that $\left.\hat{f}\right|_{\bar{\Omega}}-\left.g\right|_{\bar{\Omega}}=$ const. On the other hand, $\left.\hat{f}\right|_{S}=f$ and $\left.g\right|_{S}=0$. This shows that $f$ is the restriction to $S$ of a (unique) polynomial of degree zero, as required. As a corollary, we get immediately that $S$ is connected. This proves the case $m=1$.

Now, assuming that the theorem is valid for $m-1 \geq 1$ we prove it for $m$.

To this aim we consider functions $D_{S}^{\alpha} f,|\alpha|=m-1$. Then $d_{S}\left(D_{S}^{\alpha} f\right)=0$ and by the induction hypothesis, $D_{S}^{\alpha} f=$ const for all $\alpha$. Since $D_{S}^{\alpha} f=D_{S}^{\pi(\alpha)} f$ for any permutation $\pi$ defined on the set of coordinates of $\alpha$, there exists a unique homogeneous polynomial $p \in \mathcal{P}_{m-1, n}$ such that $D^{\alpha} p=D_{S}^{\alpha} f$ for all $|\alpha|=m-1$. In particular, $D^{\alpha}\left(f-\left.p\right|_{S}\right)=0$ for all $|\alpha|=m-1$ and $f-\left.p\right|_{S} \in C^{m+1}(S)$. Applying to this function the induction hypothesis we obtain that $f-\left.p\right|_{S}=\left.q\right|_{S}$ for a unique $q \in \mathcal{P}_{m-2, n}$. Thus $f$ is the restriction to $S$ of the (unique) polynomial $p_{f}:=p+q \in \mathcal{P}_{m-1, n}$.

Proof of Theorem 4.4. Since $f \in C_{\mathbb{C}}^{2}(S)$ and $S$ is compact, there exist a modulus of continuity $\omega$ and a function $\hat{f} \in C_{\mathbb{C}}^{2, \omega}\left(\mathbb{C}^{n}\right):=C^{2, \omega}\left(\mathbb{R}^{2 n}\right) \otimes \mathbb{C}$ with compact support such that $\left.\hat{f}\right|_{S}=f$. We set $\eta:=\bar{\partial}\left(\left.\hat{f}\right|_{\bar{\Omega}}\right)$ and by $\hat{\eta}$ denote its extension to $\mathbb{C}^{n}$ by zero. Then repeating literally the proof of Lemma 8.1 we obtain that if $\hat{\eta}:=\sum_{j=1}^{n} a_{j} d \bar{z}_{j}$, then all $a_{j} \in C_{\mathbb{C}}^{1, \omega}\left(\mathbb{C}^{n}\right)$ and $\hat{\eta}$ is a $\bar{\partial}$-closed $(0,1)$-form on $\mathbb{C}^{n}$ with support in $\overline{\left(\cup_{j \geq 1} D_{j}\right)}$.

Lemma 8.2. There exists a function $g \in C_{\mathbb{C}}^{1}\left(\mathbb{C}^{n}\right)$ equals zero on $S \cup \bar{\Omega}^{c}$ such that $\bar{\partial} g=\hat{\eta}$.

Proof of Lemma 8.2. We set

$\delta(z):=d z_{1} \wedge \cdots \wedge d z_{n} \quad$ and $\quad \delta^{\prime}(z):=\sum_{k=1}^{n}(-1)^{k-1} d z_{1} \wedge \cdots \wedge d z_{k-1} \wedge d z_{k+1} \wedge \cdots \wedge d z_{n}$ 
Without loss of generality we may assume that $\bar{\Omega} \subset B$, the open unit Euclidean ball in $\mathbb{C}^{n}$. Then the function

$$
g(z):=\int_{\xi \in B} \hat{\eta}(\xi) \wedge \delta^{\prime}\left(\frac{\bar{\xi}-\bar{z}}{\|\xi-z\|^{2}}\right) \wedge \delta(\xi), \quad z \in \mathbb{C}^{n}
$$

satisfies $\bar{\partial}\left(\left.g\right|_{B}\right)=\left.\hat{\eta}\right|_{B}$, see Section 6.4, Theorem 6.9 in [17]; here $\|\cdot\|$ is the the $\ell_{2}$-norm on $\mathbb{C}^{n}$. Since supp $\hat{\eta} \Subset B, g \in C_{\mathbb{C}}^{1}\left(\mathbb{C}^{n}\right)$, and moreover, is holomorphic on $B \backslash \operatorname{supp} \hat{\eta}$. Further, $(8.1)$ shows that $g$ is complex analytic on $(\operatorname{supp} \hat{\eta})^{c}$ and $\lim _{z \rightarrow \infty} g(z)=0$. Therefore by the classical Hartogs theorem in $\mathbb{C}^{n}, n \geq 2$, the function $g$ is holomorphic on $(\operatorname{supp} \hat{\eta})^{c}$ and equals zero in the connected component of this set containing $(\bar{B})^{c}$.

On the other hand, we can write $\hat{\eta}=\sum_{j \geq 1} \hat{\eta}_{j}$, where $\hat{\eta}_{j}$ is extension by zero of $\left.\eta\right|_{D_{j}}$. Then we rewrite (8.1) as

$$
\begin{aligned}
g(z) & =\int_{\xi \in B} \sum_{j \geq 1} \hat{\eta}_{j}(\xi) \wedge \delta^{\prime}\left(\frac{\bar{\xi}-\bar{z}}{\|\xi-z\|^{2}}\right) \wedge \delta(\xi) \\
& =\sum_{j \geq 1} \int_{\xi \in B} \hat{\eta}_{j}(\xi) \wedge \delta^{\prime}\left(\frac{\bar{\xi}-\bar{z}}{\|\xi-z\|^{2}}\right) \wedge \delta(\xi) .
\end{aligned}
$$

(The change of the order is possible because the series on the right is absolutely convergent.) Since each $\hat{\eta}_{j}$ is of class $C^{1}$ and $\bar{\partial}$-closed on $\mathbb{C}^{n}$ (cf. the proof of Lemma 8.1) and $\left(\bar{D}_{j}\right)^{c}$ is connected and $\partial D_{j}=\partial\left(\bar{D}_{j}\right)^{c}$, each term, say $g_{j}$, on the right-hand side of the above identity is zero on $\left(D_{j}\right)^{c}$ and satisfies $\bar{\partial} g_{j}=\hat{\eta}_{j}$ on $\mathbb{C}^{n}$ (cf. a similar argument above for $g$ ). Hence, $g=\sum_{j>1} g_{j}$ equals zero on $\cup_{j \geq 1}\left(D_{j}\right)^{c}=S \sqcup \bar{\Omega}^{c}$ as required.

Now, consider the function $F:=\left.\hat{f}\right|_{\bar{\Omega}}-\left.g\right|_{\bar{\Omega}}$. Then by Lemma $8.2, F \in C_{\mathbb{C}}^{1}(\bar{\Omega})$, $\left.F\right|_{S}=f$ and $\left.\bar{\partial} F\right|_{\Omega}=0$. Thus $F \in \mathcal{O}(\Omega) \cap C_{\mathbb{C}}^{1}(\bar{\Omega})$. Further, by the maximum modulus principle, $\sup _{D_{j}}|F|=\sup _{\bar{D}_{j} \backslash D_{j}}|F|$ for all $j$. Moreover, $\cup_{j \geq 1}\left(\bar{D}_{j} \backslash D_{j}\right) \subset$ $S$ and $\bar{\Omega}:=\left(\cup_{j \geq 1} D_{j}\right) \cup S$. Therefore $\sup _{\bar{\Omega}}|F|=\sup _{S}|f|$. This implies that such an extension $F$ is unique.

Proof of Theorem 4.5. Let $\hat{f} \in C^{2, \alpha}\left(\mathbb{R}^{n}\right)$ be a function with compact support such that $\left.\hat{f}\right|_{S}=f$. Consider the functions $\tilde{f}_{1}$, obtained as extension of $\left.(\Delta \hat{f})\right|_{\left(\overline{\cup_{j} D_{j}}\right)^{c}}$ by zero to $\mathbb{R}^{n}$, and $\tilde{f}_{2}$, obtained as extension of $\left.(\Delta \hat{f})\right|_{\cup_{j} D_{j}}$ by zero to $\mathbb{R}^{n}$. Then from the hypotheses of the theorem as in the proof of Theorem 4.3 one obtains that $\tilde{f}_{i} \in C^{0, \alpha}\left(\mathbb{R}^{n}\right), i=1,2$, and have compact supports. By the definition, $\Delta \hat{f}=\tilde{f}_{1}+\tilde{f}_{2}$ (because $\left.\left.(\Delta \hat{f})\right|_{S}=0\right)$.

We set

$$
F_{i}(x):=\frac{1}{n(2-n) \omega_{n}} \int_{\mathbb{R}^{n}} \frac{\tilde{f}_{i}(y)}{\|x-y\|^{n-2}} d y_{1} \wedge \cdots \wedge d y_{n}
$$

where $\omega_{n}$ is the volume of the unit Euclidean ball in $\mathbb{R}^{n}$ and $\|\cdot\|$ is the $\ell_{2}$-norm on $\mathbb{R}^{n}$. Then according to the Schauder theorem, see, e.g., Chapter 4 in [14], 
$F_{i} \in C^{2, \alpha}\left(\mathbb{R}^{n}\right)$ and $\Delta F_{i}=\tilde{f}_{i}, i=1,2$. Moreover, $\lim _{x \rightarrow \infty} F_{i}=0$ (because $n \geq 3$ and $\tilde{f}_{i}$ have compact supports) and $F_{1}, F_{2}$ are harmonic in $\cup_{j \geq 1} D_{j}$ and $\left(\overline{\cup_{j \geq 1} D_{j}}\right)^{c}$, respectively. By our construction $\Delta\left(F_{1}+F_{2}-\hat{f}\right)=0$, i.e., $F_{1}+F_{2}-\hat{f}$ is a harmonic function having limit zero at infinity. Thus by the maximum principle $F_{1}+F_{2}-\hat{f}=0$. In particular, $\left.\left(F_{1}+F_{2}\right)\right|_{S}=f$, as required.

If $F_{1}^{\prime}, F_{2}^{\prime} \in C^{2, \alpha}\left(\mathbb{R}^{n}\right)$ is another pair of functions satisfying the conclusion of the theorem, then $F_{1}-F_{1}^{\prime}=F_{2}-F_{2}^{\prime}$ on $S$ and $F_{1}-F_{1}^{\prime}, F_{2}-F_{2}^{\prime}$ are harmonic in $\cup_{j \geq 1} D_{j}$ and $\left(\overline{\cup_{j \geq 1} D_{j}}\right)^{c}$, respectively. Since $F_{i}-F_{i}^{\prime} \in C^{2, \alpha}\left(\mathbb{R}^{n}\right)$ and $S$ is weak $k$ Markov with $k \geq 2$, the latter implies that the function $H \in C\left(\mathbb{R}^{n}\right)$ equals $F_{1}-F_{1}^{\prime}$ on $\overline{\cup_{j \geq 1} D_{j}}$ and $F_{2}-F_{2}^{\prime}$ on $\left(\overline{\cup_{j \geq 1} D_{j}}\right)^{c}$ is harmonic and has limit zero at infinity. Thus $H=0$. This proves the uniqueness part of the theorem.

\section{Proofs of results of Subsection 5.1}

Proof of Proposition 5.2. Let $V=\left(V_{\beta}\right)_{\beta \in B}$ be an open cover of $S$. Consider the open cover $W=\left(W_{\gamma}\right)_{\gamma \in \Gamma}$ consisting of all possible sets $V_{\beta} \cap U_{\alpha}$ where $\left(U_{\alpha}, \varphi_{\alpha}\right) \in \mathcal{D}$ is a chart. Since $S$ is paracompact of covering dimension $\leq n$ having a base of relatively compact open subsets, there exist locally finite open refinements $W^{\prime}=$ $\left(W_{i}^{\prime}\right)_{i \in I}$ of $W$ and $W^{\prime \prime}=\left(W_{i}^{\prime \prime}\right)_{i \in I}$ of $W^{\prime}$ such that the order of $W^{\prime}$ is $\leq n+1$ and $W_{i}^{\prime \prime} \Subset W_{i}^{\prime}$ for each $i \in I$, see, e.g. arguments in the proof of Lemma 1.4 in [30].

To prove the result it suffices for each $i \in I$ to construct a nonnegative $C^{k}$ function $g_{i}$ on $S$ such that $g_{i}>0$ on $\bar{W}_{i}^{\prime \prime}$ and $\operatorname{supp} g_{i} \subset W_{i}^{\prime}$. Then

$$
\rho_{i}:=\frac{g_{i}}{\sum_{s \in I} g_{s}}, \quad i \in I,
$$

is the required $C^{k}$ partition of unity.

To this end for each $\bar{W}_{i}^{\prime \prime} \subset W_{i}^{\prime}$ choose $\left(U_{\alpha(i)}, \varphi_{\alpha(i)}\right) \in \mathcal{D}$ such that $W_{i}^{\prime} \subset U_{\alpha(i)}$. Then $\varphi_{\alpha(i)}\left(\bar{W}_{i}^{\prime \prime}\right)$ is a compact subset of the relatively open subset $\varphi_{\alpha(i)}\left(W_{i}^{\prime}\right)$ of a weak $k$-Markov set $S_{\alpha(i)}$ of $\mathbb{R}^{n}$. In a standard way we construct a nonnegative $C^{k}$ function $\tilde{g}_{i}$ on $\mathbb{R}^{n}$ positive on $\varphi_{\alpha(i)}\left(\bar{W}_{i}^{\prime \prime}\right)$ such that $\left(\operatorname{supp} \tilde{g}_{i}\right) \cap S_{\alpha(i)} \Subset \varphi_{\alpha(i)}\left(W_{i}^{\prime}\right)$, see, e.g., [30]. Finally, we determine $g_{i}:=\varphi_{\alpha(i)}^{*} \tilde{g}_{i}$.

Proof of Proposition 5.3. By definitions of the objects involved, it suffices to prove the following version of the proposition:

If $S_{1}, S_{2} \in \operatorname{Mar}_{k}^{*}\left(\mathbb{R}^{n}\right), k \geq 1$, and $f: S_{1} \rightarrow S_{2}$ is a $C^{k}$ diffeomorphism, then $f^{-1}: S_{2} \rightarrow S_{1}$ is a $C^{k}$ diffeomorphism as well.

In this case the definition of the $C^{k}$ diffeomorphism and the (classical) inverse function theorem in $\mathbb{R}^{n}$ imply that there exist open neighbourhoods $U_{1}$ of $S_{1}$ and $U_{2}$ of $S_{2}$ and a $C^{k}$ extension $\hat{f}: U_{1} \rightarrow U_{2}$ of $f$ which is a $C^{k}$ diffeomorphism. Therefore $\hat{f}^{-1}: U_{2} \rightarrow U_{1}$ is a $C^{k}$ diffeomorphism of manifolds, see, e.g., [30]. Hence, $f^{-1}:=\left.\hat{f}^{-1}\right|_{S_{2}}: S_{2} \rightarrow S_{1}$ is a $C^{k}$ diffeomorphism by definition.

Proof of Proposition 5.4. Since the covering dimension of $S$ is at most $n$ and $S$ is separable, it can be covered by at most $n+1$ charts $\left(U_{i}, \varphi_{i}\right)_{1 \leq i \leq m}$ so that each $U_{i}$ is disjoint union of at most countable family of mutually disjoint open sets 
$V_{i j} \Subset S$ such that $\varphi_{i}\left(V_{i j}\right) \Subset \mathbb{R}^{n}$ is of diameter (measured in the Euclidean norm) at most $1 / 4$. Choose vectors $v_{i j} \in \mathbb{R}^{n}$ such that $v_{i j}+\varphi_{i}\left(V_{i j}\right)$ contain $0 \in \mathbb{R}^{n}$. Let $L \subset \mathbb{R}^{n}$ be a one-dimensional subspace containing a unit vector $e$. Choose a sequence of points $\left\{x_{i j}\right\} \subset L$ enumerated by the same indices as the family $\left\{V_{i j}\right\}$ such that $x_{i j}:=n_{i j} e, n_{i j} \in \mathbb{N}$. In particular, distances between distinct points of the sequence are greater than or equal to 1 .

Let $\left\{\rho_{i j}\right\}$ be a $C^{k}$ partition of unity subordinate to the open cover $\left(V_{i j}\right)$ of $S$. Then $\rho_{i}:=\sum_{j} \rho_{i j}, 1 \leq i \leq m$, is a $C^{k}$ partition of unity subordinate to the open cover $\left(U_{i}\right)$. We define maps $\tilde{\varphi}_{i}: S \rightarrow \mathbb{R}^{n}$,

$$
\tilde{\varphi}_{i}(x):=\rho_{i}(x)\left(x_{i}(x)+v_{i}(x)+\varphi_{i}(x)\right), \quad x \in S .
$$

Here $x_{i}:=\sum_{j} x_{i j} \chi_{i j}$ and $v_{i}:=\sum_{j} v_{i j} \chi_{i j}$, where $\chi_{i j}$ is the characteristic function of $V_{i j}$.

Then we define a map $\Phi: S \rightarrow \mathbb{R}^{m(n+1)}$ by the formula

$$
\Phi(x):=\left(\tilde{\varphi}_{1}(x), \ldots, \tilde{\varphi}_{m}(x), \rho_{1}(x), \ldots, \rho_{m}(x)\right) .
$$

Note that $\Phi(x)=\Phi\left(x^{\prime}\right)$ implies that $\rho_{i}(x)=\rho_{i}\left(x^{\prime}\right) \neq 0$ for some $i$ and hence $x, x^{\prime} \in U_{i}$. Assume that $x \in V_{i j}$ and $x^{\prime} \in V_{i j^{\prime}}$. Then $\rho_{i j}(x)=: \rho_{i}(x)=\rho_{i}\left(x^{\prime}\right):=$ $\rho_{i j^{\prime}}\left(x^{\prime}\right)$. In turn, this implies that $x_{i j}+v_{i j}+\varphi_{i}(x)=x_{i j^{\prime}}+v_{i j^{\prime}}+\varphi_{i}\left(x^{\prime}\right)$. Hence, if $j \neq j^{\prime}$, then $1 \leq\left\|x_{i j}-x_{i j^{\prime}}\right\|_{2}=\left\|\left(v_{i j^{\prime}}+\varphi_{i}\left(x^{\prime}\right)\right)-\left(v_{i j}+\varphi_{i}(x)\right)\right\|_{2} \leq 1 / 2$, a contradiction. Thus $j=j^{\prime}$ and $\varphi_{i}(x)=\varphi_{i}\left(x^{\prime}\right)$, i.e., $x=x^{\prime}$.

Now we check that $D \Phi$ is injective on each fibre of $T S$. At each point $x \in S$ the differential $D \Phi(x)$ sends a vector $v \in T_{x} S \subset T S$ of the tangent space at $x$ to the following vector in $\mathbb{R}^{n} \times \cdots \times \mathbb{R}^{n} \times \mathbb{R} \times \cdots \times \mathbb{R} \subset T_{\Phi(x)} \mathbb{R}^{m(n+1)}$ :

$$
\begin{aligned}
(D \Phi(x))(v):= & \left(\left(D \rho_{1}(x)\right)(v)\left(x_{1}(x)+v_{1}(x)+\varphi_{1}(x)\right)+\rho_{1}(x)\left(D \varphi_{1}(x)\right)(v), \ldots,\right. \\
& \left(D \rho_{m}(x)\right)(v)\left(x_{m}(x)+v_{m}(x)+\varphi_{m}(x)\right)+\rho_{m}(x)\left(D \varphi_{m}(x)\right)(v), \\
& \left.\left(D \rho_{1}(x)\right)(v), \ldots,\left(D \rho_{m}(x)\right)(v)\right) .
\end{aligned}
$$

Let $1 \leq s \leq m$ be such that $\rho_{s}(x) \neq 0$. Assuming that $(D \Phi(x))(v)=0$ we get $\left(D \varphi_{s}(x)\right)(v)=0$, a contradiction.

Finally, let us show that $\Phi$ is proper, i.e., preimage under $\Phi$ of a compact subset of $\mathbb{R}^{m(n+1)}$ is compact in $S$. Assuming, on the contrary, that this is false we find a sequence $\left\{s_{n}\right\}_{n \in \mathbb{N}} \subset S$ without limit points such that $\left\{\Phi\left(s_{n}\right)\right\}_{n \geq 1} \subset \mathbb{R}^{m(n+1)}$ is bounded. This implies that the sequence $\left\{\sum_{i=1}^{m} \rho_{i}\left(s_{n}\right) x_{i}\left(s_{n}\right)\right\}_{n>1} \subset L$ is bounded. We get from here that $\sup _{n>1} \min _{i \in J_{n}}\left\|x_{i}\left(s_{n}\right)\right\|_{2}<\infty$, where $J_{n}$ is the set of indices $i \in\{1, \ldots, m\}$ for which $\rho_{i}\left(s_{n}\right) \neq 0$. Since $\left\|x_{i}\left(s_{n}\right)\right\|_{2} \in \mathbb{N}$ for all $i \in J_{n}$ and $n \in \mathbb{N}$, the latter inequality implies that there exist a number $i_{0}$ and an infinite subsequence $\left\{s_{n_{p}}\right\}_{p \in \mathbb{N}}$ of $\left\{s_{n}\right\}$ such that $x_{i_{0}}\left(s_{n_{p}}\right) \neq 0$ and are equal for all $p \in \mathbb{N}$. This is possible only if all $s_{n_{p}}$ belong to some $V_{i_{0} j_{0}}$ contradicting our assumption (because $V_{i_{0} j_{0}} \subset S$ is relatively compact but $\left\{s_{n_{p}}\right\}$ does not have limit points in $S$ ). Thus $\Phi$ is proper.

Lemma 9.1. Let $S \in \operatorname{Mar}_{k}^{*}, k \geq 1$, and the rank of $T S$ be $n$. Suppose $f: S \rightarrow M$ is a $C^{k}$ map into a smooth Riemannian manifold $M$ of $\operatorname{dim} M>n$. Then $f(S)$ has measure zero. 
Proof. Since $S$ can be covered by at most countable family of charts it suffices to prove the result for $S \in \operatorname{Mar}_{k}^{*}\left(\mathbb{R}^{n}\right)$. Next, since $f$ is $C^{k}$ and $M$ is a smooth manifold, there is an open neighbourhood $U \subset \mathbb{R}^{n}$ of $S$ and a $C^{k}$ map $\hat{f}: U \rightarrow M$ such that $\left.\hat{f}\right|_{S}=f$. Then by the classical Sard theorem $\hat{f}(U)$ is of measure zero in $M$. Since $f(S) \subset \hat{f}(U)$ is union of a countable family of compact subsets, it is measurable and so has measure zero as well.

If $S \in \operatorname{Mar}_{k}^{*}$ with $k \geq 2$, starting with the proper embedding $\Phi: S \rightarrow \mathbb{R}^{N}$, $N:=m(n+1)>2 n+1$, as above, we then show that by projecting onto a hyperplane it is possible to obtain an embedding into $\mathbb{R}^{N-1}$.

A vector $v \in \mathbb{S}^{N-1} \subset \mathbb{R}^{N}$ defines a hyperplane (the orthogonal complement) and let $P_{v}: \mathbb{R}^{N} \rightarrow \mathbb{R}^{N-1}$ be the orthogonal projection onto this hyperplane. We show that the set of $v$ for which $\Phi_{v}=P_{v} \circ \Phi$ fails to be a proper embedding is of measure zero, hence that it is possible to choose $v$ for which $\Phi_{v}$ is a proper embedding.

The map $\Phi_{v}$ fails to be an embedding exactly when it is not injective or $D \Phi_{v}$ is not injective at some point. Let us consider the two failures separately:

If $v$ is in the image of the map $\beta:(S \times S) \backslash \Delta_{S} \rightarrow \mathbb{S}^{n-1}$, where $\Delta_{S} \subset S \times S$ is the diagonal, given by

$$
\beta\left(x_{1}, x_{2}\right):=\frac{\Phi\left(x_{1}\right)-\Phi\left(x_{2}\right)}{\left\|\Phi\left(x_{1}\right)-\Phi\left(x_{2}\right)\right\|_{2}},
$$

then $\Phi_{v}$ will fail to be injective. Note however that $\beta$ maps a space in $\operatorname{Mar}_{k}^{*}, k \geq 2$, with rank of tangent bundle $2 n$ into $(N-1)$-dimensional Riemannian manifold, and if $N>2 n+1$, then Lemma 9.1 implies that the image has measure zero.

The immersion condition is a local one, which we may analyze in a chart $(U, \varphi)$. The map $\Phi_{v}$ will fail to be an immersion in $U$ precisely when $v$ coincides with a vector in the normalized image of $D\left(\Phi \circ \varphi^{-1}\right)$, where

$$
\Phi \circ \varphi^{-1}: \varphi(U) \subset \mathbb{R}^{n} \longrightarrow \mathbb{R}^{N} .
$$

Hence, we have a map (letting $N(w)=\|w\|_{2}$ )

$$
\frac{D\left(\Phi \circ \varphi^{-1}\right)}{N \circ D\left(\Phi \circ \varphi^{-1}\right)}: \varphi(U) \times \mathbb{S}^{n-1} \longrightarrow \mathbb{S}^{N-1} .
$$

Note that since $k \geq 2$, this map is at least of class $C^{1}$. Thus, by Lemma 9.1, its image has measure zero as long as $2 n-1<N-1$, which is certainly true since $2 n<N-1$. Taking union over at most countably many charts, we see that immersion fails on a set of measure zero in $\mathbb{S}^{N-1}$.

In addition, $\Phi_{v}$ is not proper if there exists a sequence $\left\{s_{n}\right\} \subset S$ such that $\left\{\Phi\left(s_{n}\right)\right\} \subset \mathbb{R}^{N}$ converges to $\infty$ and $\left\{\Phi_{v}\left(s_{n}\right)\right\} \subset \mathbb{R}^{N-1}$ is bounded. This implies that limit points of $\left\{\Phi\left(s_{n}\right) /\left\|\Phi\left(s_{n}\right)\right\|_{2}\right\}$ are either $v$ or $-v$. However, by the definition of $\Phi$ limit points of the latter sequence coincide with limit points of the sequence

$$
\begin{aligned}
& \left\{\frac{\left(\rho_{1}\left(s_{n}\right) x_{1}\left(s_{n}\right), \ldots, \rho_{m}\left(s_{n}\right) x_{m}\left(s_{n}\right), 0, \ldots, 0\right)}{\left.\| \rho_{1}\left(s_{n}\right) x_{1}\left(s_{n}\right), \ldots, \rho_{m}\left(s_{n}\right) x_{m}\left(s_{n}\right), 0, \ldots, 0\right) \|_{2}}\right\}_{n \geq 1} \\
& \subset \mathbb{S}^{N-1} \cap(L \times \cdots \times L \times\{0\} \times \cdots \times\{0\})=: M \cong \mathbb{S}^{m-1} .
\end{aligned}
$$


Since $m-1 \leq n<N-1, M$ is a set of measure zero in $\mathbb{S}^{N-1}$. Thus for $\Phi_{v}$ to be proper it suffice to choose $v \notin M$.

We see that $\Phi_{v}$ fails to be a proper embedding for a set of $v \in \mathbb{S}^{N-1}$ of measure zero. Hence repeating the above arguments (with $\Phi$ replaced by a suitable $\Phi_{v}$ ) we may reduce $N$ all the way to $N=2 n+1$.

Next, let us show that if $f: S \rightarrow \mathbb{R}^{N}$ is a proper embedding, then $f(S) \in \operatorname{Mar}_{k}^{*}$ and has weak $k$-Markov structure induced from $\mathbb{R}^{N}$.

Let $(U, \varphi)$ be a chart on $S$. By definition the map $f \circ \varphi^{-1}: \varphi(U) \subset \mathbb{R}^{n} \rightarrow \mathbb{R}^{N}$ is a $C^{k}$ embedding. In particular, there exist an open neighbourhood $O \subset \mathbb{R}^{n}$ of $\varphi(U)$ and a $C^{k}$ embedding $\hat{f}: O \rightarrow \mathbb{R}^{N}$ which coincides with $f \circ \varphi^{-1}$ on $\varphi(U)$. Since $f$ is a proper embedding, $f(U) \subset f(S)$ is open in the induced from $\mathbb{R}^{N}$ topology and for each point $x \in \varphi(U)$ there exists an open ball $B_{x} \Subset O$ centered at $x$ such that $\hat{f}\left(B_{x}\right) \cap f(S)=\left(f \circ \varphi^{-1}\right)\left(B_{x} \cap \varphi(U)\right)$. Then $\hat{f}\left(B_{x}\right)$ is a $C^{k}$ submanifold of the $C^{k}$ manifold

$$
M_{x}:=\mathbb{R}^{N} \backslash\left(\hat{f}\left(\partial B_{x}\right) \cup\left(f(S) \backslash\left(f \circ \varphi^{-1}\right)\left(B_{x} \cap \varphi(U)\right)\right)\right)
$$

here $\partial B_{x}$ is the boundary of $B_{x}$. By the tubular neighbourhood theorem, there exists an open neighbourhood $N_{x} \subset M_{x}$ of $\hat{f}\left(B_{x}\right)$ and a $C^{k}$ retraction $r_{x}: N_{x} \rightarrow$ $\hat{f}\left(B_{x}\right)$. In particular, $N_{x} \cap f(S)=\left(f \circ \varphi^{-1}\right)\left(B_{x} \cap \varphi(U)\right)$.

By the definition $\left.\varphi \circ f^{-1}\right|_{\left(f \circ \varphi^{-1}\right)\left(B_{x} \cap \varphi(U)\right)}$ is restriction to $f(U)$ of the $C^{k}$ map $\hat{f}^{-1} \circ r_{x}: N_{x} \rightarrow \mathbb{R}^{n}$. Using the open cover $\left(N_{x}\right)_{x \in \varphi(U)}$ of $f(U)$ we find its locally finite open refinement $\left(W_{i}\right)_{i \in I}$ and a subordinate $C^{\infty}$ partition of unity $\left\{\rho_{i}\right\}_{i \in I}$ with supp $\rho_{i} \Subset W_{i}$. If $\tau: I \rightarrow \varphi(U)$ is a refinement map such that $W_{i} \subset N_{\tau(i)}$, then we define the map $g: W:=\cup_{i \in I} W_{i} \rightarrow \mathbb{R}^{n}$ by the formula

$$
g(x):=\sum_{i \in I} \rho_{i}(x) \cdot\left(\hat{f}^{-1} \circ r_{\tau(i)}\right)(x), \quad x \in W .
$$

For $x \in \hat{f}(\varphi(U))$, let $i_{1}, \ldots, i_{q}$ be all indices for which $\rho_{i_{m}}(x) \neq 0,1 \leq m \leq q$. Then

$$
x \in\left(\bigcap_{m=1}^{q} N_{\tau\left(i_{m}\right)}\right) \bigcap f(S)=\bigcap_{m=1}^{q}\left(f \circ \varphi^{-1}\right)\left(B_{\tau\left(i_{m}\right)} \cap \varphi(U)\right),
$$

so that

$$
g(x)=\sum_{m=1}^{q} \rho_{i_{m}}(x)\left(\varphi \circ f^{-1}\right)(x)=\left(\varphi \circ f^{-1}\right)(x) .
$$

Hence,

$$
\left.g\right|_{f(U)}=\varphi \circ f^{-1}
$$

Finally, we equip $f(S)$ with the weak $k$-Markov structure consisting of all charts of the form $\left(f(U), \varphi \circ f^{-1}\right)$. Since $f$ is proper, $f(U)$ is open in the induced topology so that the above argument shows that $f(S) \in \operatorname{Mar}_{k}^{*}$ and has the weak $k$-Markov structure induced from $\mathbb{R}^{N}$ and $f: S \rightarrow f(S)$ is a $C^{k}$ diffeomorphism. 


\section{Proof of Theorem 5.5}

\subsection{Hausdorff dimension of graphs of continuous functions}

In this subsection we formulate and prove auxiliary results used in the proof of the theorem.

In what follows $\operatorname{dim}_{H}$ and $\underline{\operatorname{dim}}_{B}$ and $\overline{\operatorname{dim}}_{B}$ will denote the Hausdorff and lower and upper box-counting dimensions of a bounded subset of a Euclidean space. If $\underline{\operatorname{dim}}_{B}(F)=\overline{\operatorname{dim}}_{B}(F)$ we refer to this value as the box-counting dimension of $F$ (denoted $\operatorname{dim}_{B}(F)$ ). We will use the following product formulas, see, e.g., page 94 in $[10]$ :

If $E \subset \mathbb{R}^{n}$ and $F \subset \mathbb{R}^{m}$ are any Borel sets, then

$$
\operatorname{dim}_{H} E+\operatorname{dim}_{H} F \leq \operatorname{dim}_{H}(E \times F) \leq \operatorname{dim}_{H} E+\overline{\operatorname{dim}}_{B}(F) .
$$

Let $f: I \subset \mathbb{R} \rightarrow \mathbb{R}$ be bounded continuous on an interval $I$ and $\Gamma_{f} \subset \mathbb{R}^{2}$ be its graph. In many cases $\Gamma_{f}$ may be fractal. We formulate several results of this type (for other results, see, e.g., [9], [10], [21], [31], [32] and references therein).

If

$$
w_{\Theta}(x):=\sum_{n=0}^{\infty} a^{n} \cos \left(2 \pi\left(b^{n} x+\theta_{n}\right)\right), \quad 0<a<1<b, \quad a b>1,
$$

is the Weierstrass function with a random phase added to each term, and if each $\theta_{n}$ is chosen independently with respect to the uniform probability measure in $[0,1]$, then with probability one the Hausdorff dimension of the graph of $w_{\Theta}$ over each (nontrivial) subinterval $I \subset \mathbb{R}$ is $D:=2+(\ln a) /(\ln b)$, see Theorem 1 and pages 796-798 in [21]. In the case of all $\theta_{n}=0$, i.e., of the classical Weierstrass function $w$, it remains still unknown whether the Hausdorff dimension of $\Gamma_{w}$ has the same value although it is proved in [23] that $\operatorname{dim}_{B} \Gamma_{w}=D$.

Also, it was shown on page 796 of [21] that on each nontrivial subinterval $I \subset \mathbb{R}$, the function $w_{\Theta}$ satisfies the Hölder condition with exponent $2-D$. Thus according to Corollary 11.2 of [10], for almost all sequences $\Theta=\left\{\theta_{n}\right\}$ chosen independently with respect to the uniform probability measure in $[0,1]$, the graph of $w_{\Theta}$ over each (nontrivial) subinterval $I \subset \mathbb{R}$ satisfies

$$
\operatorname{dim}_{H}\left(\left.\Gamma_{w_{\Theta}}\right|_{I}\right)=\overline{\operatorname{dim}}_{B}\left(\left.\Gamma_{w_{\Theta}}\right|_{I}\right)=D
$$

Now, if $\Gamma_{w_{\Theta_{1}}}, \ldots, \Gamma_{w_{\Theta_{n}}} \subset \mathbb{R}^{2}$ are graphs of functions satisfying (10.3), then according to the product formula (10.1)

$$
\operatorname{dim}_{H}\left(\left.\Gamma_{w_{\Theta_{1}}}\right|_{I_{1}} \times \cdots \times\left.\Gamma_{w_{\Theta}}\right|_{I_{n}} \times \overline{\mathbb{K}}^{s}\right)=n D+s
$$

for all nontrivial closed intervals $I_{1}, \ldots, I_{n} \subset \mathbb{R}$.

Observe that $D$ attains any value in open interval $(1,2)$. Thus $n D$ attains any value in the interval $(n+s, 2 n+s)$. Moreover, $\left.\Gamma_{w_{\Theta_{1}}}\right|_{I_{1}} \times \cdots \times\left.\Gamma_{w_{\Theta_{n}}}\right|_{I_{n}} \times \overline{\mathbb{K}}^{s} \in$ $\operatorname{Mar}_{\infty}^{*}\left(\mathbb{R}^{2 n+s}\right)\left(\overline{\mathbb{K}}^{s}:=[0,1]^{s}\right)$ for all nontrivial closed intervals $I_{1}, \ldots, I_{n} \subset \mathbb{R}$, see properties (5) and (8) of Section 2. 
Example 10.1. The graph of the Weierstrass function

$$
f(x)=\sum_{n=1}^{\infty}\left(\frac{7}{10}\right)^{n} \cos \left(9^{n} \pi x\right)
$$

has a base of (induced) topology of sets of Hausdorff dimension more than one, see, e.g., [10] and [32].

Further, it was proved in [40] by relatively simple methods that for the function

$$
\Phi(x):=\sum_{k=1}^{\infty} \lambda^{-k^{\alpha}} \phi\left(\lambda^{k^{\beta}} x\right), \quad x \in[0,1],
$$

where $\lambda>1, \beta>\alpha>1$, and $\phi(x)=2 x$ for $0 \leq x \leq 1 / 2, \phi(-x)=\phi(x)$ and $\phi(x+1)=\phi(x)$, its graph over any nontrivial closed subinterval of $[0,1]$ has Hausdorff dimension 2. Taking the direct product of $n$ copies of graphs of such functions and of $\overline{\mathbb{K}}^{s}$ we obtain the graph of a continuous map $\overline{\mathbb{K}}^{n+s} \rightarrow \overline{\mathbb{K}}^{n}$ having Hausdorff dimension $2 n+s$ over each nondegenerate closed cube in $\overline{\mathbb{K}}^{n+s}$ (cf. (10.1)). Also, this graph belongs to $\operatorname{Mar}_{\infty}^{*}\left(\mathbb{R}^{2 n+s}\right)$ due to properties (5) and (8) of Section 2.

Finally, in the proofs of Theorem 6.6 and Proposition 6.10 we constructed bounded Lipschitz functions $\mathbb{R} \rightarrow \mathbb{R}$ whose graphs either belong to $\operatorname{Mar}_{k}^{*}\left(\mathbb{R}^{2}\right) \backslash$ $\operatorname{Mar}_{k+1}^{*}\left(\mathbb{R}^{2}\right)$ for a fixed $k \in \mathbb{N}$ or to $\operatorname{Mar}_{\infty}^{*}\left(\mathbb{R}^{2}\right)$. In turn, taking direct products of $n$ copies of such graphs with $\mathbb{R}^{s}$ we obtain graphs of Lipschitz maps $\mathbb{R}^{n+s} \rightarrow \mathbb{R}^{n}$ either belonging to $\operatorname{Mar}_{k}^{*}\left(\mathbb{R}^{2 n+s}\right) \backslash \operatorname{Mar}_{k+1}^{*}\left(\mathbb{R}^{2 n+s}\right)$ for a fixed $k \in \mathbb{N}$ or to $\operatorname{Mar}_{\infty}^{*}\left(\mathbb{R}^{2 n+s}\right)$, see property (5) of Section 2.

In the proof of the theorem we use the following result.

Suppose $\Gamma_{F}:=\{(x, F(x)) ; x \in Q\} \subset \mathbb{R}^{2 n}$ is the graph of a continuous map $F: Q \subset \mathbb{R}^{n} \rightarrow \mathbb{R}^{n}$ defined over a closed nondegenerate cube $Q$. Assume that

(P) $\Gamma_{F}$ is either belongs to $\operatorname{Mar}_{k}^{*}\left(\mathbb{R}^{2 n}\right) \backslash \operatorname{Mar}_{k+1}^{*}\left(\mathbb{R}^{2 n}\right)$ for a fixed $k \in \mathbb{N}$, or to $\operatorname{Mar}_{\infty}^{*}\left(\mathbb{R}^{2 n}\right)$, or has a base of (induced) topology of sets of Hausdorff dimension $n \leq d \leq 2 n$.

Let $T \Subset Q$ be a convex open subsets and let $\rho$ be a $C^{\infty}$ function on $\mathbb{R}^{n}$ with all derivatives equal to zero on $T^{c}:=\mathbb{R}^{n} \backslash T$ and nonzero at each point of $T$ (e.g., as such a $\rho$ one may take a regularized distance to the complement of $T$, see [35]).

Lemma 10.2. The graph $\Gamma_{\rho F}:=\{(x, \rho(x) F(x)) ; x \in \bar{T}\}$ of $\rho F$ over $\bar{T}$ has the same properties as $\Gamma_{F}$, see $(\mathrm{P})$.

Proof. Let us define a $C^{\infty}$ map $\tilde{\rho}: \mathbb{R}^{2 n} \rightarrow \mathbb{R}^{2 n}$ by the formula $\tilde{\rho}(x, y):=(x, \rho(x) y)$, $(x, y) \in \mathbb{R}^{n} \times \mathbb{R}^{n}=\mathbb{R}^{2 n}$. Then by the hypothesis $\tilde{\rho}$ has a $C^{\infty}$ inverse on $T \times \mathbb{R}^{n}$. Now implications $\Gamma_{\rho F} \in \operatorname{Mar}_{k}^{*}\left(\mathbb{R}^{2 n}\right) \backslash \operatorname{Mar}_{k+1}^{*}\left(\mathbb{R}^{2 n}\right)$ for a fixed $k \in \mathbb{N}$, or $\Gamma_{\rho F} \in$ $\operatorname{Mar}_{\infty}^{*}\left(\mathbb{R}^{2 n}\right)$ follow from property (6) of Section 2 provided that the same is true for $\Gamma_{F}$. The remaining property follows from the fact that $\tilde{\rho}$ is locally bi-Lipschitz over $T \times \mathbb{R}^{n}$ and so preserves Hausdorff dimension of any Borel measurable subset of this set. 


\subsection{Proof of Theorem 5.5}

Proof. According to the assumptions of the theorem, there exists an open neighbourhood $U^{\prime} \subset U$ of $M$ and a $C^{\infty}$ submanifold $M^{\prime}$ of $U^{\prime}$ such that $M$ and $M^{\prime}$ are $C^{k}$ isotopic inside $U^{\prime}$, see, e.g., Section 4 in [30]. Proving the result for $M^{\prime}$ and taking suitable compositions of the isotopies between $M_{r}^{\prime}$ and $M^{\prime}, r \in\{d, l\}$, and $M^{\prime}$ and $M$, we obtain the required result for $M$. Thus without loss of generality we may assume in the proof that $M$ is of class $C^{\infty}$.

Let $N_{M} \rightarrow M$ be the normal bundle on $M$. There exists a convex open neighbourhood $W$ of the zero section $M \hookrightarrow N_{M}$ and a $C^{\infty}$ diffeomorphism $g: W \rightarrow X$ whose image $N$ is a tubular neighbourhood of $M$ in $U$, see, e.g., Section 5 in [30]. Thus it suffices to construct the required objects in $W$ and then embed them into $X$ by $g$.

To this end recall that since $M$ is of class $C^{\infty}$, it admits a $C^{\infty}$ triangulation (see, e.g., Section 10 in [30]). Shrinking this triangulation, if necessary, we may assume that $N_{M}$ is $C^{\infty}$ trivial in an open neighbourhood of the closure of each simplex of the triangulation. Now each nondegenerate simplex $\sigma$ of the triangulation is $C^{\infty}$ diffeomorphic to the standard $m$-dimensional simplex $\Delta \subset \overline{\mathbb{K}}^{m}$. Thus we may identify (by means of $C^{\infty}$ diffeomorphisms) $\sigma$ with $\Delta$ and $N_{M}$ on $\sigma$ with $\Delta \times \mathbb{R}^{n-m}$. Using results of the previous subsection we construct graphs $\Gamma_{\sigma} \subset \mathbb{R}^{n}$ of continuous maps $F_{\sigma}: \Delta \rightarrow \mathbb{R}^{n-m}$ equal zero on the boundary of $\Delta$ such that $\Gamma_{\sigma}$ either belong to $\operatorname{Mar}_{l}^{*}\left(\mathbb{R}^{n}\right) \backslash \operatorname{Mar}_{l+1}^{*}\left(\mathbb{R}^{n}\right)$ for a fixed $\ell \in \mathbb{N}$ (in this case the corresponding maps $F_{\sigma}$ are Lipschitz), or to $\operatorname{Mar}_{\infty}^{*}\left(\mathbb{R}^{n}\right)$ and have bases of (induced) topology of sets of Hausdorff dimension $m \leq d \leq n$ (in case $d=m$ maps $F_{\sigma}$ are Lipschitz as well). Using diffeomorphisms identifying $\sigma$ with $\Delta$ and $N_{M}$ on $\sigma$ with $\Delta \times \mathbb{R}^{n-m}$, we may regard each $F_{\sigma}$ as a continuous section of $N_{M}$ over $\sigma$ equals zero on the boundary of $\sigma$. Choosing $F_{\sigma}$ so that $\max _{\sigma}\left\|F_{\sigma}\right\|_{2}$ are sufficiently small, we may assume that images of all $F_{\sigma}$ belong to $W$. Let us define a continuous section $F: M \rightarrow W$ by the formula $\left.F\right|_{\sigma}:=F_{\sigma}$ for all $\sigma$ of the triangulation of $M$. We set $M_{d}:=(g \circ F)(M)$ if all $\Gamma_{\sigma}$ have bases of (induced) topology of sets of Hausdorff dimension $m \leq d \leq n$ and $M_{l}:=(g \circ F)(M)$ if all $\Gamma_{\sigma} \in \operatorname{Mar}_{l}^{*}\left(\mathbb{R}^{n}\right) \backslash \operatorname{Mar}_{l+1}^{*}\left(\mathbb{R}^{n}\right)$. Moreover, since $W$ is convex, $(1-t) F(M) \subset W$ for all $t \in[0,1]$. We define the required homotopy $h_{r, t}(x):=g\left((1-t) g^{-1}(x)\right), x \in M_{r}, t \in[0,1]$; here $r \in\{d, l\}$. It is a matter of definitions to check that the introduced objects satisfy the required conditions.

\section{Proof of Theorem 5.6}

Due to the definitions of $\operatorname{Mar}_{\infty, \Gamma}^{*}$ and of $\Omega^{p}(S)$ (see Subsection 5.2), it suffices to prove the following version of the theorem.

Theorem 11.1. Let $S=\Gamma_{f_{11}, f_{12}}^{n_{1}} \times \cdots \times \Gamma_{f_{\ell 1}, f_{\ell 2}}^{n_{\ell}} \Subset \mathbb{R}^{n}:=\mathbb{R}^{n_{1}+1} \times \cdots \times \mathbb{R}^{n_{\ell}+1}$ be $a \Gamma$ set such that $\bar{S} \in \operatorname{Mar}_{\infty}^{*}\left(\mathbb{R}^{n}\right)$. Let $\omega:=\sum_{|I|=p} c_{I} d x_{I}$ be a d-closed $C^{\infty} p$-form on $\bar{S}$. Then for $p \geq 1$ and an open subset $U \Subset S$ there exists a $C^{\infty}(p-1)$-form $\eta_{U}$ on $U$ such that $d \eta_{U}=\left.\omega\right|_{U}$. If $p=0$, then $\omega$ is a constant function on $S$. 
Proof. We will prove the result by induction on $\ell$. For $\ell=1$ we prove the following more general statement.

Let $S_{1} \times S_{2} \Subset \mathbb{R}^{n+1} \times \mathbb{R}^{m}$ be such that $S_{1} \Subset \mathbb{R}^{n+1}, \bar{S}_{1} \in \operatorname{Mar}_{\infty}^{*}\left(\mathbb{R}^{n+1}\right)$, is a simple $\Gamma$ set and $S_{2} \Subset \mathbb{R}^{m}, \bar{S}_{2} \in \operatorname{Mar}_{\infty}^{*}\left(\mathbb{R}^{m}\right)$, is a $\Gamma$ set. Let $x=\left(x_{1}, \ldots, x_{n+1}\right)$ and $y=\left(y_{1}, \ldots, y_{m}\right)$ be the standard coordinates on $\mathbb{R}^{n+1}$ and $\mathbb{R}^{m}$, respectively. By $d_{x}$ we denote the differential on $\Omega^{*}\left(\bar{S}_{1} \times \bar{S}_{2}\right)$ with respect to the coordinate $x$ for $y$ fixed. For a differential form $\omega=\sum c_{I J} d x_{I} \wedge d y_{J}$ on $\bar{S}_{1} \times \bar{S}_{2}$ we set $\operatorname{deg}_{x} \omega:=$ $\max \left\{|I| ; c_{I J} \not \equiv 0\right\}$.

Lemma 11.2. Suppose that $\omega$ is a $d_{x}$-closed $C^{\infty}$ differential form on $\bar{S}_{1} \times \bar{S}_{2}$. If $\operatorname{deg}_{x} \omega \geq 1$, then there exists a $C^{\infty}$ differential form $\eta$ on $S_{1} \times \bar{S}_{2}$ such that $d_{x} \eta=\left.\omega\right|_{S_{1} \times \bar{S}_{2}}$. If $\operatorname{deg}_{x} \omega=0$, then $\omega$ is independent of $x$.

Proof of Lemma 11.2. Without loss of generality we may assume that

$$
S_{1}=\left\{\left(x_{1}, \ldots, x_{n}, t f_{1}\left(x_{1}, \ldots, x_{n}\right)+(1-t) f_{2}\left(x_{1}, \ldots, x_{n}\right)\right) ; x \in \mathbb{K}^{n}, t \in[0,1]\right\}
$$

for some continuous $f_{1}, f_{2}: \mathbb{K}^{n} \rightarrow \mathbb{R}, \mathbb{K}^{n}:=(0,1)^{n}$, such that $f_{1} \leq f_{2}$. By the definition of $C^{\infty}$ functions on weak $\infty$-Markov sets (see Section 4 ), $\omega$ is restriction to $\bar{S}_{1} \times \bar{S}_{2}$ of a $C^{\infty}$ differential form $\omega^{\prime}$ (of the same degree as $\omega$ ) defined in an open neighbourhood of $\bar{S}_{1} \times \bar{S}_{2}$ in $\mathbb{R}^{n+1} \times \mathbb{R}^{m}$. Since $\bar{S}_{1} \times \bar{S}_{2}$ is compact, multiplying $\omega^{\prime}$ by a suitable $C^{\infty}$ cut-off function, we obtain a $C^{\infty}$ differential form $\widetilde{\omega}$ on $\mathbb{R}^{n+1} \times \mathbb{R}^{m}$ such that $\left.\widetilde{\omega}\right|_{\bar{S}_{1} \times \bar{S}_{2}}=\omega$ and $\operatorname{deg} \widetilde{\omega}=\operatorname{deg} \omega, \operatorname{deg}_{x} \widetilde{\omega}=\operatorname{deg}_{x} \omega$.

Since $d_{x} \widetilde{\omega}=0$ on $\bar{S}_{1} \times \bar{S}_{2}$ and $\bar{S}_{1} \in \operatorname{Mar}_{\infty}^{*}\left(\mathbb{R}^{n+1}\right)$,

$$
d_{x}\left(\left.\widetilde{\omega}\right|_{\left(\mathbb{K}^{n} \times \mathbb{R}\right) \times \bar{S}_{2}}\right)=\beta_{-}+\beta_{+},
$$

where each $\beta_{-}(\cdot, y)$ coincides with $d_{x} \widetilde{\omega}(\cdot, y)$ on the connected component of $\left(\left(\mathbb{K}^{n} \times \mathbb{R}\right) \backslash S_{1}\right) \times\{y\}$ containing $\mathbb{K}^{n} \times\{-\infty\} \times\{y\}$ and on the other connected component of $\left(\left(\mathbb{K}^{n} \times \mathbb{R}\right) \backslash S_{1}\right) \times\{y\}$ equals zero. Similarly, each $\beta_{+}(\cdot, y)$ coincides with $d_{x} \widetilde{\omega}(\cdot, y)$ on the connected component of $\left(\left(\mathbb{K}^{n} \times \mathbb{R}\right) \backslash S_{1}\right) \times\{y\}$ containing $\mathbb{K}^{n} \times\{+\infty\} \times\{y\}$ and on the other connected component of $\left(\left(\mathbb{K}^{n} \times \mathbb{R}\right) \backslash S_{1}\right) \times\{y\}$ equals zero; here $y \in \bar{S}_{2}$ (see Section 8 for similar arguments). By the definition of the $d_{x}$ operator on weak $\infty$-Markov sets, the differential forms $\beta_{ \pm}$are of class $C^{\infty}$ on $\left(\mathbb{K}^{n} \times \mathbb{R}\right) \times \bar{S}_{2}$. We write $\beta_{ \pm}=\left(\widetilde{\omega}_{n+1}\right)_{ \pm}+\lambda_{ \pm}$, where $\left(\widetilde{\omega}_{n+1}\right)_{ \pm}:=d x_{n+1} \wedge \tilde{\lambda}_{ \pm}$ and $\lambda_{ \pm}$do not contain $d x_{n+1}$. Since $d_{x} \omega=0$, the forms $\beta_{ \pm}$are $d_{x}$-closed on $\left(\mathbb{K}^{n} \times \mathbb{R}\right) \times \bar{S}_{2}$.

We set

$$
\theta_{ \pm}(x, y):=\int_{ \pm \infty}^{x_{n+1}} \tilde{\lambda}_{ \pm}\left(x_{1}, \ldots, x_{n}, t_{n+1}, y\right) d t_{n+1} .
$$

Clearly, $\theta_{ \pm}$are of class $C^{\infty}$ on $\left(\mathbb{K}^{n} \times \mathbb{R}\right) \times \bar{S}_{2}$. Now, $\beta_{ \pm}-d_{x} \theta_{ \pm}$are $d_{x}$-closed $C^{\infty}$ differential forms on $\left(\mathbb{K}^{n} \times \mathbb{R}\right) \times \bar{S}_{2}$ not containing terms with $d x_{n+1}$. Hence, partial derivatives of their coefficients with respect to $x_{n+1}$ are zeros. In particular, these coefficients are zeros, because at each $y \in \bar{S}_{2}$ the forms $\left(\beta_{ \pm}-d_{x} \theta_{ \pm}\right)(\cdot, y)$ are equal to zero on the corresponding connected components containing $\mathbb{K}^{n} \times\{ \pm \infty\} \times\{y\}$.

Thus we have

$$
\beta_{ \pm}=\left(\widetilde{\omega}_{n+1}\right)_{ \pm}=d_{x} \theta_{ \pm}
$$


In turn, equality $d_{x}\left(\left.\widetilde{\omega}\right|_{\left(\mathbb{K}^{n} \times \mathbb{R}\right) \times \bar{S}_{2}}\right)=\beta_{+}+\beta_{-}=d_{x}\left(\theta_{+}+\theta_{-}\right)$and the classical Poincaré lemma (for $d$-closed differential forms on $\mathbb{K}^{n} \times \mathbb{R}$ ) imply that if $\operatorname{deg}_{x} \widetilde{\omega}=$ $\operatorname{deg}_{x} \omega \geq 1$, then there exists a $C^{\infty}$ differential form $\eta$ on $\left(\mathbb{K}^{n} \times \mathbb{R}\right) \times \bar{S}_{2}$ such that $\left.\widetilde{\omega}\right|_{\left(\mathbb{K}^{n} \times \mathbb{R}\right) \times \bar{S}_{2}}-\theta_{+}-\theta_{-}=d_{x} \eta$. Moreover, by our construction forms $\theta_{ \pm}$equal zero on $S_{1} \times \bar{S}_{2}$ so that the previous equality implies that

$$
d_{x}\left(\left.\eta\right|_{S_{1} \times \bar{S}_{2}}\right)=\omega
$$

completing the proof of the lemma in this case.

Next, if $\operatorname{deg}_{x} \widetilde{\omega}=\operatorname{deg}_{x} \omega=0$, then $\left.\widetilde{\omega}\right|_{\left(\mathbb{K}^{n} \times \mathbb{R}\right) \times \bar{S}_{2}}-\theta_{+}-\theta_{-}=\sum c_{J} d y_{J}$ and $d_{x} c_{J}=0$ for all $J$ on $\left(\mathbb{K}^{n} \times \mathbb{R}\right) \times \bar{S}_{2}$. Hence, all $c_{J}$ do not depend on $x \in \mathbb{K}^{n} \times \mathbb{R}$, i.e., $\left.\widetilde{\omega}\right|_{\left(\mathbb{K}^{n} \times \mathbb{R}\right) \times \bar{S}_{2}}-\theta_{+}-\theta_{-}$is a form in $y$. Since $\theta_{ \pm}$equal zero on $S_{1} \times \bar{S}_{2}$, the differential form $\omega:=\left.\widetilde{\omega}\right|_{\bar{S}_{1} \times \bar{S}_{2}}$ depends on $y$ only.

Lemma 11.2 shows that the theorem is valid for $\ell=1$ and any open $U \Subset S$. Assuming that it is valid for $\ell-1$ let us establish it for $\ell$.

To this end by $x=\left(x_{1}, \ldots, x_{n_{\ell}+1}\right)$ and $y:=\left(y_{1}, \ldots, y_{n-n_{\ell}-1}\right)$ we denote the standard coordinates on $\mathbb{R}^{n_{\ell}+1}$ and $\mathbb{R}^{n-n_{\ell}-1}:=\mathbb{R}^{n_{1}+1} \times \cdots \times \mathbb{R}^{n_{\ell-1}+1}$, respectively. We will prove the statement by induction on $\operatorname{deg}_{x} \omega$.

For $\operatorname{deg}_{x} \omega=0$ from $d$-closedness of $\omega$ and Lemma 11.2 we obtain that $\omega$ is a $d$-closed form depending on $y$ only. Thus it is pullback to $S$ (under the natural projection $\mathbb{R}^{n} \rightarrow \mathbb{R}^{n_{\ell-1}+1}$ ) of a $d$-closed differential form on $\bar{\Gamma}_{f_{11}, f_{12}}^{n_{1}} \times \cdots \times$ $\bar{\Gamma}_{f_{\ell-11}, f_{\ell-12}}^{n_{\ell-1}} \Subset \mathbb{R}^{n-n_{\ell-1}}$ and, since the number of terms in the direct product is $\ell-1$, the required statement (of the theorem) follows from the first induction hypothesis.

Suppose that we have proved the statement for $\operatorname{deg}_{x} \omega:=s-1 \geq 0$; let us prove it for $\operatorname{deg}_{x} \omega:=s$.

We write $\omega=\omega_{1}+\omega_{2}$, where $\omega_{1}$ is the sum of all terms of $\omega$ of $\operatorname{deg}_{x} \omega:=s$ and $\operatorname{deg}_{x} \omega_{2} \leq s-1$. Then $d$-closedness of $\omega$ implies that $d_{x} \omega_{1}=0$. Therefore from Lemma 11.2 we obtain that $\omega_{1}=d_{x} \eta_{1}$ for some $C^{\infty}$ differential form on $S$ with $\operatorname{deg}_{x} \eta_{1} \leq s-1$. Consider the form $\omega-d \eta_{1}:=\omega_{2}-d_{y} \eta_{1}$. It is $d$-closed on $S$ with $\operatorname{deg}_{x}$ at most $s-1$. Thus by the induction hypotheses applied to a $\Gamma$ set $U^{\prime} \Subset S$ containing $\bar{U},\left.\omega\right|_{U^{\prime}}=d \eta_{U^{\prime}}$ for a $C^{\infty}$ differential form $\eta_{U^{\prime}}$ on $U^{\prime}$. This proves the step of the second induction and therefore the step of the first induction completing the proof of the theorem.

\section{Proofs of Corollary 5.11 and Theorems 5.12 and 5.13}

\subsection{Proof of Corollary 5.11}

Lemma 12.1. Any set of the form

$$
Z:=\left\{\left(x, t f_{1}(x)+(1-t) f_{2}(x)\right) \in \mathbb{R}^{n+1} ; x \in \overline{\mathbb{K}}^{n}, t \in[0,1]\right\}
$$

for some continuous $f_{1}, f_{2}: \overline{\mathbb{K}}^{n} \rightarrow \mathbb{R}$ such that $f_{1} \leq f_{2}$, is a strong deformation retract of $\mathbb{R}^{n+1}$. 
Proof of Lemma 12.1. We refer to [20] for basic definitions of homotopy theory.

Clearly $\overline{\mathbb{K}}^{n} \times \mathbb{R}$ is a strong deformation retract of $\mathbb{R}^{n+1}$. Thus it suffices to check that $Z$ is a strong deformation retract of $\overline{\mathbb{K}}^{n} \times \mathbb{R}$. For $\left(x, x_{n+1}, s\right) \in\left(\overline{\mathbb{K}}^{n} \times \mathbb{R}\right) \times[0,1]$ we define the required retraction $F:\left(\overline{\mathbb{K}}^{n} \times \mathbb{R}\right) \times[0,1] \rightarrow Z$ as follows:

$$
F\left(x, x_{n+1}, s\right):=\left\{\begin{array}{rll}
\left(x, f_{2}(x)+s\left(x_{n+1}-f_{2}(x)\right)\right) & \text { if } & x_{n+1} \geq f_{2}(x) \\
\left(x, x_{n+1}\right) & \text { if } & f_{1}(x) \leq x_{n+1} \leq f_{2}(x), \\
\left(x, f_{1}(x)+s\left(x_{n+1}-f_{1}(x)\right)\right) & \text { if } & x_{n+1} \leq f_{1}(x) .
\end{array}\right.
$$

The lemma implies, in particular, that finite direct products of sets of the form (12.1) are absolute retracts. Also, any $S \in \operatorname{Mar}_{\infty, \Gamma}^{*}$ is countable union of such finite direct products, see Subsection 5.2; hence, by a result of Hanner [16], $S$ is an absolute neighbourhood retract. In particular, in the setting of the theorem, there exists an open neighbourhood $N \subset X$ of $S$ and a continuous retraction $r: N \rightarrow S$. We embed $X$ as a closed subset into some $\mathbb{R}^{n}$ by Proposition 5.4 so that the weak $\infty$-Markov structure on $X$ is induced from that on $\mathbb{R}^{n}$. Thus without loss of generality we may assume in the proof that $X$ (and therefore $N$ ) belongs to $\operatorname{Mar}_{\infty, \Gamma}^{*}$.

By $i: S \hookrightarrow N$ we denote the $\left(C^{\infty}\right)$ embedding map. Then the pullbacks of $r$ and $i$ define the following homomorphisms of Cech cohomology groups:

$$
r_{C}^{*}: \check{H}^{p}(S, \mathbb{R}) \longrightarrow \check{H}^{p}(N, \mathbb{R}), \quad i_{C}^{*}: \check{H}^{p}(N, \mathbb{R}) \longrightarrow \check{H}^{p}(S, \mathbb{R})
$$

such that $i_{C}^{*} \circ r_{C}^{*}=\mathrm{id}$. Thus

$$
\check{H}^{p}(N, \mathbb{R})=r_{C}^{*}\left(\check{H}^{p}(S, \mathbb{R})\right) \oplus \operatorname{Ker} i_{C}^{*} .
$$

For a $d$-closed form $\omega \in \Omega^{p}(S)$, we denote by $[\omega] \in H_{d R}^{p}(S, \mathbb{R})$ its de Rham cohomology class. Consider

$$
\left(r_{C}^{*} \circ\left(h_{S}^{p}\right)^{-1}\right)([\omega]) \in \check{H}^{p}(N, \mathbb{R}),
$$

where $h_{S}^{p}: \check{H}^{p}(S, \mathbb{R}) \rightarrow H_{d R}^{p}(S, \mathbb{R})$ is the isomorphism of Theorem 5.8 for $S$. Since $N \in \operatorname{Mar}_{\infty, \Gamma}^{*}$, the de Rham theorem is valid on $N$, i.e., $h_{N}^{p}: \check{H}^{p}(N, \mathbb{R}) \rightarrow$ $H_{d R}^{p}(N, \mathbb{R})$ is an isomorphism (see (5.1)). Let $\widehat{\omega} \in \Omega^{p}(N)$ be a $d$-closed form whose de Rham cohomology class coincides with $\left(h_{N}^{p} \circ r_{C}^{*} \circ\left(h_{S}^{p}\right)^{-1}\right)([\omega])$. Using the commutative diagram (5.1) for the map $i$ and identity $i_{C}^{*} \circ r_{C}^{*}=$ id we obtain

$$
\left(i_{d R}^{*} \circ h_{N}^{p} \circ r_{C}^{*} \circ\left(h_{S}^{p}\right)^{-1}\right)([\omega])=\left(h_{S}^{p} \circ i_{C}^{*} \circ r_{C}^{*} \circ\left(h_{S}^{p}\right)^{-1}\right)([\omega])=[\omega] .
$$

Hence, the restriction of $\widehat{\omega}$ to $S$ belongs to the same de Rham cohomology class as $\omega$. Therefore $\omega-\left.\widehat{\omega}\right|_{S}=d \eta$ with $\eta \in \Omega^{p-1}(S)$ (here we assume that $p \geq 1$, for otherwise, the statement of the theorem is trivial). As $\eta$ admits locally $C^{\infty}$ extensions to open subsets of $N$, using a suitable open cover of the closed set $S$ and a subordinate to it $C^{\infty}$ partition of unity, we patch such extensions together to find a $C^{\infty}(p-1)$-form $\widehat{\eta}$ on $N$ such that $\left.\widehat{\eta}\right|_{S}=\eta$. We set $\widetilde{\omega}:=\widehat{\omega}-d \widehat{\eta}$. Then $\left.\widetilde{\omega}\right|_{S}=\omega$. 


\subsection{Proof of Theorem $\mathbf{5 . 1 2}$}

Assume first that $S \in \operatorname{Mar}_{\infty, \Gamma}^{*}$ is a closed subset of some $\mathbb{R}^{n}$ with the induced weak $\infty$-Markov structure ( $S$ admits such an embedding by Proposition 5.4 ). By $i: S \hookrightarrow \mathbb{R}^{n}$ we denote the $C^{\infty}$ embedding and by $r: N \rightarrow S$ a continuous retraction of an open neighbourhood $N \subset \mathbb{R}^{n}$ of $S$ onto $S$, cf. Theorem 5.11. Then the induced homomorphisms

$$
r_{*}: H_{k}(N, \mathbb{R}) \rightarrow H_{k}(S, \mathbb{R}) \quad \text { and } \quad i_{*}: H_{k}(S, \mathbb{R}) \rightarrow H_{k}(N, \mathbb{R})
$$

of singular homology groups satisfy $r_{*} \circ i_{*}=\mathrm{id}$. In particular, $i_{*}$ is an injection and $H_{k}(N, \mathbb{R})=i_{*}\left(H_{k}(S, \mathbb{R})\right) \oplus \operatorname{Ker} r_{*}$.

Let $\omega$ be a $d$-closed $k$-form on $S$ and $[\omega] \in H_{d R}^{k}(S, \mathbb{R})$ be its cohomology class. We choose a $d$-closed $k$-form $\widetilde{\omega}$ on $N$ such that $\left.\widetilde{\omega}\right|_{S}=\omega$ (existing by Theorem 5.11 ). Let $\sigma$ be a singular $k$-cycle in $S$ and let $[\sigma] \in H_{k}(S, \mathbb{R})$ stand for its homology class. We define a map $\Phi_{S}: H_{d R}^{k}(S, \mathbb{R}) \rightarrow H^{k}(S, \mathbb{R})$ by the formula

$$
\left\langle\Phi_{S}([\omega]),[\sigma]\right\rangle:=\int_{\sigma^{\prime}} \widetilde{\omega}, \quad[\sigma] \in H_{k}(S, \mathbb{R}),
$$

where $\sigma^{\prime}$ is a piecewise smooth singular chain in $N$ with homology class $i_{*}([\sigma])$.

Identifying $[\widetilde{\omega}] \in H_{d R}^{k}(N, \mathbb{R})$ with an element of $H^{k}(N, \mathbb{R})$ by the classical de Rham theorem, we obtain that the expression on the right in (12.2) can be written as $\left\langle i^{*}([\widetilde{\omega}]),[\sigma]\right\rangle$, where $i^{*}: H^{k}(N, \mathbb{R}) \rightarrow H^{k}(S, \mathbb{R})$ is the map transpose to $i_{*}$.

We must check that $\Phi_{S}$ is correctly defined, independent of embedding into $\mathbb{R}^{n}$ and determines the required isomorphism of cohomology rings.

To check that $\Phi_{S}$ is correctly defined, suppose that $\widetilde{\omega}^{\prime}$ is another $d$-closed $k$ form on $N$ satisfying $\left.\widetilde{\omega}^{\prime}\right|_{S}=\omega$. Using the isomorphism $h_{N}^{*}$ between Cech and de Rham cohomology on $N$ expressed in Theorem 5.8, consider $\left(h_{N}^{k}\right)^{-1}\left(\left[\widetilde{\omega}-\widetilde{\omega}^{\prime}\right]\right) \in \check{H}^{k}(N, \mathbb{R})$. Then due to $(5.1)$

$$
\left(i_{C}^{*} \circ\left(h_{N}^{k}\right)^{-1}\right)\left(\left[\widetilde{\omega}-\widetilde{\omega}^{\prime}\right]\right)=\left(\left(h_{S}^{k}\right)^{-1} \circ i_{d R}^{*}\right)\left(\left[\widetilde{\omega}-\widetilde{\omega}^{\prime}\right]\right)=\left(h_{S}^{k}\right)^{-1}(0)=0 .
$$

Let $K \subset S$ be compact such that $\sigma \in Z_{k}(K, \mathbb{R})$, the abelian group of singular cycles in $K$. Since $K$ is projective limit of the system of its open relatively compact neighbourhoods $U$ in $N$ with the partial order defined by inclusion, $\check{H}^{k}(K, \mathbb{R})$ is isomorphic to inductive $\operatorname{limit} \lim \check{H}^{k}(U, \mathbb{R})$ taking along this system (see, e.g., [1] for basic results of sheaf theory). Since $\omega-\widetilde{\omega}=0$ on $S$,

$$
\left(\left(i_{K \hookrightarrow U}\right)_{C}^{*} \circ\left(h_{U}^{k}\right)^{-1}\right)\left(\left[\left.\left(\widetilde{\omega}-\widetilde{\omega}^{\prime}\right)\right|_{U}\right]\right)=0,
$$

where $i_{K \hookrightarrow U}: K \rightarrow U$ is the embedding. As $\check{H}^{k}(K, \mathbb{R})$ is the inductive limit of groups $\breve{H}^{k}(U, \mathbb{R})$, the latter implies that there exists open $U_{0} \Subset N$ containing $K$ such that $\left(h_{U_{0}}^{k}\right)^{-1}\left(\left[\left.\left(\widetilde{\omega}-\widetilde{\omega}^{\prime}\right)\right|_{U_{0}}\right]\right)=0$ in $\check{H}^{k}\left(U_{0}, \mathbb{R}\right)$, that is $\left.\left(\widetilde{\omega}-\widetilde{\omega}^{\prime}\right)\right|_{U_{0}}$ is $d$-exact. Let $\sigma^{\prime} \in Z_{k}\left(U_{0}, \mathbb{R}\right)$ be a piecewise smooth singular $k$-cycle in $U_{0}$ homologous to $\sigma$. Then the $d$-exactness of $\left.\left(\widetilde{\omega}-\widetilde{\omega}^{\prime}\right)\right|_{U_{0}}$ implies that

$$
\int_{\sigma^{\prime}} \widetilde{\omega}=\int_{\sigma^{\prime}} \widetilde{\omega}^{\prime}
$$


Since (12.2) does not depend on the choice of $\sigma^{\prime} \in Z_{k}(N, \mathbb{R})$, the above equality shows that (12.2) does not depend on the choice of the extension $\widetilde{\omega}$ of $\omega$; similarly it is independent of the choice of the form representing the class $[\omega]$ as well. Thus $\Phi_{S}$ is correctly defined.

Next, if $\Phi_{S}([\omega])=0$, then $[\widetilde{\omega}]_{s} \in \operatorname{Ker} i^{*}$ (here $[\widetilde{\omega}]_{s}$ stands for the singular cohomology class determined by $\widetilde{\omega}$ as above via the de Rham theorem on $N$ ). Since $S$ (being an absolute neighbourhood retract) is homotopy equivalent to a $C W$ complex, see, e.g., Theorem 1 in [28], singular cohomology on $S$ is naturally isomorphic to Čech cohomology. Thus

$$
\left.\left(h_{N}^{k}\right)^{-1}\right)([\widetilde{\omega}]) \in \operatorname{Ker} i_{C}^{*} \subset \check{H}^{k}(N, \mathbb{R}) \cong H_{d R}(N, \mathbb{R}) .
$$

Therefore,

$$
0=\left(i_{C}^{*} \circ\left(h_{N}^{k}\right)^{-1}\right)([\widetilde{\omega}])=\left(\left(h_{S}^{k}\right)^{-1} \circ i_{d R}^{*}\right)([\widetilde{\omega}])=\left(h_{S}^{k}\right)^{-1}([\omega]) .
$$

Thus, by Theorem $5.8,[\omega]=0 \in H_{d R}^{k}(S, \mathbb{R})$ showing that $\Phi_{S}$ is an injection.

Further, for $\lambda \in H^{k}(S, \mathbb{R})$ consider $r^{*}(\lambda) \in H^{k}(N, \mathbb{R})$. Let $[\widetilde{\omega}] \in H_{d R}^{k}(N, \mathbb{R})$ be the de Rham cohomology class representing $r^{*}(\lambda)$ (existing by the de Rham theorem for $C^{\infty}$ manifolds). Since $\left(i^{*} \circ r^{*}\right)(\lambda)=\lambda$, by $(12.2)$ we have $\Phi_{S}([\omega])=$ $i^{*}([\widetilde{\omega}])=\lambda$ for $\omega:=\left.\widetilde{\omega}\right|_{S}$. This shows that $\Phi_{S}$ is a surjection.

The fact that $\Phi_{S}\left(\lambda_{1} \wedge \lambda_{2}\right)=\Phi_{S}\left(\lambda_{1}\right) \smile \Phi_{S}\left(\lambda_{2}\right), \lambda_{1}, \lambda_{2} \in H_{d R}^{*}(S, \mathbb{R})$, follows from (12.2) and the similar identity for cohomology on $N$ (valid by the classical de Rham theorem for $C^{\infty}$ manifolds).

Thus $\Phi_{S}: H_{d R}^{*}(S, \mathbb{R}) \rightarrow H^{*}(S, \mathbb{R})$ is a ring isomorphism.

Next, we show that $\Phi_{S}$ does not depend on embedding $i: S \hookrightarrow \mathbb{R}^{n}$.

Suppose that $i_{p}: S \hookrightarrow \mathbb{R}^{n_{p}}, p=1,2$, are proper $C^{\infty}$ embeddings. Consider the proper $C^{\infty}$ embedding $i:=\left(i_{1}, i_{2}\right): S \hookrightarrow \mathbb{R}^{n}:=\mathbb{R}^{n_{1}} \times \mathbb{R}^{n_{2}}$. By $\pi_{p}: \mathbb{R}^{n} \rightarrow \mathbb{R}^{n_{p}}$, $p=1,2$, we denote the natural projections. Clearly, $\pi_{p} \circ i=i_{p}, p=1,2$. Due to the preceding discussion, the map $\Phi_{i(S)}: H_{d R}^{*}(i(S), \mathbb{R}) \rightarrow H^{*}(i(S), \mathbb{R})$ is well defined. Moreover, due to $(12.2)$ for $[\omega] \in H_{d R}^{k}(S, \mathbb{R})$ and $\sigma \in H_{k}(S, \mathbb{R})$ we have

$$
\begin{aligned}
\left\langle\left( i_{p}^{*} \circ \Phi_{i_{p}(S)} \circ\right.\right. & \left.\left.\left(i_{p}^{*}\right)^{-1}\right)([\omega]), \sigma\right\rangle \\
& =\left\langle\left(\Phi_{i_{p}(S)} \circ\left(\left(\left.\pi_{p}\right|_{i(S)}\right)^{*}\right)^{-1} \circ\left(i^{*}\right)^{-1}\right)([\omega]),\left(\pi_{p} \circ i\right)_{*}(\sigma)\right\rangle \\
& =\left\langle\left(\Phi_{i(S)} \circ\left(i^{*}\right)^{-1}\right)([\omega]),\left(\left(\left.\pi_{p}\right|_{i(S)}\right)^{-1} \circ \pi_{p} \circ i\right)_{*}(\sigma)\right\rangle \\
& =\left\langle\left(\Phi_{i(S)} \circ\left(i^{*}\right)^{-1}\right)([\omega]),(i)_{*}(\sigma)\right\rangle .
\end{aligned}
$$

Hence, $i_{p}^{*} \circ \Phi_{i_{p}(S)} \circ\left(i_{p}^{*}\right)^{-1}=i^{*} \circ \Phi_{i(S)} \circ\left(i^{*}\right)^{-1}, p=1,2$. This shows that $\Phi_{S}$ does not depend on embedding into $\mathbb{R}^{n}$.

Let us prove now the commutativity of diagram (5.2). So assume that $f: S_{1} \rightarrow S_{2}$ is a $C^{\infty}$ map of spaces in $\operatorname{Mar}_{\infty, \Gamma}^{*}$. Without loss of generality we may assume that $S_{i} \subset \mathbb{R}^{n_{i}}, i=1,2$, are closed and equipped with the induced weak $\infty$-Markov structures. We embed $S_{1}$ into $S_{1} \times S_{2}$ as the closed subset $\Gamma_{f}:=\left\{\left(s_{1}, f\left(s_{1}\right)\right) ; s_{1} \in S_{1}\right\}$ and by $\pi_{2}: \mathbb{R}^{n_{1}} \times \mathbb{R}^{n_{2}} \rightarrow \mathbb{R}^{n_{2}}$ we denote the natural projection. Thus we may assume that $S_{1} \subset \mathbb{R}^{n_{1}} \times \mathbb{R}^{n_{2}}$ (equipped with the induced weak $\infty$-Markov structure, 
see arguments in the proof of Proposition 5.4) and $f:=\left.\pi_{2}\right|_{S_{1}}$. Since $S_{2} \subset \mathbb{R}^{n_{2}}$, by definition (12.2) for $[\omega] \in H_{d R}^{k}\left(S_{2}, \mathbb{R}\right)$ and $\sigma \in H_{k}\left(S_{1}, \mathbb{R}\right)$ we have

$$
\left\langle\left(f^{*} \circ \Phi_{S_{2}}\right)([\omega]), \lambda\right\rangle=\left\langle\Phi_{S_{2}}([\omega]), f_{*}(\lambda)\right\rangle=\left\langle\left(\Phi_{S_{1}} \circ f_{d R}^{*}\right)([\omega]), \lambda\right\rangle,
$$

as required.

The statement asserting that in the case of $S$ being a $C^{\infty}$ manifold $\Phi_{S}$ coincides with the classical de Rham isomorphism follows from the fact that for $S \subset \mathbb{R}^{n}$ being a $C^{\infty}$ submanifold the singular chain $\sigma^{\prime}$ in $(12.2)$ can be chosen from $Z^{k}(S, \mathbb{R})$.

The proof of the theorem is complete.

\subsection{Proof of Theorem $\mathbf{5 . 1 3}$}

We define a map $\times: H^{*}\left(S_{1}, \mathbb{R}\right) \otimes H^{*}\left(S_{2}, \mathbb{R}\right) \rightarrow H^{*}\left(S_{1} \times S_{2}, \mathbb{R}\right)$ of singular cohomology rings by the formula

$$
\lambda_{1} \times \lambda_{2}:=\pi_{1}^{*}\left(\lambda_{1}\right) \smile \pi_{2}^{*}\left(\lambda_{2}\right), \quad \lambda_{1} \otimes \lambda_{2} \in H^{*}\left(S_{1}, \mathbb{R}\right) \otimes H^{*}\left(S_{2}, \mathbb{R}\right) .
$$

Due to Theorem 5.12 we have

$$
\Phi_{S_{1} \times S_{2}}\left(c_{1} \wedge c_{2}\right)=\Phi_{S_{1}}\left(c_{1}\right) \times \Phi_{S_{2}}\left(c_{2}\right), \quad c_{1} \otimes c_{2} \in H_{d R}^{*}\left(S_{1}, \mathbb{R}\right) \otimes H_{d R}^{*}\left(S_{2}, \mathbb{R}\right) .
$$

In turn, it is known, see, e.g., Appendix A in [29], that as spaces $S_{1}$ and $S_{2}$ are homotopy equivalent to $C W$ complexes, under the assumptions of the theorem,

$$
\times: H^{*}\left(S_{1}, \mathbb{R}\right) \otimes H^{*}\left(S_{2}, \mathbb{R}\right) \rightarrow H^{*}\left(S_{1} \times S_{2}, \mathbb{R}\right)
$$

is an isomorphism. Since $\Phi_{S_{1} \times S_{2}}, \Phi_{S_{1}}$ and $\Phi_{S_{2}}$ are ring isomorphisms, this fact and the previous identity imply the required.

\section{References}

[1] Bredon, G. E.: Sheaf theory. Graduate Texts in Mathematics 170, Springer-Verlag, New York, 1997.

[2] Brudnyi, A. And Brudnyi, Yu.: Methods of geometric analysis in extension and trace problems, Volumes 1 and 2. Mathematical Monographs 102 and 103, Birkhäuser/Springer, Basel, 2012.

[3] Brudnyi, A. and Brudnyi, Yu.: Traces of functions of the class $C^{k}$ on weakly Markov subsets of $\mathbb{R}^{n}$. St. Petersburg Math. J. 23 (2012), no. 1, 39-56.

[4] Brudnyi, Yu. and Ganzburg, M.: A certain extremal problems for polynomials in $n$ variables. Izv. Akad. Nauk SSSR 37 (1973), 344-355; English translation in: Math. USSR-Izv. 7 (1973), 345-356.

[5] Bierstone, E. And Milman, P. D.: Geometric and differential properties of subanalytic sets. Ann. of Math. (2) 147 (1998), no. 3, 731-785.

[6] Bierstone, E. And Milman, P. D.: $C^{m}$-norms on finite sets and $C^{m}$ extension criteria. Duke Math. J. 137 (2007), no. 1, 1-18. 
[7] Bos, L. And Milman, P. D.: Sobolev-Gagliardo-Nirenberg and Markov type inequalities on subanalytic domains. Geom. Funct. Anal. 5 (1995), no. 6, 853-923.

[8] Bott, R. And Tu, L. W.: Differential forms in algebraic topology. Graduate Texts in Mathematics 82, Springer-Verlag, New York-Berlin, 1982.

[9] Besicovitch, A.S. And Ursell, H. D.: Sets of fractional dimensions (V): on dimensional numbers of some continuous curves. J. London Math. Soc. (2) 32 (1937), $142-153$.

[10] FALCONER, K.: Fractal geometry: mathematical foundations and applications. John Wiley, New York, 1990.

[11] Fefferman, C.: A generalized sharp Whitney theorem for jets. Rev. Mat. Iberoamericana 21 (2005), no. 2, 577-688.

[12] Fefferman, C.: Interpolation and extrapolation of smooth functions by linear operators. Rev. Mat. Iberoamericana 21 (2005), no. 1, 313-348.

[13] Fefferman, C.: Whitney's extension problems and interpolation of data. Bull. Amer. Math. Soc. (N.S.) 46 (2009), no. 2, 207-220.

[14] Gilbarg, D. And Trudinger, N.: Elliptic partial differential equations of second order. Grundlehren der Mathematischen Wissenschaften 224, Springer-Verlag, Berlin, 1983.

[15] Hardy, G. H.: Weierstrass's nondifferentiable function. Trans. Amer. Math. Soc. 17 (1916), no. 3, 301-325.

[16] Hanner, O.: Some theorems on absolute neighborhood retracts. Ark. Mat. 1 (1951), 389-408.

[17] Henkin, G.: The method of integral representations in complex analysis. In Current problems in mathematics. Fundamental directions, Vol. 7, 23-124. Itogi Nauki i Tekhniki. Akad. Nauk SSSR, Vsesoyuz. Inst. Nauchn. i Tekn. Inform., Moscow 1985.

[18] Hestens, M.: Extension of the range of a differentiable function. Duke Math. J. 8 (1941), 183-192.

[19] Hirzebruch, F.: Topological methods in algebraic geometry. Die Grundlehren der Mathematischen Wissenschaften 131, Springer-Verlag, New York, 1966.

[20] Hu, S. T.: Homotopy theory. Pure and Applied Mathematics 8, Academic Press, New York-London, 1959.

[21] Hunt, B. R.: The Hausdorff dimensions of graphs of Weierstrass functions. Proc. Amer. Math. Soc. 126 (1998), no. 3, 791-800.

[22] Jonsson, A. And Wallin, H.: Function spaces on subsets of $\mathbb{R}^{n}$. Math. Rep. 2 (1984), no. 1, 1-221.

[23] Kaplan, J. L., Mallet-Paret, J. And Yorke, J. A.: The Lyapunov dimension of a nowhere differentiable attracting torus. Ergodic Theory Dynam. Systems 4 (1984), no. 2, 261-281.

[24] Kirby, R. C. And Siebenmann, L. C.: On the triangulation of manifolds and the Hauptvermutung. Bull. Amer. Math. Soc. 75, (1969), 742-749.

[25] Levin, B. JA.: Distribution of zeros of entire functions. American Mathematical Society, Providence, RI, 1964.

[26] Luli, G. K.: $C^{m, \omega}$ extension by bounded-depth linear operators. Adv. Math. 224 (2010), no. 5, 1927-2021.

[27] McShane, E. J.: Extension of range of function. Bull. Amer. Math. Soc. 40 (1934), no. $12,837-842$. 
[28] Milnor, J. W.: On spaces having the homotopy type of a CW-complex. Trans. Amer. Math. Soc. 90 (1959), 272-280.

[29] Milnor, J. W. And Stasheff, J. D.: Characteristic classes. Annals of Mathematics Studies 76, Princeton University Press, Princeton, NJ, 1974.

[30] Munkres, J. R.: Elementary differential topology. Annals of Mathematics Studies 54, Princeton University Press, Princeton, NJ, 1963.

[31] Mauldin, R. D. And Williams, S. C.: On the Hausdorff dimension of some graphs. Trans. Amer. Math. Soc. 298 (1986), no. 2, 793-803.

[32] Przytycki, F. And Urbanski, M.: On the Hausdorff dimension of some fractal sets. Studia Math. 93 (1989), no. 2, 155-186.

[33] Strichartz, R. S.: Analysis on fractals. Notices Amer. Math. Soc. 46 (1999), no. 10 , 1199-1208.

[34] Shvartsman, P.: The Whitney extension problem and Lipschitz selections of set-valued mappings in jet-spaces. Trans. Amer. Math. Soc. 360 (2008), no. 10, $5529-5550$

[35] Stein, E. M.: Singular integrals and differentiability properties of functions. Princeton Mathematical Series 30, Princeton University Press, Princeton, NJ, 1970.

[36] Whitney, H.: Analytic extension of differentiable functions defined in closed sets. Trans. Amer. Math. Soc. 36 (1934), no. 1, 63-89.

[37] Whitney, H.: Differentiable functions defined in closed sets. I. Trans. Amer. Math. Soc. 36 (1934), no. 2, 369-387.

[38] Whitney, H.: Differentiable manifolds. Ann. of Math. (2) 37 (1936), no. 3, 645-680.

[39] Whitney, H.: Geometric integration theory. Princeton University Press, Princeton, NJ, 1957.

[40] Xie, T.F. And Zhou, S. P.: On a class of fractal functions with graph Hausdorff dimension 2. Chaos Solitons Fractals 32 (2007), no. 5, 1625-1630.

Received May 3, 2013; revised June 11, 2013.

Alexander Brudnyi: Department of Mathematics and Statistics, University of Calgary, T2N 1N4 Calgary, Canada.

E-mail: albru@math.ucalgary.ca

Research is supported in part by NSERC. This is an extended version of my talk presented at 4th workshop on Whitney problems (College of William \& Mary, August 3-8, 2011). 